\title{
Physical principles and state-of-the-art of modeling of the pulsating heat pipe: A review
}

\author{
Vadim S. Nikolayev \\ Service de Physique de l'Etat Condensé, CEA, CNRS, Université Paris-Saclay, CEA Saclay, 91191 Gif-sur-Yvette Cedex, France
}

\begin{abstract}
The Pulsating (called also oscillating) Heat Pipe (PHP) is a high performance passive heat transfer device. It consists in a closed capillary channel folded into several meanders, evacuated, and partially filled with a liquid and its vapor. One side is in thermal contact with a hot spot, the other with a cold spot. The oscillation of the liquid plugs and vapor bubbles spontaneously occurs after the heating beginning. The heat exchange takes place not only by latent heat transfer, but also by liquid convection. Both this advantage and the PHP structural simplicity make it competitive with respect to other kinds of heat pipes. However, the PHP operation is non-stationary and depends on many physical and material parameters. As a result, application of empirical correlations is quite unsuccessful. More sophisticated direct modeling is thus required. In this review, we present the past, present, and future perspectives of the theoretical PHP studies. The physical phenomena on the level of a single bubble and single liquid plug is addressed first. In this context, the modeling of the simplest, single branch PHP is described as a necessary first step toward the PHP understanding and model validation. The modeling of interaction between the bubbles and plugs (bubble generation and disappearance, plug evaporation) is discussed next. Finally, the state of the art of the physical modeling and simulation of the multi-turn PHP is reviewed and the difference between existing approaches is explained.
\end{abstract}

Keywords: Pulsating heat pipe, Oscillating fluid flow, Theoretical modeling, Film evaporation, Taylor bubble

\section{Contents}

1 Introduction to the PHP predictive modeling

2 Physical phenomena and modeling of the PHP components

2.1 Flow and heat exchange in the liquid domain . 4

2.1.1 Pressure drop in the liquid plugs . . . . 4

2.1.2 Non stationary and non-adiabatic Taylor bubble flow .......... . 6

2.1.3 Heat transfer modeling in PHP . . . . . 6

2.2 Physics and modeling of vapor phase . . . . . . 7

2.2.1 Vapor thermodynamic state . . . . . . 7

2.2.2 Vapor energy equation ....... . 9

2.3 Liquid film dynamics in PHP . . . . . . . . . . . 9

2.3.1 Deposition of liquid films . . . . . 10

2.3.2 Film dynamics at oscillations .... 11

2.3.3 Film length: contact line dynamics . . . 12

2.3.4 Liquid film profile at phase change . . . 13

2.3.5 Contact line under phase change . . . . 14

2.3.6 Film modeling in existing PHP simulations ............. 16

2.4 Interaction between bubble or plug pairs . . . . 18

3 1D modeling of the single branch PHP 19

3.1 "Superheated vapor" modeling . . . . . . . . 20

3.2 FEC modeling . . . . . . . . . . . . . . 21
3.2.1 PHP startup model for imposed temperatures in evaporator and condenser . . . 21 3.2.2 Impact of the thermal fluid-solid coupling on the PHP startup . . . . . . 22

3.3 Experimental validation of the single branch PHP models . . . . . . . . . . . . . . 24

4 1D modeling of the multi-branch PHP 24

4.1 "Superheated vapor" modeling . . . . . . . . . 24

4.2 FEC approach for multi-branch PHP . . . . . . 25

4.3 Models with varying film thickness . . . . . . . 28

$52 D$ and 3D PHP simulations 28

6 Conclusions $\quad 30$ 


\section{Nomenclature}

$A$ meniscus oscillation amplitude [m]

$C$ viscous friction coefficient

$c$ specific heat $[\mathrm{J} /(\mathrm{kg} \cdot \mathrm{K})]$

$\mathrm{Ca}$ capillary number

$D$ heat diffusivity $\left[\mathrm{m}^{2} / \mathrm{s}\right]$

$d$ diameter of the PHP channel [m]

$e$ Euler number $\simeq 2.71 \ldots$

$F$ friction force [N]

$G$ gravity force [N]

$g$ gravity acceleration $\left[\mathrm{m}^{2} / \mathrm{s}\right]$

$H$ Hamaker constant [J]

$h$ specific enthalpy $[\mathrm{J} / \mathrm{kg}]$

$i$ imaginary unit $=\sqrt{-1}$

$I_{n}$ modified Bessel function of the order $n$

$J$ evaporation mass flux $\left[\mathrm{kg} /\left(\mathrm{m}^{2} \cdot \mathrm{s}\right)\right]$

$K$ interface curvature in the axial plane $\left[\mathrm{m}^{-1}\right]$

$L$ length [m]

$l, \ell$ characteristic length scales [m]

$\mathcal{L}$ latent heat $[\mathrm{J} / \mathrm{kg}]$

$M$ total number of bubbles or plugs

$m$ mass [kg]

$N$ total number

$\mathrm{Nu}$ Nusselt number

$P$ power [W], oscillation period in Fig. 10 [s]

$p$ pressure $[\mathrm{Pa}]$

$\wp$ perimeter of the PHP channel [m]

$\operatorname{Pr}$ Prandtl number

$Q$ heat amount [J]

$q$ heat flux $\left[\mathrm{W} / \mathrm{m}^{2}\right]$

$r$ radius of the PHP channel [m]

$R^{i}$ interfacial thermal resistance, $\left[\left(\mathrm{K} \cdot \mathrm{m}^{2}\right) / \mathrm{W}\right]$

$R_{v}$ gas constant $[\mathrm{J} /(\mathrm{kg} \cdot \mathrm{K})]$

Re Reynolds number

$S$ surface area $\left[\mathrm{m}^{2}\right]$

$T$ temperature [K]

$t$ time [s]

$U$ heat transfer coefficient $\left[\mathrm{W} /\left(\mathrm{m}^{2} \mathrm{~K}\right)\right]$

$u$ local fluid velocity $[\mathrm{m} / \mathrm{s}]$

$V$ liquid velocity averaged over cross-section $[\mathrm{m} / \mathrm{s}]$

$W$ characteristic macroscopic length [m]

$w$ dewetting ridge width [m]

$W e$ Weber number

$x, X, y, z$ coordinates [m]

\section{Abbreviations}

CASCO Code Avancé de Simulation de Caloduc Oscillant:

Advanced PHP simulation code in French

CFD computational fluid dynamics

CL contact line

EOS equation of state

FEC Film Evaporation-Condensation (model)
FR filling ratio of PHP by the liquid

PHP pulsating heat pipe

TC thermocouple

VOF volume of fluid (numerical method)

Greek symbols

$\gamma$ vapor adiabatic index $=c_{p v} / c_{v v}$

$\Delta$ difference

$\delta$ thickness [m]

$\theta$ interfacial slope, contact angle

$\lambda$ heat conductivity $[\mathrm{W} /(\mathrm{m} \cdot \mathrm{K})]$

$\mu$ liquid shear viscosity [Pa.s]

$v$ liquid kinematic viscosity $\left[\mathrm{m}^{2} / \mathrm{s}\right]$

$\Pi$ disjoining pressure $[\mathrm{Pa}]$

$\rho$ density $\left[\mathrm{kg} / \mathrm{m}^{3}\right]$

$\varrho$ radial coordinate $[\mathrm{m}]$

$\sigma$ surface tension $[\mathrm{N} / \mathrm{m}]$

$\varphi$ turn angle

$\Omega$ vapor bubble volume $\left[\mathrm{m}^{3}\right]$

$\omega$ angular frequency $\left[\mathrm{s}^{-1}\right]$

Superscripts

$i$ interfacial

$l$ left

$r$ right

$s r$ or $l$

\section{Subscripts}

$a$ adiabatic section

app apparent

$b$ branch

$c$ condenser

cl contact line

cr critical

$d$ dry, disjoining

$e$ evaporator or evaporation

$f$ liquid film, film edge (contact line)

$f b$ feedback PHP section (vertical in Fig. 1)

$i$ bubble or plug identifier number

$K$ Kelvin

$l$ liquid

$m$ meniscus

nucl nucleation

$o$ outer tube wall

$p$ PHP spatial period or at constant pressure

$r$ reservoir, recoil (sec. 2.3.5)

$S$ Stokes

$s$ slip

sat saturation

$t$ total, tube

$V$ Voinov

$v$ vapor or at constant volume

$w$ internal tube wall

0 initial

\section{Introduction to the PHP predictive modeling}

One of the contemporary technological design challenges of many devices is a reduction of their mass. This is required 
either to reduce their energy consumption and $\mathrm{CO}_{2}$ emission or simply for convenience of their use. The designers thus have to replace metals by lighter synthetic materials, which, however, are poor heat conductors and thus require special thermal management solutions to evacuate the heat from the electronic components. On the other hand, because of device miniaturization, the conventional cooling technologies face challenges like the cooling heat fluxes of several hundreds of $\mathrm{W} / \mathrm{cm}^{2}$, long term reliability, and very low costs for consumer market products, among others. This necessitates more efficient novel cooling approaches. Special devices called heat pipes are used more and more widely to transfer the excessive heat to a colder environment.

A heat pipe is generally a sealed container tube filled with the working fluid. One side of this container (called evaporator) is brought in thermal contact with a hot point to be cooled. The other end (called condenser) is connected to a cold point where the heat can be dissipated. A portion between evaporator and condenser is called adiabatic section. The working fluid and the heat pipe regime are both chosen in such a way that the saturation temperature is in between the evaporator temperature $T_{e}$ and condenser temperature $T_{c}$. The fluid is thus vaporized in the evaporator. The generated vapor is transported by its expansion to the condenser section and condenses there. The heat is transferred due to absorption of the latent heat in the evaporator and its release in the condenser. Since the latent heat is generally large, the heat pipes are quite efficient. There are different kinds of heat pipes. They differ by their geometry and a mechanism of liquid transport from condenser to evaporator.

Pulsating heat pipe (PHP, called by some authors oscillating heat pipe), invented in the early 1990s [1] present a promising alternative for conventional heat pipes. PHP is a simple meandering capillary tube (Fig. 1). Because of capillarity, the vapor domains (that we call bubbles) are separated by the liquid plugs. When the temperature difference between evaporator and condenser exceeds a certain threshold, the bubbles and plugs begin to move spontaneously back and forth and penetrate both into the cooled portion of the tubes (condenser) and heated portion (evaporator). In addition to the latent heat transfer mechanism, the convective heat transfer occurs in PHP through the liquid plugs that accumulate the heat in the evaporator and release it in the condenser. This phenomenon is a reason of high efficiency of PHPs in comparison to other types of heat pipes. Another PHP advantage is a potentially higher dryout limit that is suggested by the difference of the CHF of the pool and convective boiling. Compared to other cooling solutions, PHPs are simple and thus more reliable and cheap. However their practical use is still hindered by the insufficient understanding of their functioning and the absence of reliable modeling approaches. For all the above reasons, PHP is an active field of research, with dozens of publications each year in leading scientific journals.

First of all, a few terms need to be defined because the same words are currently used in the literature with different meanings. By "turn" we mean a U-turn portion of the PHP channel. Number of PHP turns is the turn number on the evaporator side of the PHP meander, i.e. a half of the total number of turns. By "branch" we mean a tube section linking the centers of two neighboring turns. The total number of branches is twice the number of turns. For instance, the 5-turn PHP represented in Fig. 1 has 10 branches. The feedback section is a portion connecting the first and the last parallel PHP branches. It can be absent when PHP is folded in the 3rd dimension. By "evaporator (condenser) section" we mean a heated (cooled) portion of the PHP channel, respectively. In the conventional PHP topology (Fig. 1) there is a single evaporator and a single condenser section per branch; they can however be several per branch for the multi-source PHP. By "wall" we mean the internal channel surface.

There are three main PHP configurations. One distinguishes the closed-loop PHP shown in Fig. 1, which is recognized by many authors as the most efficient. For technological reasons, one sometimes uses the closed end PHP where the channel is closed at its both top and bottom right ends (in the orientation of Fig. 1), and there is no feedback section. Some research is done on the open end PHP where one or both ends are open into a reservoir that imposes the average pressure.

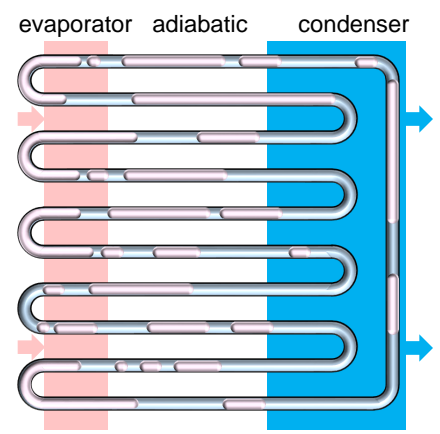

Figure 1: Schematic representation of the pulsating heat pipe (PHP) with the vapor bubbles and liquid plugs inside it. The heated (evaporator) sections of the tube are those inside the rose rectangle, while the cooled sections (condenser) are inside the blue rectangle. The tube sections in between are adiabatic.

One distinguishes two main PHP technologies: tubular and flat plate PHP. The tubular PHP is made of a capillary tube of circular cross-section. The channel of a flat plate PHP is usually engraved in a flat plate so the channel cross-section is often rectangular. As the two-phase flow in such channels is different from the flow in the cylindrical tubes, they should be modeled in a different way. Unfortunately, such a flow is not well studied. Unless mentioned otherwise, the discussion below applies to the cylindrical capillaries.

In the literature, one finds several attempts to apply the conventional approach of empirical correlations to evaluate the PHP performance, see e.g. [2-8]. Because of high number of physical parameters on which the PHP functioning depends (including the dimensionless parameters like liquid filling ratio, number of turns and inclination angle, which are especially difficult to implement in them) these approaches remain usually limited to the range of parameters for which they were developed. Because of the lack of PHP understanding, it is not even clear which dimensionless groups are the most important, and which groups can be omitted from the analysis. In some works, another empirical approach based on the training of the artificial neural networks (ANN) of different complexity is used [9-12]. 
In addition to the above mentioned shortcomings, a practical use of this method requires in addition the ANN model implementation.

In the past, the PHP functioning was reviewed by several authors [13-18] and in a book by Ma [19]. In this review we focus on the PHP hydrodynamic modeling that seems to be the only way to achieve a reliable PHP performance prediction for optimization of industrial prototypes. Only several 2D and 3D PHP models are available in the literature; they are reviewed in sec. 5 below. While they can give a valuable scientific information for the PHP understanding, they require so long computation time that they can hardly be considered as a design tool. For this reason the 1D modeling is the main focus of this review.

The author attempts to critically review here the existing PHP models and to show their relevance to the physical phenomena that occur inside the PHP channel. He hopes that such a physically based approach will contribute to the future development of the PHP technology.

\section{Physical phenomena and modeling of the PHP compo- nents}

The PHP functioning is intrinsically transient and even chaotic. For this reason it involves the physical phenomena which are either nonexistent or insignificant in conventional types of heat pipes, the functioning of which can be considered as stationary. To model the overall PHP functioning, one needs to start with understanding of these physical phenomena occurring inside the PHP channel in each of the PHP physical components: the vapor bubbles, the liquid plugs and the liquid films.

\subsection{Flow and heat exchange in the liquid domain}

To provide oscillations, the main flow regime that occurs inside the PHP corresponds to what is called slug-plug flow in the convective boiling. We use instead the bubble and plug terms used in the early PHP literature. In hydrodynamics (in particular, in microfluidics) such a flow is often referred to as the Taylor bubble flow, i.e. the flow of bubbles squeezed in a channel (Fig. 2a). Such a flow exists when the capillary forces are strong enough in comparison to the gravity that tends to create the stratified flow in the horizontal channel portions. This condition leads to the well-known limitation [13] on the internal channel diameter,

$$
d<d_{c r}=2 \sqrt{\frac{\sigma}{g\left(\rho_{l}-\rho_{v}\right)}} .
$$

This condition is necessary but however insufficient for the PHP oscillating functioning. The motion of the liquid plugs can be so fast that the inertial forces break their menisci eventually leading to the annular flow [20, 21], observed in the oneturn (more commonly called single-loop) PHP in the gravityassisted regime, which is in some respects similar to the thermosyphon operation.

There are many studies of constant velocity Taylor bubble flows, both theoretical, numerical, and experimental. These

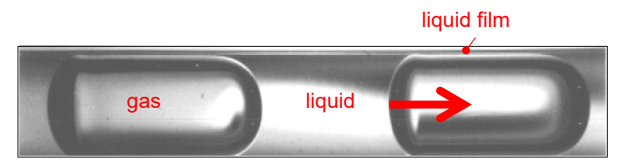

(a) Classical Taylor bubble flow (its direction is shown with an arrow).

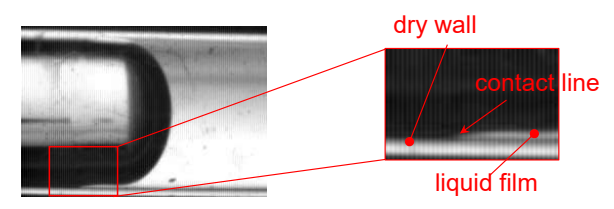

(b) Vapor bubble in the PHP evaporator [22].

Figure 2: Difference of conventional Taylor bubbles and bubbles in PHP.

studies are driven mainly by technological applications in microfluidics, chemical engineering, and thermal engineering, for the flow boiling heat exchangers. Starting from the articles by Bretherton [23], the main issues concern the pressure drop, heat exchange rate as a function of flow rate, void fraction, and the liquid film thickness. The latter issue will be addressed below, cf. sec. 2.3. When the film thickness is much smaller than the tube diameter, the flow inside the liquid plug is almost independent on the film presence or absence. Instead of addressing correlations for the pressure drop and heat exchange models that can be found in reviews (see e.g. [24-26]), we will discuss here the main physical features of the flow. This is necessary as the developed correlations may be invalid for the oscillating flow.

Consider first the pressure drop $\Delta p$ observed in the adiabatic constant velocity flow of a Taylor bubble. The liquid film around it is continuous. Since the pressure drop inside the vapor can be neglected, $\Delta p$ consists of two distinct components: that related to the menisci curvature and the viscous pressure drop in the liquid. The interfacial pressure jumps across the leading and trailing menisci of a bubble are proportional to their interfacial curvatures. If they were equal, this contribution would be zero. However, in reality the curvature of the leading meniscus is always larger than that of the trailing meniscus of a bubble (cf. Fig. 2a) so the meniscus contribution $\Delta p_{m}$ is always positive. The menisci curvature depends on the liquid film thickness deposited by the leading meniscus and "seen" by the trailing meniscus. Cherukumudi et al. [27] introduced a correction to the initial formula of Bretherton [23]. The pressure drop effect created because of the menisci curvature difference is

$$
\Delta p_{m}=14.56 C a^{2 / 3} \frac{\sigma}{d},
$$

where $C a=\mu V / \sigma$ is the meniscus capillary number. The expression (2) agrees with the numerical results [28].

\subsubsection{Pressure drop in the liquid plugs}

In spite of the smallness of the Reynolds number $R e=$ $V d / v$ for a capillary, the pressure drop in a liquid plug deviates from the Poiseuille expression because of the menisci influence. Consider the reference in which the mass center of liquid plug 
is immobile. The fluid is dragged by the moving wall. To provide the liquid mass conservation, the fluid velocity at the plug axis should be in the opposite direction, which means that rolls are created inside the plug (cf. [24-26] for an extended discussion). The flow change with respect to the Poiseuille flow is strong near the front and rear menisci but is small in the middle of the plug. Therefore, the overall drug is expected to contain a contribution from this effect independent of the liquid plug length $L_{l}$. According to the empirical correlation of Kreutzer et al. [28], the overall pressure drop in a cylindrical capillary contains the Hagen-Poiseuille term and a term independent of $L_{l}$ that accounts both for the modification of the plug flow near the menisci and for $\Delta p_{m}$ :

$$
\Delta p=\frac{16}{R e} \frac{\rho_{l} V^{2}}{r} L_{l}+5.44 R e^{1 / 3} C^{2 / 3} \frac{\sigma}{d} .
$$

The numerical coefficient and the $R e$ dependence provide the best fit both to the experimental data for air bubbles in several liquids and to numerical data.

In the remaining part of this section, we discuss some aspects of the single phase flow in a tube without the influence of the menisci (i.e. only the first term in Eq. 3). First we address the impact of oscillations. They impose a relevant length scale, which is the Stokes length $\delta_{S}=\sqrt{2 v / \omega}$. One argues in terms of two dimensionless numbers, either the kinetic Reynolds number $\operatorname{Re}_{\omega}=d^{2} \omega / v$ or the Womersley number $W_{o}=r \sqrt{\omega / v}$. The transition number $\beta=2 A \sqrt{\omega / v}$ is used to define the transition from laminar to turbulent flow that occurs when $\beta$ attains a value close to 700 [29]. The PHP flow is usually below this limit, hence one can consider only the laminar case. During os-

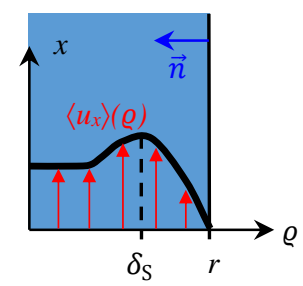

Figure 3: Sketch of the laminar oscillating flow pattern. The radial profile of the averaged in time root mean square axial fluid velocity is shown.

cillations, a boundary layer of the thickness $\delta_{S}$ develops if it is small with respect to the tube radius (i.e. for $W o \ll 1$ which corresponds to a small frequency) and the bulk fluid oscillates as a whole [30], with $\pi / 2$ phase shift with respect to the pressure. Maxima of velocity $u_{x}$ occur at a distance $\sim \delta_{S}$ from the wall; this phenomenon is referred to as the Richardson annular effect.

One is interested in the force $F=S \Delta p$ exercised by the fluid on the walls. $F$ can be obtained from the solution of the Navier-Stokes equations with the imposed oscillating pressure $[18,30]$ as the tangential component of shear stress integrated over the wall area. Because of the phase shift mentioned above, the problem can be solved with the Fourier transform so $\tilde{F}$ (the
Fourier transform of $F$ ) is complex. For a cylindrical capillary,

$$
\begin{aligned}
\tilde{F} & =-\left.\pi d \mu L_{l} \frac{\partial \tilde{u}_{x}}{\partial \varrho}\right|_{\varrho=r}=2 \pi \mu L_{l} \tilde{V} B(W o), \\
B(W o) & =W o \sqrt{i}\left(\frac{I_{0}(W o \sqrt{i})}{I_{1}(W o \sqrt{i})}-\frac{2}{W o \sqrt{i}}\right)^{-1} \\
& =4+\frac{i W o^{2}}{6}+O\left(W o^{4}\right),
\end{aligned}
$$

where $u_{x}$ is the axial fluid velocity and the approximation for small Wo is given in Eq. (5). By performing the inverse Fourier transform, one gets the corresponding two terms

$$
F=8 \pi \mu L_{l} V+\frac{1}{3} m_{l} \dot{V},
$$

where $m_{l}=L_{l} S \rho_{l}$ is the plug mass. The first term is the HagenPoiseuille expression, and the second presents the first-order oscillation correction. Its particularity is that it is independent of viscosity and corresponds to the "added mass" of $m_{l} / 3$. In other words, at small frequencies, one can consider that the entire liquid plug oscillates as a solid mass, by $1 / 3$ larger than its actual mass.

One can include now the impact of turbulence at large $R e$. By returning back to the constant velocity flow, one can use for this the well-known Blasius correlation

$$
\begin{aligned}
& F=C \pi r \rho_{l} V^{2} L_{l}, \\
& C= \begin{cases}16 / \operatorname{Re}, & 0<\operatorname{Re}<1180 \\
0.079 \operatorname{Re}^{-0.25}, & \operatorname{Re} \geq 1180\end{cases}
\end{aligned}
$$

used in many PHP works following that of Shafii et al. [31]. In the first line of this equation one recognizes the Hagen-Poiseuille expression.

The reduced 1D equation of motion of a liquid plug (which is in fact the Navier-Stokes equation integrated over the channel cross-section) is [32]

$$
\frac{\mathrm{d}}{\mathrm{d} t}\left(m_{l} V\right)=\left(p-p_{\text {next }}\right) S-F-F_{\text {turn }}+G
$$

where the 1.h.s. is simply the plug momentum derivative, $G$, the gravity force. The pressures $p$ and $p_{\text {next }}$ correspond to the ends of the plug ("next", in the direction of the $x$ axis). The menisci-related second term of Eq. (3) is sometimes included into $F[33]$.

The pressure loss in the PHP tube turns may be important, so a supplementary force $F_{\text {turn }}$ comes from a curved tube. Again, we discuss here the well-studied case of constant $V$. The $F_{\text {turn }}$ term was first introduced into the PHP modeling by Mameli et al. [34] using the 3-K Darby method [35] for its calculation. We recommend a different approach since only a part of liquid plug of the length $L_{l, t u r n} \leq L_{l}$ may be present in the tube bend. The turn angle $\varphi=L_{l, \text { turn }} / r_{\text {turn }}$ of the plug (where the turn radius $r_{\text {turn }}$ is measured along the tube axis line) may vary and be different from the discrete values $90^{\circ}$ or $180^{\circ}$ considered by Darby. For this reason, the following approach seems to be 
more appropriate for the PHP case. Idelchik [36] defines the additional force of the inertial origin as

$$
F_{\text {turn }}=0.105 S \rho_{l} V^{2} A_{\text {turn }}(\varphi) \sqrt{d / r_{\text {turn }}}
$$

where the $A_{\text {turn }}(\varphi)$ function is nonlinear

$$
A_{\text {turn }}(\varphi)= \begin{cases}1, & \varphi=\pi / 2 \\ 0.9 \sin \varphi, & \varphi<\frac{7}{18} \pi \\ 0.7\left(1+\frac{\varphi}{\pi}\right), & \varphi>\frac{10}{18} \pi\end{cases}
$$

Such an approach is used in CASCO (sec. 4.2).

\subsubsection{Non stationary and non-adiabatic Taylor bubble flow}

There are several aspects of this problem. First, one notes the dependence of the Nusselt number on the plug length $L_{l}$ for the unidirectional laminar single-phase flow, in analogy with the problem of entry of a fluid into an isothermal tube of a different temperature solved by Graetz. The Nusselt number in this case depends on the Prandtl number $\operatorname{Pr}=v / D_{l}$ and on the dimensionless quantity $L^{*}=L_{l} /(d \operatorname{Pr} R e)$. For $\operatorname{Pr} \gg 1$ (which is an acceptable assumption e.g. for water or ethanol) the Hausen approximation can be used

$$
N u_{l}=3.66+\frac{0.075 / L^{*}}{1+0.05 L^{*-2 / 3}} .
$$

The second aspect of this problem concerns the heat exchange in oscillating single phase flow, where oscillations also leads to the $N u_{l}$ increase. It has been actively studied since a work of Kurzweg and de Zhao [37] who developed an analytical approximate theory (focused however on the axial, not radial, heat exchange). We note the more recent works [38-43]. Most of these studies were reviewed recently by Ma [19]. In particular, it has been shown that the Nusselt number varies around the constant heat flux laminar flow value $N u_{l}=4.36$, but the variation is small, so this value appears to be a good approximation. This data has also been confirmed experimentally in a square channel by Mehta and Khandekar [44]. Some modeling approaches use however more elaborate correlations [45] accounting for the turbulent flow [46].

The heat exchange at the Taylor bubble flow conditions is of interest for several fields like chemical processing, flow boiling and microfluidics. After a review by Talimi et al. [25], one can mention the experimental and numerical studies [47-51] for the unidirectional plug flows and the oscillatory flow studies $[44,52]$. The wall heat exchange can be seen as a sum of contributions from the liquid plug as discussed next, the liquid film (cf. sec. 2.3) and the vapor (cf. sec. 2.2.1).

\subsubsection{Heat transfer modeling in PHP}

Within the Lagrangian formulation, in the reference of the moving plug $x^{\prime}$, the heat exchange in the liquid is usually assumed to obey the $1 \mathrm{D}$ thermal diffusion equation

$$
\frac{\partial T_{l}}{\partial t}=D_{l} \frac{\partial^{2} T_{l}}{\partial x^{\prime 2}}+\frac{\wp U_{l}}{\rho_{l} c_{l} S}\left[T_{w}\left(x^{\prime}\right)-T_{l}\left(x^{\prime}\right)\right],
$$

where $\wp$ is a channel perimeter $(\wp=\pi d$ for a cylindrical tube), and the heat exchange coefficient $U_{l}=N u_{l} \lambda_{l} / d$ is given with $N u_{l}$ discussed above. A delicate part is the numerical projection of $T_{w}(x)$ defined in the reference of the PHP channel to the moving plug reference $x^{\prime}$.

One mentions that the heat advection inside the plug is neglected in Eq. (11). This is justified by the fact that, in general, the channel is thin, so the radial heat exchange (last term) is much stronger than the axial advection.

The saturation temperature at the liquid meniscus

$$
T_{l}\left(x^{\prime}=0, L_{l}\right)=T_{\text {sat }}(p) .
$$

provides both boundary conditions for the plugs of a multibranch PHP.

For a PHP open to a reservoir (cf. Fig. 20 below), one needs another boundary condition from the reservoir side of the plug. The thickness of thermal boundary layer formed near the liquid meniscus is of the order $\sqrt{D_{l} / \omega_{0}}$, where $\omega_{0}$ is the frequency of oscillations. This thickness is assumed to be much smaller than the liquid penetration length into the adiabatic section. Such an assumption is often justified for the single-branch PHP (cf. sec. 3 below). This means that in the case of imposed wall temperature $T_{c}$ in the condenser, the liquid temperature inside it is also equal to $T_{c}$ and

$$
T_{l}(x \in \text { condenser })=T_{c} .
$$

With these assumptions, the integration interval may be reduced to the adiabatic and evaporator sections.

One notes that in the case of imposed temperatures both in the condenser and evaporator sections, the liquid temperature impacts neither the vapor bubble state nor the overall PHP dynamics. To determine the global PHP heat transfer, Eq. (11) is solved a posteriori, after the plug dynamics calculations [31].

When the thermal fluid-solid coupling is introduced, the fluid dynamics should be solved together with the liquid and solid heat exchange at each time step. Evidently, this makes the problem more complicated. The temperature distribution along the internal tube wall is described by the equation [46]

$$
\frac{\partial T_{w}}{\partial t}=D_{w} \frac{\partial^{2} T_{w}}{\partial x^{2}}+\frac{j_{w}}{\rho_{w} c_{w}},
$$

where $x$ is a coordinate that varies along the PHP channel. Since the temperature of the condenser sections is often imposed, Eq. (14) is solved within the evaporator and adiabatic sections. The temperature is assumed to be constant $T_{w}(x)=T_{c}$ within the condenser. The effective volume heat source is

$$
j_{w}=\frac{1}{S_{w}} \begin{cases}q_{e} \wp_{o}-q_{\text {fluid }} \wp & \text { if } x \in \text { evaporator, } \\ -q_{\text {fluid }} \wp & \text { if } x \in \text { adiabatic section, }\end{cases}
$$

where $S_{w}=\pi\left(d_{o}^{2}-d^{2}\right) / 4$ is the tube material cross-section area and $\wp_{o}=\pi d_{o}$ is the outer tube perimeter for the cylindrical capillary case. In the problems with smooth temperature distribution along the channel, one usually assumes that the heat flux $q_{e}$ applied to the evaporator is distributed uniformly,

$$
q_{e}=\frac{P_{e}}{\wp_{o} L_{e}} .
$$


The heat flux $q_{\text {fluid }}=U_{\text {fluid }}(x)\left[T_{w}(x)-T_{\text {fluid }}(x)\right]$ is transferred from the internal tube wall to the fluid. It varies both in time and space. The definitions of $T_{\text {fluid }}$ and $U_{\text {fluid }}$ are

$$
\begin{gathered}
T_{\text {fluid }}(x)= \begin{cases}T & \text { if } x \in \text { dry wall, } \\
T_{\text {sat }} & \text { if } x \in \text { liquid film, } \\
T_{l}(x) & \text { if } x \in \text { liquid plug, }\end{cases} \\
U_{\text {fluid }}(x)= \begin{cases}U_{v} & \text { if } x \in \text { dry wall, } \\
U_{f} & \text { if } x \in \text { liquid film, } \\
U_{l} & \text { if } x \in \text { liquid plug. }\end{cases}
\end{gathered}
$$

One can see that, unlike the imposed wall temperature case, the liquid temperature influences the meniscus dynamics through the wall temperature variation during oscillations.

The boundary conditions for the wall thermal problem need now be defined. One often assumes that the condenser is efficient enough to impose a constant temperature $T_{c}$ at its boundary,

$$
T_{w}(x \in \text { condenser })=T_{c} .
$$

Sometimes the cooling efficiency cannot be assumed infinite so the temperature of condenser sections varies. One introduces in this case a (high) heat transfer coefficient $U_{\infty}$ toward the environment (e.g. via the external air cooler) of a temperature $T_{\infty}$. In this case Eq. (14) applies in the condenser too. One defines

$$
j_{w}=\frac{1}{S_{w}}\left\{\wp_{o} U_{\infty}\left[T_{\infty}-T_{w}(x)\right]-q_{\text {fluid }} \wp\right\}
$$

in the condenser section.

\subsection{Physics and modeling of vapor phase}

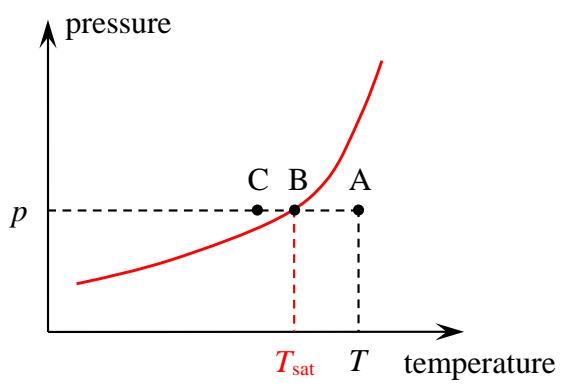

Figure 4: The sketched saturation curve and vapor thermodynamic states. The saturated vapor with the temperature $T_{\text {sat }}$ corresponds to the point $\mathrm{B}$. The superheated vapor (or gas) corresponds to the point $\mathrm{A}$. The vapor in the state of supersaturation is shown by the point $\mathrm{C}$.

The vapor thermodynamics is a key issue for the PHP theoretical description because the vapor plays the role of a spring necessary for oscillations. A naïve idea on the vapor state comes from the analogy with the conventional heat pipes where the vapor is at saturation temperature $T_{s a t}(p)$, corresponding to its pressure $p$ (point B in Fig. 4). Alternatively, the vapor state can be in the gas region of the phase diagram, which means that it is superheated with respect to $T_{s a t}$, i.e. its temperature $T>T_{\text {sat }}(p)$. The metastable (supersaturation) vapor state (point
C in Fig. 4) is not likely to be achieved in PHP where the presence of liquid menisci should cause immediate condensation on them even at a tiny supersaturation. A caused by such supersaturation homogeneous fog-like nucleation of liquid droplets in the vapor is not likely to appear either because it requires overcoming an additional energy threshold [53].

For the PHP modeling, two aspects should be considered: static and dynamic. First, one needs to understand, which vapor state (saturated or superheated) occurs for each particular set of thermodynamic variables ( $T$ and $p$ ), i.e. the equation of state (EOS). Second, one needs to define a law for their variation.

\subsubsection{Vapor thermodynamic state}

To distinguish between the saturated and superheated vapor states, simultaneous measurements of the vapor temperature and pressure in the functioning single branch PHP have been carried out by Gully et al. [54]. The transient vapor temperature measurement is not an easy task because of the weak heat exchange between a temperature sensor and the vapor. It is important to install a microscopic thermocouple $(\mu \mathrm{TC}, \varnothing 30 \mu \mathrm{m}$ in this work) inside the vapor phase in such a way that it is never wetted: a covered by a thin liquid film $\mu \mathrm{TC}$ should measure $T_{\text {sat }}$ whatever is the vapor temperature. To reduce the parasite heat exchange (in particular, the radiative exchange with the internal tube walls), the working fluid was oxygen at about $70 \mathrm{~K}$. The $\mu \mathrm{TC}$ radiative heat exchange was carefully evaluated. It was found to be much smaller than the convective heat exchange with the vapor.

The results are presented in Fig. 5. The temperature $T_{\mu \mathrm{TC}}$ measured by the micro-thermocouple is compared to $T_{s a t}(p)$ calculated from the measured evolution $p(t)$ of the pressure in the vapor bubble. Since the pressure oscillates around $p_{r}^{H}\left(=p_{r}\right.$ plus the hydrostatic pressure caused by the liquid column), the saturation temperature oscillates around $T_{\text {sat }}\left(p_{r}^{H}\right)$. The $\mu \mathrm{TC}$ measurements (Fig. 5a) reveal several important features. First, $T_{\mu \mathrm{TC}}$ oscillates around the evaporator temperature $T_{e}$ which shows the importance of heat exchange between the vapor and dry channel wall in the evaporator. Second, $T_{\mu \mathrm{TC}}$ remains always larger or equal to $T_{\text {sat }}(p)$ which means that the vapor can always be considered as superheated (cf. Fig. 4). The most striking feature is that the vapor sometimes becomes hotter than the wall that heats it, which is caused by the vapor compression. A comparison with the theoretical modeling (Fig. 5b) suggests that the $\mu \mathrm{TC}$ does not resolve rapid temperature variations, in spite of substantial efforts to reduce its thermal inertia (by reducing its size and performing experiments at cryogenic temperatures where the heat capacity is small).

The vapor temperature was analyzed by two more groups, in both cases above the room temperature. Rao et al. [55] measured the vapor temperature $T$ in the single branch PHP. $T$ was almost constant (probably because of the strong radiative heat exchange) and close to $T_{e}$, which suggests the superheated state in the agreement with the Gully et al. results.

Noh and Kim [56] have measured simultaneously the vapor temperature and pressure in the two-branch tubular transparent PHP in the vertical orientation operating in the circulation 


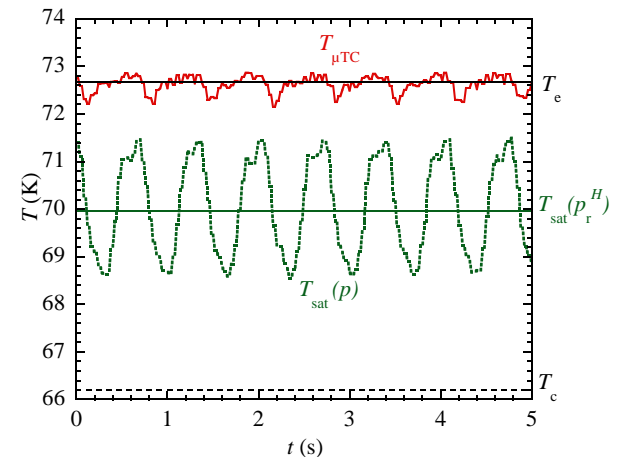

(a) Experimental data.

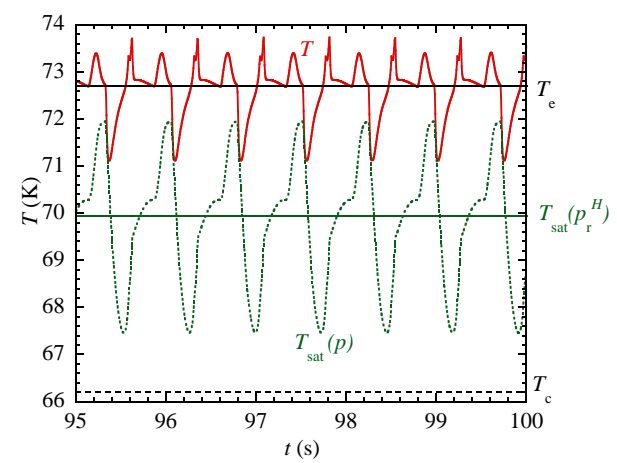

(b) Corresponding numerical simulation performed with the film evaporation-condensation model (sec. 3.2).

Figure 5: Results of the cryogenic single branch PHP experiment and corresponding modeling [54].

regime. They concluded that the vapor bubbles were all at saturation and the pressure of all the bubbles was almost identical. It was not however clear how it was possible under the continuous motion conditions (what was the moving force) accounting for the fact that some of the bubbles situated in the condenser, and some in the evaporator. Under such a regime, a continuous rewetting of the thermocouples should have occurred, which was likely to cause such a result.

In the subsequent work of the same group, the vapor temperature measurement in the transparent multi-branch PHP was undertaken [57] under the regime of small amplitude oscillations. They could know the meniscus position and the film edge position with respect to two $\mu \mathrm{TCs} \varnothing 50 \mu \mathrm{m}$ situating in the adiabatic and in the evaporator sections. They have recorded the $\mu \mathrm{TC}$ temperature during both vertical and horizontal PHP operation. The results in vertical orientation are especially interesting because the evaporator $\mu \mathrm{TC}$ is rarely wetted. They have concluded that when the $\mu \mathrm{TC}$ faced the dry wall, its temperature was above $T_{\text {sat }}$ so the vapor was superheated. However, when $\mu \mathrm{TC}$ faced the liquid film, its temperature dropped to $T_{\text {sat }}$ thus suggesting a strong temperature heterogeneity inside the vapor. Unfortunately, the thermal analysis of $\mu \mathrm{TC}$ was not reported, so an impact of strong radiative heat exchange between the $\mu \mathrm{TC}$ and the wall could not be ruled out. The history of the events is not sufficiently described either, so one cannot judge if the equality $T_{\mu \mathrm{TC}}=T_{\text {sat }}$ appears because of the wetting film covers $\mu \mathrm{TC}$ or not.

Most simulation approaches discussed below describe the vapor by a unique temperature. It is however evident that the vapor temperature is not entirely homogeneous during its compression or expansion. Thermal boundary layers are developed near the dry walls at temperature $T_{w}$ and the liquid films at temperature $T_{\text {sat }}$ covering the walls. The thickness of these boundary layers is a complex yet unsolved issue. Direct numerical simulations [54] of the gas flow during oscillatory compression performed with ANSYS CFX software for oxygen at about $70 \mathrm{~K}$ showed a thickness of $\sim 0.25 \mathrm{~mm}$ in the tube of $d=2 \mathrm{~mm}$ diameter at the frequency of $1.5 \mathrm{~Hz}$. The vapor flow remained mostly laminar in the thermal boundary layers; turbulent mixing was absent near the wall. For this reason, in one-dimensional models one can reasonably assume that the vapor bulk can be described by a unique temperature $T$. The heat exchange coefficient is $U_{v}=N u_{v} \lambda_{v} / d$. The heat exchange rate with the dry wall area of the length $L_{v}$ reads

$$
\dot{Q}_{v}=U_{v} \wp L_{v}\left(T_{w}-T\right) .
$$

The above numerical calculation have resulted in the average value $N u_{V} \simeq 6$.

In their pioneering article on the PHP modeling, Shafii et al. [31] assumed that the vapor was superheated and thus behaved like the ideal gas with the EOS

$$
p=m R_{v} T / \Omega
$$

It is well known to provide an elastic response (pressure rises at compression or reduces at dilatation) needed for oscillations. This equation is quite precise for the real fluids, even in a close vicinity of saturation state [58] so there is no need to use more complex EOS. At the same time, one needs to introduce the phase change. In their model, Shafii et al. [31] assumed that the phase change rate $\dot{m}$ is proportional to the difference $T_{w}-T$ of the wall and vapor temperatures. The same assumption has been adopted later by many other teams (cf. sec. 2.3.6). This assumption has been justified by the fact that the evaporation flux is provided by the film evaporation/condensation which is well known to be proportional to the $T_{w}-T_{\text {sat }}$ (cf. Eq. (34) and the associated discussion). Shafii et al. have postulated that the vapor was at saturation so, according to them, $T=T_{\text {sat }}$, which justified the phase change rate proportional to $T_{w}-T$. The assumption $T=T_{\text {sat }}$ however contradicts Eq. (21) and thus should be abandoned. Indeed, the pressure of saturated vapor is entirely defined by its temperature (Fig. 4) rather than by Eq. (21). Because of this difference, the vapor cannot be simultaneously and continuously both at saturation and superheated states, so the assumption $\dot{m} \sim\left(T_{w}-T\right)$ is hardly valid.

In an alternative approach, d'Entremont and Thome [59, 60] assume the vapor to always remain at saturation. During the volume variation at oscillations, vapor density $\rho_{s a t}$ changes and they find a new vapor temperature by inverting the $\rho_{\text {sat }}(T)$ curve. We note that the saturated vapor model also creates a kind of elastic response: when the vapor volume reduces at constant mass, $\rho_{\text {sat }}$ increases (i.e. $\rho_{\text {sat }}^{-1}$ decreases) and the pressure 
rises together with the vapor temperature by following the saturation curve. Note that the such an "automatic" temperature change at compression/dilatation is hardly justified because the associated vapor energy change is not balanced. In a more realistic model, the vapor temperature change should be defined from the vapor energy balance that includes the heat exchange with the environment, see sec. 2.2.2.

According to the above experimental results, the vapor is mainly superheated. However it can certainly fall into saturation state in some specific situations, e.g. during compression preceding the bubble disappearance, so both states should be modeled in a PHP simulation. Such a model have been suggested by Nikolayev and Nekrashevych [58].

\subsubsection{Vapor energy equation}

The vapor dynamics is described by its energy balance that should be written starting from the first law of thermodynamics. When the vapor is superheated and obeys the ideal gas law (21), Shafii et al. [31] showed that it can be described by the equation (see [61] for its detailed derivation)

$$
m c_{v v} \dot{T}=R_{v} T \dot{m}-p \dot{\Omega}+\dot{Q}_{v}
$$

where $c_{v v}$ is the vapor specific heat at constant volume and dot means the time derivative. If one neglects $\dot{Q}_{v}$, this equation can be reduced by using both EOS (21) and Mayer's relation $c_{p v}=c_{v v}+R_{v}$ valid in the ideal gas approximation:

$$
\frac{\dot{T}}{T}=\frac{\gamma-1}{\gamma} \frac{\dot{p}}{p}
$$

The advantage of this representation is that it can be integrated directly [62],

$$
p \sim T^{\frac{\gamma}{\gamma-1}} .
$$

This approximation is convenient because reduces by one the number of differential equations per bubble that need to be solved numerically. It was used widely in the PHP modeling as it can be justified: the contribution of $\dot{Q}_{v}$ into the overall heat transfer is quite small in the stable oscillation regime. In some regimes however $\dot{Q}_{v}$ is important. In particular, it will be shown in sec. 3.2.1 below that it is essential for the startup threshold determination. $\dot{Q}_{v}$ is thus important for the modeling of intermittent oscillation regime where PHP stops and restarts again.

There was a lot of controversy concerning the vapor energy equation starting with Dobson [63] who replaced $R_{v} T$ in Eq. (22) by the vapor enthalpy $h_{v}$. This is obviously incorrect because $h_{v}$ is defined within an arbitrary constant, see [61]. In a work [64], the vapor is treated as if it was solid $\left(m c_{p v} \dot{T}=\dot{Q}_{v}\right)$, with nor pressure work neither phase change account. Another incorrect (because containing $h_{v}$ defined within a constant) vapor energy equation

$$
\frac{\mathrm{d}}{\mathrm{d} t}\left(h_{v} m\right)=\dot{Q}_{v}
$$

has been used in the simulation work of Holley and Faghri [46] and later, in the simulations of Mameli et al. [34] based on a similar approach. More recently, Manzoni et al. [65, 66] used a slightly different equation (Eq. (27) of [65]) that can be rewritten as

$$
m \dot{h}_{v}=\dot{m} \mathcal{L} .
$$

Such an equation means a huge energy (i.e. latent heat) pumping into the vapor and thus leads to its strong mechanical response. This is obviously incorrect because, instead of increasing the vapor enthalpy, the latent heat released in condensation should be absorbed by the cooler.

In all of the above cited works, each vapor bubble was described with a unique pressure and temperature value (which is a OD approach). Senjaya and Inoue [67] solve the 1D (axial) inviscid compressible flow within each vapor bubble with the space dependent variables ( $p, T$, velocity, etc.). The ideal gas EOS (21) is used. The radial distribution of the vapor velocity is not calculated but accounted for via a friction force between the liquid film and the vapor determined with the help of the Wallis correlation [53]. The mass and energy continuity equations account for variation of the vapor mass flux $J$ along the liquid film that is assumed continuous within the bubble. Such an approach is probably more accurate but requires much more computer resources. Some multidimensional approaches (sec. 5) solve for the $2 \mathrm{D}$ or $2 \mathrm{D}$ axisymmetric vapor flows which are even more computationally expensive than the above $1 \mathrm{D}$ formulation.

\subsection{Liquid film dynamics in PHP}

The adequate modeling of liquid films is extremely important. As in other kinds of heat pipes, an important part of the heat and mass transfer transits through the liquid films. This issue becomes of prime importance in PHP because the mass transfer controls the oscillation dynamics.

In the early period of PHP studies, many researches considered that the film heat exchange is not a major contributor to the overall exchange. For this reason, early simulation approaches [19, 34, 46, 62, 68-70] based on [31] have not described the films at all. It was considered that the heat convection via the liquid plug motion is much stronger than the latent heat exchange. First film-based simulations [32] have predicted however that it is not so and the latent heat exchange via the films is actually stronger. These findings where confirmed by more recent in-situ experimental measurements [71, 72] and simulations [73].

The liquid films in PHP are entirely different from their counterparts in the capillary heat pipe. In the latter, the films are wetting films that appear because of the intermolecular (van der Waals) attraction forces often described in terms of the disjoining pressure. While the typical thickness of wetting films is $10-100 \mathrm{~nm}$, the thickness of PHP films is much larger, 10$100 \mu \mathrm{m}$ [74-76]. For this reason, early modeling of PHP films $[77,78]$ as wetting films is not adequate. The liquid films in PHP (cf. Fig. 6) are of purely hydrodynamic origin and appear as a result of deposition by the receding liquid menisci.

The hydrodynamic theory of thin liquid films is well established. Because of their small thickness, the Reynolds number is low, and the film deposition can be described in lubrication 


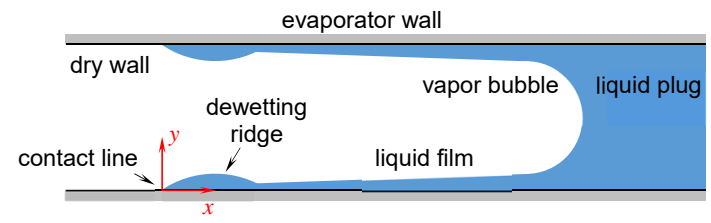

Figure 6: Sketch of the experimentally discovered liquid film shape in the PHP evaporator [75].

approximation of the hydrodynamics equations $[79,80]$ that assumes a small interface slope. Such an approach results in a nonlinear equation

$$
\frac{\partial \delta}{\partial t}-\frac{\partial}{\partial x}\left(\frac{\delta^{3}}{3 \mu} \frac{\partial p_{l}}{\partial x}\right)=-\frac{J}{\rho_{l}}
$$

Its strong advantage over the conventional hydrodynamics equations is evident: it includes the film thickness as an explicit variable. The liquid pressure $p_{l}$ at the liquid-vapor interface is linked to its local curvature $K$ in the axial plane with the Laplace equation

$$
\Delta p^{i} \equiv p-p_{l}=\sigma\left(K+\frac{1}{r-\delta}\right) \simeq \sigma\left(K+\frac{1}{r}\right) .
$$

Here, $K \simeq \partial^{2} \delta / \partial x^{2}$; the vapor pressure $p$ equilibrates very quickly, so its contribution to Eq. (25) is negligible (just like that of $r^{-1}$ term omitted hereafter).

A general feature of the thin film flows is their high hydraulic resistance (responsible, in particular, for the lubrication phenomena). For this reason, one can consider that there is no axial flow in the film, except in the regions where the curvature $K$ varies strongly, i.e. near the CL and the meniscus.

\subsubsection{Deposition of liquid films}

Before discussing the effect of mass exchange and oscillations, the film deposition under adiabatic conditions needs to be understood. Therefore we consider $J=0$ in this section. The film deposition was first described by Landau and Levich [81] and rederived later by Bretherton [23] for the case of Taylor bubbles (Fig. 2a).

The liquid film appears as a result of competition between the viscous and capillary forces in a transition region adjacent to the liquid meniscus where a curvature gradient occurs (Fig. 7a). The viscous friction tends to immobilize the fluid near the tube wall and thus to increase the film thickness. The surface tension tends to decrease the meniscus area thus making its radius of curvature as large as possible, which causes reduction of the film thickness. Such a competition is characterized by the meniscus capillary number $\mathrm{Ca}$. The deposited film thickness is controlled by the meniscus curvature and can be evaluated as

$$
\delta_{0}=\frac{0.67 d C a^{2 / 3}}{1+3.13 C a^{2 / 3}+0.504 C a^{0.672} R e^{0.589}-0.352 W e^{0.629}} .
$$

according to the correlation of Han and Shikazono [83]. The denominator can be seen as a correction appearing because of the meniscus curvature difference from $2 / r$ [84]. The presence

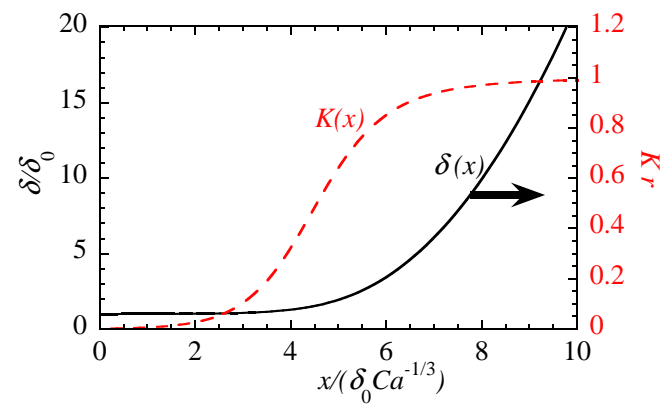

(a) Shape at liquid receding and corresponding variation of interfacial curvature $K$ (dashed line). The curvature decreases monotonously from the meniscus curvature to zero towards the flat film.

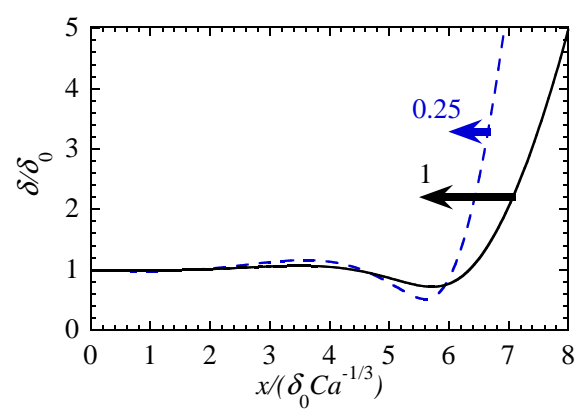

(b) Shape at liquid advancing over the preexisting liquid film of thickness $\delta_{0}$ for two different advancing velocities. The curve parameter is the ratio of the advancing velocity and the receding velocity having used to deposit the film. $\mathrm{Ca}$ in the abscissa scaling corresponds to the receding velocity.

Figure 7: The liquid-vapor interface shape in the transition region between the liquid film and the spherical meniscus [82]. The reference system is shown in Fig. 6; zero of $x$ axis is chosen arbitrarily. Arrows indicate the direction of plug motion.

of the Reynolds number and Weber number $W e=d \rho_{l} V^{2} / \sigma$ accounts for the inertial effects.

It is important to mention that the film deposition depends on the wetting properties. Snoeijer et al. [85] show that the film is deposited beginning from a threshold $C a$ value $C a_{c r}$, which thus defines the dynamic wetting transition. For complete wetting conditions, $C a_{c r}=0$ and increases with the contact angle [86]. Fig. 8 shows that the CL velocity is equal to the meniscus velocity (i.e. $C a_{c l}=C a$ ) when $C a<C a_{c r}$, which means the absence of film deposition. The meniscus is basically spherical, and shows an apparent contact angle $\theta_{\text {app }}$ defined by the microscopic effects acting in the CL vicinity (see sec. 2.3.3 for more detailed discussion). Note that $\theta_{a p p}$ depends on the contact line capillary number $C a_{c l}=\mu V_{f} / \sigma$, where $V_{f}$ is the CL velocity defined to be positive at receding. $C a_{c r}$ corresponds to the value of $C a_{c l}$ where $\theta_{a p p}$ becomes zero [87]. For $C a \geq C a_{c r}, C a_{c l}$ becomes smaller than $C a$, which indicates the film deposition. Note a jump of $C a_{c l}$ right after the deposition threshold that appears because, at film deposition, a dewetting ridge (Fig. 6) is formed near the contact line and $\theta_{a p p}$ becomes to be defined by its hydrodynamics rather than by that of the meniscus [88].

Receding of meniscus with no film [14] is rarely encoun- 


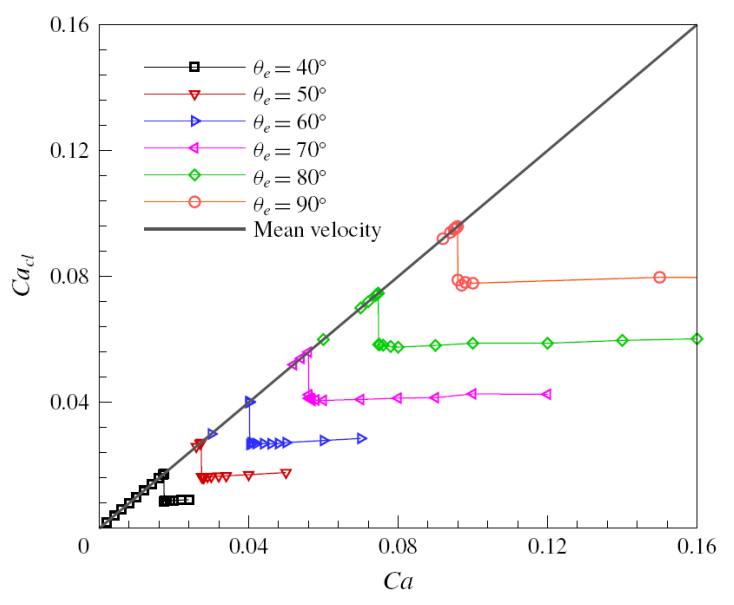

Figure 8: Variation of $C a_{c l}$ as a function of the meniscus receding capillary number $\mathrm{Ca}$ according to the numerical simulation by the diffuse interface method performed for the cylindrical capillary (axisymmetric) for different values of static contact angle. (Reprinted from Ref. [86] with permission from Cambridge University Press)

tered in PHP because $\mathrm{Ca}$ is usually large enough and the tube is wettable.

One needs to point out the effect of wall defects that can be caused either by its roughness or chemical heterogeneity. The heterogeneity leads to the wetting hysteresis. It is a range of contact angles, for which the contact line remains immobile [89]. To provide the liquid receding, the contact angle should be smaller than a value called the receding contact angle; for CL advancing, the contact angle should be larger than a value called the advancing contact angle. In between, the CL is pinned on the defect borders and the contact angle is defined by the liquid interface shape [90]. Similarly to the statics, the CL dynamics is also impacted by defects. Instead of being smooth, the stickslip motion occurs microscopically [91]: some parts of CL are intermittently pinned (stick to the defects) and then rapidly displace (slip) to a new pinning position. However, if the wall quality is good enough, such a motion can still be considered as macroscopically smooth.

The case of the rectangular capillaries is extremely important for the flat plate PHP. It is generally believed in PHP community that like in the static film drying, the rectangular geometry favors the film formation in the corners by capillarity so the film thickness has always a minimum in the middle of the side of the rectangle so the drying should occur there. Detailed experimental observations in the transparent PHP evaporator of rectangular cross-section with a high aspect ratio $(2 \times 22 \mathrm{~mm})$ show however an opposite tendency.

In Fig. 9(a-e), one can see the meniscus receding (a thick curve) and the dynamics of CLs on both front and rear portholes of the evaporator. Later on (Fig. $9 \mathrm{f}-\mathrm{j}$ ), the meniscus disappears from the view field. The portholes are heated with a transparent resistive (ITO) film. The grid of parallel wires that situates behind the evaporator serves for the optical film profile measurement. The rear porthole (blue CL contours) is heated stronger and the film on it disappears quicker. Let us consider its dynamics. Initially the CL is of U-shape as expected (Fig.
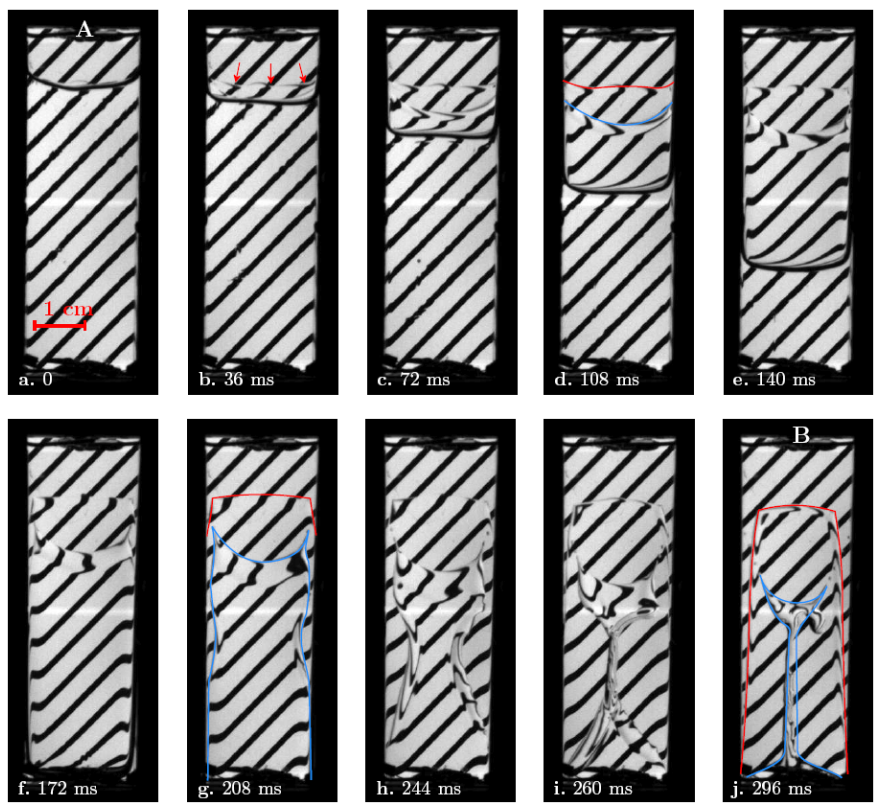

Figure 9: Film dynamics after the film deposition by the receding meniscus on two sides of a rectangular transparent PHP evaporator [92]. Blue contours: CL on the hotter rear porthole; red contours: CL on the front porthole.

9b-d). Then the film ruptures near the corners (Fig. 9e) and the CL moves from the corners to the center (Fig. 9j). Same thing (but later, cf. Fig. 9g) happens to the front film (red CL contours). The film takes a shape of a tongue and there is no more film in the corners. This feature can be explained by the theory of film deposition dynamics in the square capillary. In dynamics, the film thickness is actually smaller not in the center but near the corners [93]. This is where the evaporation is the strongest, so the rupture should indeed occur there.

Such tongue-shaped films lead to the reduction of evaporation area. It is probably one of the reasons of a poorer performance of the flat plate PHP in comparison with its tubular counterpart [94]. The flat plate PHP is however more convenient from the technological viewpoint (fabrication and integration).

\subsubsection{Film dynamics at oscillations}

At oscillations, the liquid meniscus not only recedes but also advances over the already deposited film. Consider first the liquid meniscus advancing with a constant velocity $V_{a}$ over the film of a thickness $\delta_{0}$. This is a situation of e.g. the trailing meniscus of the Taylor bubble (Fig. 2a). One mentions that the interface shape in the transition zone from the film to the spherical meniscus part is wavy. Such a shape is often called "capillary shock". One can explain its origin with the following consideration. From the hydrodynamics equations, the fluid flow direction is that of $-\partial p_{l} / \partial x$. According to Eq. (26), this quantity is equal to $\sigma \partial K / \partial x$. For this reason, the monotonously increasing (from 0 to $\simeq r^{-1}$ ) curvature is incompatible with the negative flow direction created by the advancing liquid. A wavy shape (i.e. the sign variation of $\partial K / \partial x$ ) is needed to match them (Fig. 7b). The capillary shock is also observed during the meniscus oscillation (Fig. 10). 
The data on film dynamics can be obtained in experiments with forced oscillation, either with a single meniscus [95] (where the piston driving the oscillations is in contact with the liquid) or in oscillating plug experiments $[96,97]$ where the piston is in contact with the vapor phase. One needs to account for the plug mass loss caused by the film deposition [32] that leads to a difference of velocities of the meniscus and of the plug mass center. During oscillations, the meniscus velocity varies so the wavy film shape varies in time (cf. Fig. 7b). The local film

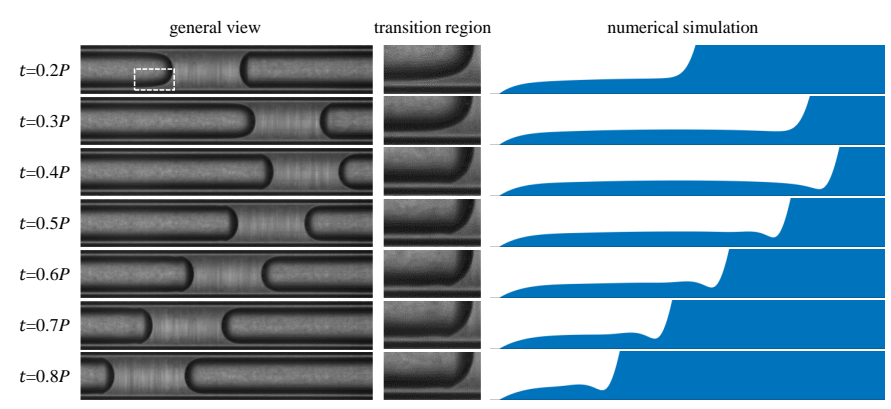

Figure 10: Liquid film shape during meniscus oscillation. Left column: experiment with n-pentane [96]. Middle column: zoomed transition region between the film and the meniscus (shown by white rectangle in the upper left image). Right column: numerical simulation in lubrication approximation with the pinned contact line [84]. $P$ is the oscillation period.

thickness follows the velocity of the meniscus. Therefore the thickness is smaller in the extreme meniscus positions (where the velocity is zero) and attains its maximum in the middle of the film as shown in Fig. 11.

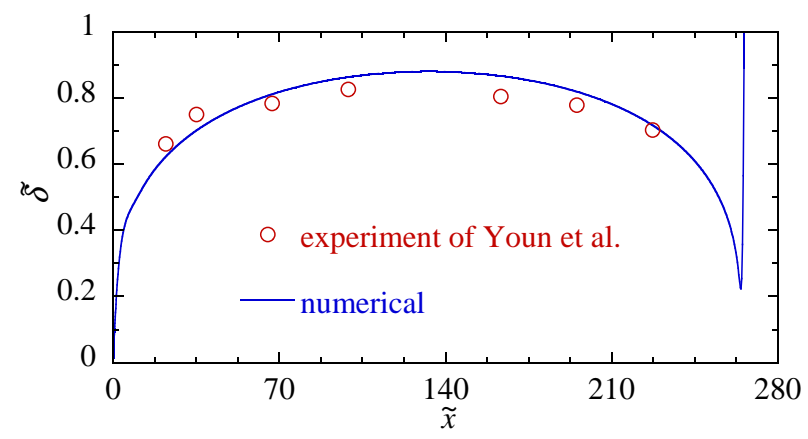

Figure 11: Comparison of the liquid film thickness [95] measured for ethanol at $2 \mathrm{~Hz}$ oscillation frequency with the corresponding numerical simulation [84]. Both variables are expressed in dimensionless units.

\subsubsection{Film length: contact line dynamics}

After consideration of the film shape near the meniscus, let us now discuss the vicinity of the contact line (CL) that can form in PHP (Fig. 2b). CL can move (advance or recede), which is a process required to be modeled theoretically to determine the film length. In this section, the classical problem with no phase change is described before addressing this latter effect in the following sections.

For the partial wetting conditions, the flow generated by the CL motion cannot be explained in the framework of conventional fluid mechanics approach where the Navier-Stokes equations are solved with the no-slip boundary condition $u_{x}(y=$
0) $=0$ at the wall, cf. Fig. 6 for the reference system. Indeed, CL belongs both to the liquid and to the solid and cannot move when the no-slip condition is applied. This phenomenon is frequently referred to as the CL hydrodynamic paradox. To solve it, an additional microscopic scale mechanism should be introduced into the theory.

The most common solution is an account of hydrodynamic slip length $l_{s}$, i.e. a replacement of the no-slip condition by the Navier slip condition at $y=0$ :

$$
u_{x}=l_{s} \frac{\partial u_{x}}{\partial y}
$$

Physically it means admitting a possibility for the liquid molecules to slip along the solid wall with a velocity proportional to the shear stress. Since the slip length is typically nm-scale [98], the right hand side differs from zero in a region where the shear is strong, which occurs only very close to CL.

In the case of complete wetting, the intermolecular (van der Waals) forces become dominant at small scales. It is convenient to describe them in terms of the disjoining pressure $\Pi(\delta)=H /\left(6 \pi \delta^{3}\right)$. The Hamaker constant $H$ is positive for complete wetting, and its typical value is $10^{-20}-10^{-19} \mathrm{~J}$. The disjoining pressure contributes to the Laplace equation (26),

$$
\Delta p^{i}=K \sigma+\Pi(\delta) .
$$

Due to a large value of $\Pi$ at small $\delta$, Eq. (29) describes a continuous wetting film connected to the liquid meniscus. The triple contact does not exist any more so the paradox does not occur. The film thickness scales on the Israelachvili length $l_{I}=$ $\sqrt{H /(6 \pi \sigma)} \sim 10 \mathrm{~nm}$. The same situation occurs in the pseudopartial wetting case where $H>0$ like for complete wetting but the equilibrium contact angle defined from the surface energies through the Young expression is non-zero (see Brochard-Wyart et al. [99] for the classification of wetting regimes).

In both mechanisms of singularity relaxation discussed above, the CL vicinity influenced by these microscopic effects is called microscopic region, which is tiny with respect to the macroscopic scale observable experimentally (Fig. 12). For the description of the CL motion, one needs to introduce an intermediate region (typically, in between $100 \mathrm{~nm}$ and $10 \mu \mathrm{m}$ from CL, Fig. 12b) governed by the interplay of the surface tension and viscous forces. Because of a strong scale difference, the hydrodynamic flow generated at the intermediate length scales is not directly impacted by the above microscopic effects. In fact they influence the intermediate region through a boundary condition imposed at a scale $\ell_{V}$ (called Voinov length) corresponding to the size of microscopic region. This boundary condition is the equality of the interface slope $\partial \delta / \partial x$ to $\theta_{V}$ (Voinov angle) defined at the upper boundary of the microregion. In the absence of evaporation (the evaporation case will be considered in sec. 2.3.5), $\theta_{V}$ is $\theta_{\text {micro }}$, which is the microscopic contact angle defined at a scale of a few $\mathrm{nm}$. Such an approach results in the Cox-Voinov law

$$
\left(\frac{\partial \delta}{\partial x}\right)^{3}=\theta_{V}^{3}-9 C a_{c l} \log \frac{x-X_{f}}{\ell_{V}}
$$




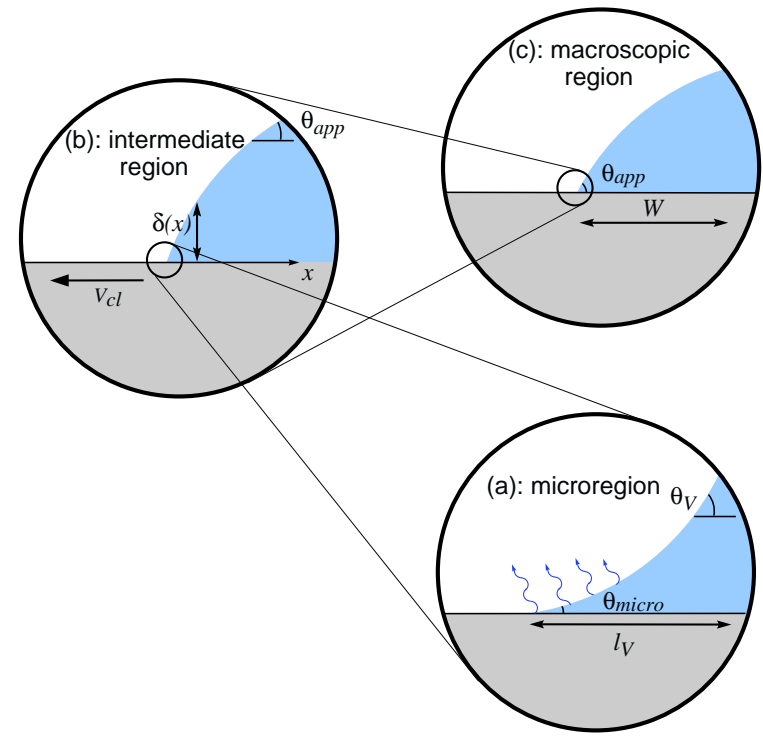

Figure 12: Hierarchy of length scales in the moving CL problem.

where $X_{f}$ is the CL coordinate. This law defines the slope in the intermediate region.

The aim of the CL problem is to find the interfacial shape in the macroscopic region, in particular, the apparent contact angle $\theta_{\text {app }}$ observed at the macroscopic scale. To determine it rigorously one needs to match the macroscopic and intermediate regions, or in other words, fit the known in advance macroscopic shape to the CL.

Let us outline this procedure on an example of the capillary dewetting phenomenon [89]. A water film (of $\sim 100 \mu \mathrm{m}$ thickness) that can be deposited with a knife onto a plastic (non wetting) substrate is unstable. If one creates a hole (dry area) in it, the hole begins to grow (Fig. 13). The liquid that covered pre-

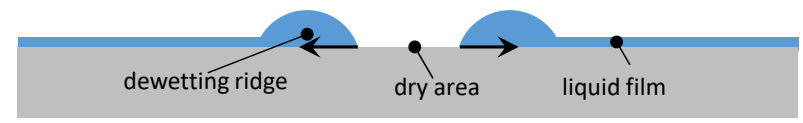

Figure 13: Dewetting ridge formation after the liquid film rupture on a nonwettable substrate.

viously the dry area is not able to flow inside the film because of the strong viscous shear characteristic of the thin films. For this reason the liquid is accumulated in a ridge surrounding the dry area. The ridge shape is nearly static and is thus circular.

Because of geometrical CL singularity, the microregion has a strong impact on the macroregion, in particular, on $\theta_{a p p}$. To find it numerically, one needs to resolve both the large and small scales. This is technically difficult because the meshing would need to be extremely fine so a large number of mesh cells would be necessary. It turns out however that the hydrodynamics can be solved separately in each of three regions. Thanks to the strong scale separation, the mathematical method of asymptotic matching can be used to assemble their solutions by performing a coupling at the boundaries of the regions. A remarkable outcome of such an approach consists in the fact that both $\ell_{V}$ and $\theta_{V}$ are independent of the macroregion geometry and are defined only by the microregion physics. As for the macroregion, the matching results in the expression

$$
\theta_{\text {app }}^{3}=\theta_{V}^{3}-9 C a_{c l} \log \frac{W}{\ell_{V}}
$$

where $W$ is the characteristic macroscopic length (Fig. 12c).

For the dewetting ridge geometry $[100,101], W=w / e$. For the case of the slip length theory, $\ell_{V}=\ell_{s} \equiv 3 l_{s} /\left(e \theta_{\text {micro }}\right)$. For the case of complete wetting, $\ell_{V}=\ell_{I}=0.63 l_{I} C a_{c l}^{-2 / 3}$ [102]. One mentions that all the terms in Eq. (31) are now defined, so $\theta_{\text {app }}$ can be determined.

It should be noted that at capillary dewetting, the CL velocity is completely defined by the contact angle $\theta_{V}$. According to the above theory that agrees with numerous experiments [103],

$$
C a_{c l}=\frac{\theta_{V}^{3}}{9}\left[\log \left(a C a_{c l}^{1 / 3} \frac{w^{2}}{\ell_{V} \delta}\right)\right]^{-1},
$$

where $a \approx 0.15$ is constant, and $\delta$ is the film thickness at the ridge-film junction ( $\delta=\delta_{0}$ for the adiabatic case of sec. 2.3.1, but is smaller when the evaporation is involved).

Two cases should be clearly distinguished. The first is the motion of CL over the dry wall (or over the nanoscale wetting film if the wetting is complete). It is a true CL motion considered here. The second case is a motion of liquid meniscus over the Taylor bubble film (sec. 2.3.2). In both cases the main effect on the liquid meniscus is often seen in terms of the apparent contact angle that depends on the CL velocity. The difference between these cases lies in the different film thickness. For the Taylor bubble film, the effect of velocity on the meniscus slope is relatively weak, and is defined mainly by the inertial effects affecting the meniscus curvature. At receding with low $R e$ and $W e$, when a film is deposited (sec. 2.3.1), the meniscus always exhibits $\theta_{a p p} \approx 0$. On the contrary, for the dry wall case, which can exist at $C a_{c l}<C a_{c r}, \theta_{a p p}$ is defined by Eq. (31) and the $\theta_{a p p}\left(C a_{c l}\right)$ dependence is much stronger.

\subsubsection{Liquid film profile at phase change}

One can consider now the phase change impact. It is simpler to start with the nearly flat film portion (Fig. 6). The contact line vicinity and the ridge will be addressed in the next section. First we evaluate the impact of phase change at constant liquid receding. In the absence of phase change and at constant velocity receding, a portion of the deposited film between the meniscus and the CL region is of constant thickness. This occurs because of the absence of liquid flow in the film: there is no pressure gradient inducing its motion. In the presence of phase change, the situation is different. While the flow along the film is still nearly absent, the film thins because of evaporations or thickens if condensation occurs. Consider the film evaporation. The thickness of the film portion deposited earlier becomes smaller with respect to that deposited later because of a larger evaporation time. The film interface is thus inclined during receding. 
To evaluate this effect, one can neglect the liquid flow in the film in Eq. (25) so the film thickness $\delta(x, t)$ changes because of phase change alone and the governing equation becomes

$$
\rho_{l} \frac{\partial \delta}{\partial t}=-J
$$

where the interfacial mass flux $J$ is assumed to be positive at evaporation. To obtain the film shape, consider the energy balance at the film surface. Its temperature $T^{i}$ is (nearly) equal to the saturation temperature $T_{\text {sat }}$ corresponding to the vapor pressure because the fluid is pure. By neglecting the heat flux into the vapor, one can write the interfacial energy balance as [80]

$$
\mathcal{L} J=\frac{\lambda_{l}\left(T_{w}-T^{i}\right)}{\delta}
$$

where the right hand side is the heat flux arriving from the liquid side of the interface. In the above expression, the stationary linear temperature distribution across the film is assumed. In the case of poorly conductive tube (plastic or glass), this approximation may be invalid, and the transient temperature distribution needs to be calculated [104]; this is however unnecessary in a more common case of a metal tube and a non-metal fluid.

The discussion below will be limited to the evaporation because of its importance (at film thinning, the phase change rate strongly increases).

The slope of evaporating film was introduced by Thome et al. [105] for the flow boiling description and applied later to the PHP modeling [60, 106-108]. By combining Eqs. (33) and (34) and by assuming a constant in time $\Delta T=T_{w}-T_{\text {sat }}(p)$ (i.e. both an isothermal evaporator wall due to its high heat conductivity and constant vapor pressure), one gets a closed form equation for $\delta$ that solves to [75]

$$
\delta(x, t)=\sqrt{\delta_{0}^{2}-2 \frac{\lambda_{l} \Delta T}{\mathcal{L} \rho_{l}}\left[t-t_{0}(x)\right]},
$$

where $t_{0}(x)<t$ is the time moment of the meniscus passing through the point $x$. The function $t_{0}(x)$ is obtained by inversion of the meniscus motion law $x=X_{m}\left(t=t_{0}\right)$. The thickness $\delta_{0}=\delta\left(t=t_{0}\right)$ is given by Eq. (27), which includes $V=\dot{X}_{m}\left(t_{0}\right)$ through $C a$. Fig. 14a is obtained by using Eq. (35) and by assuming the harmonic meniscus oscillation [75]. The slope agrees with the experimental measurements performed in the single-branch ethanol-filled PHP (Fig. 14b).

It is evident from the dimensional analysis that the film slope is controlled by the dimensionless parameter $\lambda_{l} \Delta T /\left(\mathcal{L} \rho_{l} V \delta_{0}\right)$ where $V$ is the receding velocity. The slope increases with the wall superheating and decreases with the velocity: for a larger velocity the time to create the slope is smaller.

This simple model results in the mass flux divergence $\sim \delta^{-1}$ near the CL which is of course non-physical but shows a necessity to account for the liquid flow on the microscopic scale. This is a subject of the next section.

\subsubsection{Contact line under phase change}

Consider now the phase change impact on the contact line vicinity, i.e. the microregion of Fig. 12a. First, we discuss

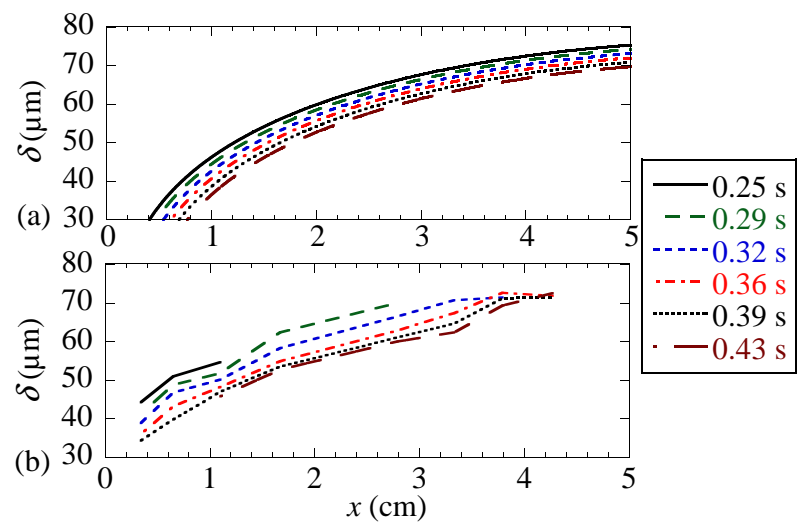

Figure 14: Film thinning caused by evaporation [75]: (a) Numerical result and (b) experimental measurement by interferometry.

the immobile CL (pinned on a defect, cf. sec. 2.3.1). The phase change combined with the CL motion (where the dewetting ridge of Fig. 6 appears) will be considered next.

The phase change induces a strong liquid-vapor interface curvature. There are two effects that cause it; the viscous pressure drop and the vapor recoil pressure. We first focus on the viscous effect Mathematically, it is described by the neglected in Eq. (33) second term of Eq. (25).

Consider a liquid wedge (Fig. 15). We assume a spatially

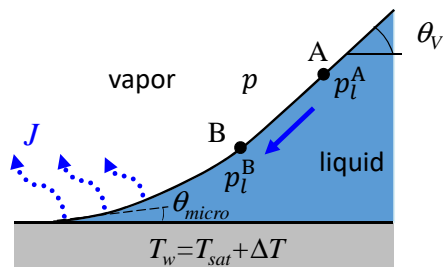

Figure 15: Liquid wedge at evaporation. The liquid flow is shown by the blue solid arrow.

isothermal wall of a temperature $T_{w}=T_{\text {sat }}+\Delta T$, which is usually a good approximation because of its high thermal conductivity. The liquid-vapor interface is nearly isothermal $\left(T^{i} \simeq\right.$ $\left.T_{\text {sat }}\right)$. Since the evaporation mass flux $J$ is given by Eq. (34), it increases strongly near the CL. This requires a liquid inflow to compensate the mass loss. Because of the viscous pressure drop, the liquid pressure varies along the liquid wedge. Consider two points at the interface: A and B. Point B is closer to $\mathrm{CL}$ so that the liquid flows from $\mathrm{A}$ to $\mathrm{B}$ and the liquid pressures in them satisfy the inequality $p_{l}^{\mathrm{A}}>p_{l}^{\mathrm{B}}$. The interfacial curvature $K$ is related to the pressure via Eq. (26). Since the vapor pressure $p$ is homogeneous, the local curvatures at these points satisfy the inverse inequality: $K_{l}^{\mathrm{B}}>K_{l}^{\mathrm{A}}$, which means that the curvature grows near the $\mathrm{CL}$, which in its turn, means that the interface is curved as outlined in Fig. 15. Namely, the interface slope $\theta_{V}$ far from the CL (which is the apparent contact angle observed macroscopically in such a system) turns out to be larger than its microscopic value $\theta_{\text {micro }}$. This effect was discovered by Potash and Wayner [109] for the geometry of the wedge with the attached nanometric film $\left(\theta_{\text {micro }}=0\right.$ case $)$ and studied by many other researchers. Among many others, one 
can mention the work of Stephan and Hammer [110] who have implemented this model for the heat transfer studies. For a long time, the disjoining pressure (necessary in this case to describe the thin film, cf. Eq. 29) was wrongly believed to be responsible for this phenomenon.

The second effect that can cause the interface slope increase is the vapor recoil force. When evaporation occurs, the fluid expands when changing the state from liquid to vapor. It means that on average, the evaporation-induced flow velocity in the vapor is much larger than in the liquid. To conserve the momentum at the interface, there is an additional pressure $p_{r}$ exercised toward the liquid. One can show [111] that

$$
p_{r}=J^{2}\left(\frac{1}{\rho_{v}}-\frac{1}{\rho_{l}}\right) .
$$

It needs to be included into the interfacial pressure balance (26) that becomes

$$
\sigma K=\Delta p^{i}+p_{r}
$$

Since the evaporation is strong near CL, $p_{r}$ and thus the curvature are both high there, which results in the interface bending as shown in Fig. 15. The apparent contact angle $\theta_{V}$ is thus larger than $\theta_{\text {micro }}[111]$.

The relative contribution of these two effects varies from one situation to another. Usually, the viscosity effect is stronger than that of the vapor recoil.

To determine the length scale at which a strong interface bending occurs, one needs to consider several microscopic effects. First of all, we consider the $T^{i}$ variation that occurs because of the interfacial pressure variation (Kelvin effect) and the interfacial thermal resistance [110]

$$
R^{i}=\frac{T_{s a t} \sqrt{2 \pi R_{v} T_{s a t}}\left(\rho_{l}-\rho_{v}\right)}{2 \mathcal{L}^{2} \rho_{l} \rho_{v}}
$$

that appears in a linearized version of the Schrage molecularkinetic theory [53]. The expression for $T^{i}$ accounting for both these effects reads [80]:

$$
T^{i}=T_{s a t}\left(1+\frac{\Delta p^{i}}{\mathcal{L} \rho_{l}}\right)+R^{i} J \mathcal{L} .
$$

One can see that because of the microscale effects, $T^{i} \neq$ $T_{\text {sat }}$. Each of these effects corresponds to a characteristic length [112]. For the Kelvin effect, it is

$$
\ell_{K}=\frac{\sqrt{3 \mu \lambda_{l} T_{\text {sat }}}}{\theta_{\text {micro }}^{2} \rho_{l} \mathcal{L}},
$$

and for the interface resistance, $\ell^{i}=\lambda_{l} R^{i}$. One recalls one more characteristic length, that of hydrodynamic slip $\ell_{s}$. It turns out that the largest of the microscopic characteristic lengths defines the microregion behavior (i.e. the value of $\ell_{V}$ ). Typically, it is $\ell_{s} \simeq 50-100 \mathrm{~nm}$ so we will use this value in what follows.

The interfacial slope induced by the phase change varies sharply near CL and finally saturates at a constant value $\theta_{V}$ at a scale $\sim \ell_{V}$. Note that this microregion behavior is different from the intermediate region logarithmic asymptotics (30) at large $x$.

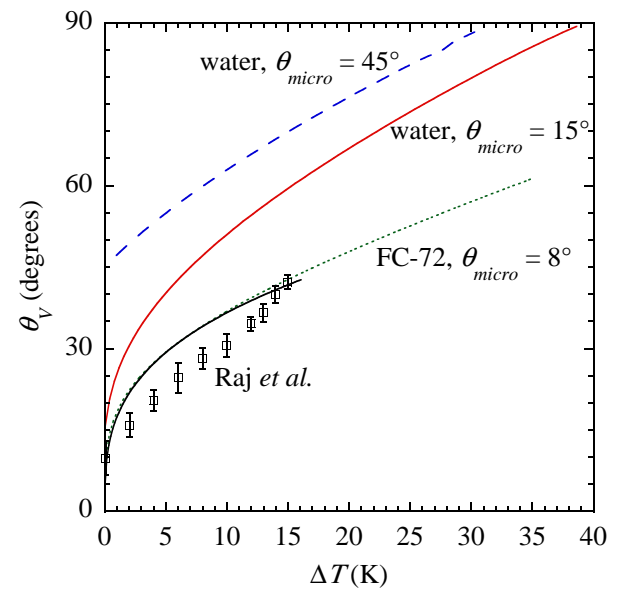

Figure 16: Dependencies of the apparent contact angle $\theta_{V}$ caused by evaporation in the CL vicinity on the wall superheating $\Delta T$ [113] calculated for water at $10 \mathrm{MPa}$ and two different $\theta_{\text {micro }}$ values. Curves calculated for FC-72 at $0.04 \mathrm{MPa}$ and $\theta_{\text {micro }}=8^{\circ}$ (dotted lines) are also shown for comparison with the theoretical results obtained for complete wetting by Raj et al. [114], black solid line. Their experimental data (where $\theta_{\text {micro }}=8^{\circ}$ ) are shown with squares.

Some typical dependencies of $\theta_{V}$ on the wall superheating are shown in Fig. 16. Note that the slope can grow from a small to very high value for a reasonable superheating.

Such a theory results not only in the $\theta_{V}$ but also in a dependence $J(x)$ for a given $\Delta T$. Janeček and Nikolayev [115] have shown that $J$ at the CL (at $\delta=0$ ) is finite. $J(\delta)$ can thus be integrated numerically [113] until a value $\ell_{V}$ beyond which the considered above dependence $J \sim \delta^{-1}$ is valid.

Some analytical solutions for $\theta_{V}$ can be obtained for the case where, among four microscale effects listed above, only the Kelvin effect is important. The asymptotic solution for small $\Delta T[115]$ reads

$$
\theta_{V}=\theta_{\text {micro }}+\frac{\pi \Delta T}{2 \sigma \theta_{\text {micro }}^{2}} \sqrt{\frac{3 \mu \lambda_{l}}{T_{\text {sat }}}},
$$

so $\theta_{V}$ is linear in $\Delta T$. For large $\Delta T$, the slope becomes independent of $\theta_{\text {micro }}[116]$ and varies weaker:

$$
\theta_{V} \simeq\left(\frac{\Delta T}{0.3 \sigma} \sqrt{\frac{3 \mu \lambda_{l}}{T_{\text {sat }}}}\right)^{1 / 3} .
$$

Recall that $\theta_{V}$ is the apparent contact angle observed in the presence of phase change when the CL is static.

Consider now the coupled phase change and the CL motion. As explained above, the effect of the fluid flow caused by phase change on the interface slope manifests itself in the microregion only (Fig. 12a): we saw that the characteristic scale $\ell_{V}$ of this phenomenon is extremely small. Therefore the phase change can be neglected inside the intermediate region ruled out mainly by the flow caused by the CL motion. For this reason, evaporation and CL motion are decoupled, and all the theory of sec. 2.3.3 can be applied to the case of CL with phase change. However the value $\theta_{V}$ caused by the phase change should now be used [116]. For practical purposes, one can reason that the system behaves as if the wetting properties were controlled with the substrate superheating. 


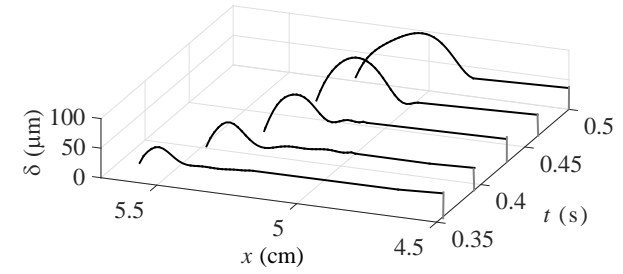

(a) The ridge dynamics measured by the optical grid deflection method.

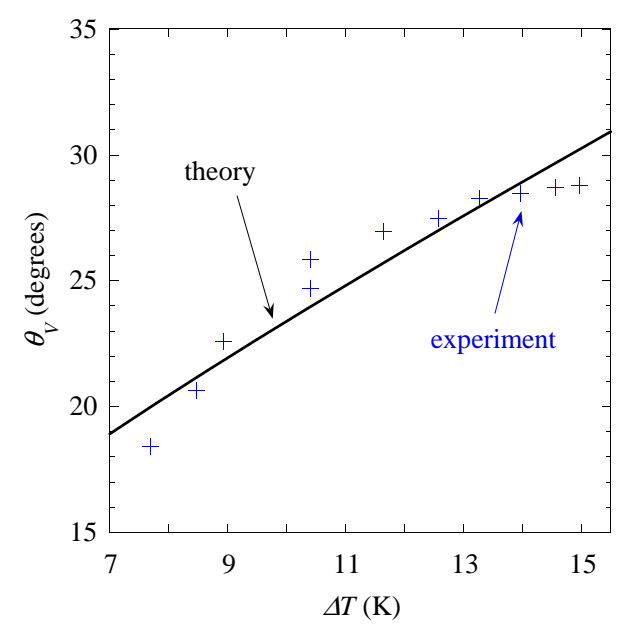

(b) The Voinov angle $\theta_{V}$ caused by evaporation versus wall superheating $\Delta T$.

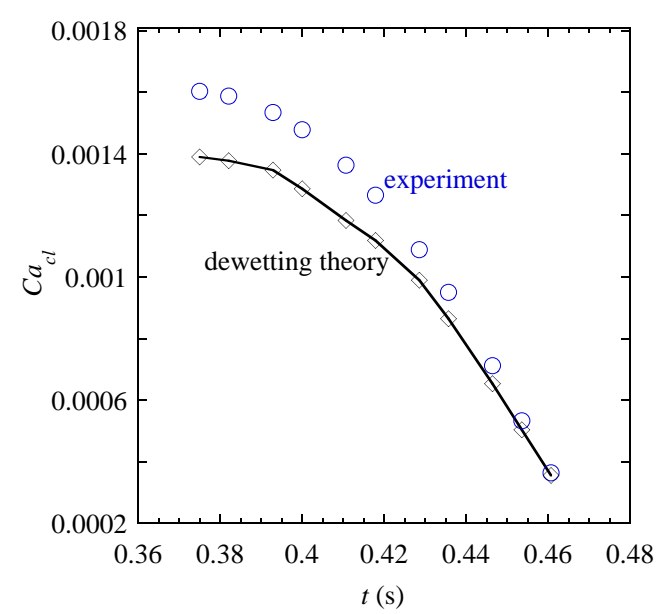

(c) Experimental data for $C a_{c l}$ compared to the values given by Eq. (32), i.e. by the dewetting theory [101] (at adiabatic conditions).

Figure 17: Evaporation-induced dewetting of the ethanol film on sapphire measured in the single-branch PHP [74].

Let us consider the implication of this theory for the PHP films. Once a liquid film is deposited by the moving liquid meniscus, its edge recedes because of two effects. First, the apparent contact angle becomes large because of evaporation, and the wall dewetting occurs, which causes the film length reduction. Second, the liquid in the CL vicinity vaporizes; CL recedes because of the evaporative mass loss. Let us discuss first the apparent contact angle effect. Experimental data demonstrating this phenomenon have been obtained by the ridge shape reconstruction [76] from the experimental images (cf. Fig. 9). The reconstructed axial film cross-section is presented in Fig. 17a. The ridge shape was fitted to a circle $[18,92]$. The slope at the circle intersection with the wall is the apparent contact angle $\theta_{\text {app }}$. Simultaneously, the CL velocity (i.e. $C a_{c l}$ ) and the ridge width $w$ were measured as functions of $\Delta T$. Then $\theta_{V}$ was calculated with Eq. (31). The only postulated value was $l_{s}=20 \mathrm{~nm}$; it was used for the $\ell_{V}=3 l_{s} /\left(e \theta_{\text {micro }}\right)$ estimation. The obtained in this way curve $\theta_{V}(\Delta T)$ can be compared to the microregion model [112] for $\theta_{V}$ computed by using the same $l_{s}$ value. Fig. 17b shows a good agreement. One mentions that the $\theta_{V}$ values can be high in spite of nearly zero $\theta_{\text {micro }}$ characteristic for ethanol on clean sapphire used in the experiment. Such high evaporation-caused $\theta_{V}$ values are at the origin of the wall dewetting.

The apparent contact angle was also measured as a function of $\Delta T$ in an earlier experiment [114] on drop evaporation with retraction, cf. Fig. 16. The discrepancy between the experimental and theoretical data can probably be attributed to the neglect of the CL velocity (i.e. of the second term in Eq. 31).

This phenomenon differs from a ridge formation during static meniscus evaporation in micron-sized capillaries at high heat fluxes [117]. Such a ridge appears because of the interfacial shear created by the vapor outflow. This effect is expected to be negligible in the millimeter-sized PHP capillary because of a much lower vapor velocity.

Let us consider now the relative contribution of evaporative mass loss at the CL. Fig. 17c presents the experimental data of the CL receding velocity together with the result of Eq. (32), which describes only the first (dewetting) contribution [74]. One can see that CL recedes mainly because of the contact angle growth via dewetting; the evaporative mass loss is responsible only for $14 \%$ of the CL velocity.

\subsubsection{Film modeling in existing PHP simulations}

One can distinguish several kinds of the liquid film 1D models that appeared historically in the PHP modeling community. As mentioned in sec. 2.2.1, Shafii et al. [31] and their followers [62, 68-70, 118-121] to cite some of them, described the heat exchange in the vapor domain with Eq. (20), which is equivalent to the heat exchange wall-vapor with no liquid film. In conjunction with the ideal gas EOS this ("superheated vapor") model was quite well studied. It results typically in small-amplitude oscillations [122].

Holley and Faghri [46] and their followers [34, 65, 123125] adopt a model where the liquid film covers completely the wall within the vapor bubbles. The film thickness is assumed constant and fixed a priori. The film volume in each bubble is used to determine the void fraction, that, in its turn, is used to calculate the heat exchange coefficient $U_{f}$ according to a flow boiling correlation. The heat exchange in the vapor domain of the length $L_{v}$ is computed as (cf. Eq. 20)

$$
\dot{Q}_{v}=U_{f} \wp L_{v}\left[T_{w}-T_{s a t}(p)\right] .
$$

The main advantage of such an approach is its simplicity. However, it cannot describe the dry spot formation in the evaporator 
which are known to play an important role in PHP, for example to describe the intermittent oscillation regime or the PHP dryout. The application of this model to the single and multibranch PHP is discussed in sections 3.1 and 4.1, respectively.

Das et al. [122] have suggested a minimal complexity, "film evaporation-condensation" (FEC) model capable to describe the dry spots. It is based on the lumped meniscus geometry (Fig. 18) proposed earlier by Dobson [63] (who however used the superheated vapor equations), where the films of constant thickness but variable length were considered. It is formulated here for the simplest case of the single branch PHP with the imposed temperatures in both the condenser and the evaporator.

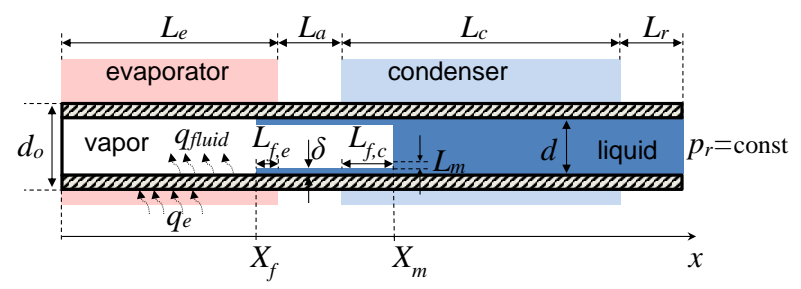

Figure 18: The lumped meniscus representation of the single branch PHP and the coordinate system.

The phase change rate $\dot{m}$ is obtained by integration of the mass evaporation flux (34) over the film area and the contributions from evaporation in the evaporator and condensation in the condenser are written separately $(k=e$ in evaporator and $k=c$ in the condenser):

$$
\dot{m}_{f, k}=\frac{U_{f}}{\mathcal{L}} \wp L_{f, k}\left[T_{k}-T_{s a t}(p)\right],
$$

where $L_{f, k}$ is a film portion belonging to the section $k=e, c$ (Fig. 18).

The film heat transfer coefficient $U_{f} \sim \lambda_{l} / \delta$. A coefficient of proportionality of the order one (the film form factor) is introduced to account for the film slope (sec. 2.3.4) so the film mass flux can be written in Eq. (43) as if the axial film profile was rectangular.

The film length is assumed to vary because of two factors, the phase change and the meniscus motion over the film. During the meniscus advancing, the film length reduces and viceversa. This can be described by the CL dynamics equation [126]

$$
V_{f}= \begin{cases}V_{m} & \text { if } X_{f}=X_{m}, V_{m}<0, \\ \dot{m}_{f, e} /\left(\rho_{l} S_{f}\right) & \text { otherwise, }\end{cases}
$$

where $V_{f}=\dot{X}_{f}$ is the film edge velocity and $S_{f} \simeq \wp \delta$ is the film cross-section area. The first line of Eq. (43) is introduced to make $X_{f}$ coincide with the meniscus position $X_{m}$ during the meniscus advancing over the dry area when the film length becomes zero. In all other cases, the film edge recedes because of the film evaporation contribution defined by the evaporation part $\dot{m}_{f, e}$ of the total film mass change rate $\dot{m}_{f}$. The film can cover parts of both evaporator and condenser at the same time. $V_{f}$ is then assumed to be controlled only by the evaporator part $L_{f, e}$ of the film. This is necessary to avoid a nonphysical film extension. Imagine a situation where $X_{f}$ situates in evaporator but $X_{m}$ is so deep in the condenser that the film condensation in the condenser overcomes the film evaporation in the evaporator. In reality, the CL receding occurs in such a situation. If one used the full film contribution $\dot{m}_{f}$ in Eq. (43)instead of $\dot{m}_{f, e}, V_{f}$ would be negative, which is nonphysical. The other part of the mass flux $\dot{m}_{f, c}$ (condensed onto the film) is added to the liquid plug to conserve the total fluid mass.

A deficiency of the FEC model is a necessity to choose a fixed a priori film thickness $\delta$. To simulate the regimes of continuous oscillations, this issue is solved by the following iteration algorithm $[73,127]$ : a plausible $\delta$ is chosen, then the root mean square plug velocity is used to calculate the dimensionless numbers $\mathrm{Ca}, \mathrm{Re}, \mathrm{We}$ which in their turn are used in the expression (27) to calculate a new $\delta$. The simulations are repeated until a convergence is achieved.

FEC model was used by several other teams to describe the 2-branch (U-turn) PHP [128] and a multi-branch PHP [33, 72, 108]. Its implementation for the single and multi-branch PHP (code CASCO) is discussed in sections 3.2 and 4.2, respectively. It is a "minimal complexity" model that can describe the multi-branch PHP, but not necessarily the best from the physical point of view, because implicitly assumes an instant end-toend hydrodynamic flow along the film, which is nonphysical. Indeed, within such a model, evaporation at any film portion causes the instant film edge receding, which assumes implicitly a hydrodynamic flow along the film (which is prohibited by the hydrodynamic equations because of strong viscous shear, cf. sec. 2.3).

In an extension of the FEC model proposed by Rao et al. [104], the CL contribution to the evaporation flux was introduced together with the thinning of the film of homogeneous thickness. Rao et al. [55] have managed to estimate experimentally the separate contributions of the film $U_{f}$ and CL heat transfer (described by the heat transfer coefficient $U_{c l}$ ). They have used this data to account for the time variation of both the spatially homogeneous film thickness $\delta=\delta(t)$ and the film length. To describe the CL dynamics, instead of Eq. (43), they introduce an equation

$$
V_{f}= \begin{cases}V_{m} & \text { if } X_{f}=X_{m}, V_{m}<0, \\ \dot{m}_{f, c l, e} /\left[\rho_{l \wp} \delta(t)\right] & \text { otherwise, }\end{cases}
$$

where the evaporation rate at $\mathrm{CL}$ in evaporator is

$$
\dot{m}_{f, c l, e}= \begin{cases}\frac{U_{c l}}{\mathcal{L}} \wp \delta(t)\left[T_{e}-T_{\text {sat }}(p)\right] & \text { if } X_{f} \leq L_{e}, \\ 0 & \text { otherwise. }\end{cases}
$$

The film thickness time variation is defined by the equation

$$
\dot{\delta}=-\frac{\dot{m}_{f, e}}{\rho_{l} \wp L_{f, e}},
$$

where $\dot{m}_{f, e}$ is given by Eq. (42) where $U_{f} \sim \lambda_{l} / \delta(t) ; L_{f, e}=$ $L_{f, e}(t)$ is related to $X_{f}$ via Eq. (62) below. Eq. (46) is completely equivalent to Eqs. (33-34). For this reason, this model presents a better alternative to the original FEC model. In addition, according to Eq. (44), the CL position changes because 
of the local CL evaporation and not because of the film overall evaporation postulated in FEC model. Therefore, a liquid flow along the film is absent, in agreement with the thin film theory discussed in sec. 2.3.1. While this approach is more accurate than FEC model, it requires a knowledge of an additional parameter $\left(U_{c l}\right)$ that should be determined experimentally.

By using this model, one can estimate the validity range of the FEC model. It is justified when the film thickness variation during an oscillation period is not strong, i.e. $2 \pi \dot{\delta} / \omega_{0} \ll \delta$, where $\dot{\delta}$ is given by Eq. (46).

An obvious limitation of the FEC model is its inability to describe accurately the film shape and thus the mass exchange that may impact the PHP dynamics. For multi-branch PHP modeling, d'Entremont and Thome [60], Nemati and Shafii [129] used the wedge film evaporation model with the variable film thickness $\delta=\delta(x, t)$ defined by the local evaporation (sec. 2.3.4) The film was assumed continuous and was meshed according to the wall mesh. At film deposition, $\delta=\delta_{0}$ is defined by the receding meniscus velocity via a correlation similar to Eq. (27) thus providing an initial condition. After deposition, the film thickness at a wall point $x$ varies following Eqs. (33-34), where $T_{w}=T_{w}(x, t)$, and $T^{i}=T_{\text {sat }}$ can vary in time. This means that the film flow is neglected, just like in the FEC model. A minimum thickness $\delta_{\min }$ of the order of surface roughness was imposed to cut off the divergence of evaporation rate in Eq. (34). When the film attains this minimum thickness at a point, its evaporation stops. Such a model is capable of capturing the film slope effect described in sec. 2.3.4 but requires solving an extra partial differential equation and keeping track of $\delta(x, t)$ on the wall mesh. For these reasons it is more resource consuming than the FEC model. The dependence of this model on $\delta_{\text {min }}$ is expected to be strong because it cutoffs the otherwise divergent $J \sim \delta^{-1}$, so it is a major control parameter.

In addition to the film thinning discussed above, Senjaya and Inoue [106] introduced the 1D modeling of a flow along the film. The liquid pressure distribution along the film is introduced. It is coupled to the inhomogeneous pressure calculation in the vapor phase. Consequently, the required computer resources (time, memory) are much larger than for the FEC model. The tangential shear created by the vapor flow is accounted for. For millimetric channels used in PHP and small velocities observed in it, this effect should however be negligible.

The film flow equations of Senjaya and Inoue are based on phenomenological considerations rather than on the hydrodynamic thin film theory. In particular, they assimilated the film to a tube of the equivalent cross-section with an inflow proportional to the difference $\left(r-\delta_{0}\right)$. In reality, the film inflow is controlled by the interfacial curvature gradient rather than the film cross-section (sec. 2.3.1. To show the difference, one can consider the flat film case. According to Eqs. (25-26), the flow is nonexistent, unlike the flow described by equations of Senjaya and Inoue. As in the wedge film evaporation model, introduction of $\delta_{\min }$ was necessary. Some results of this model are discussed in sec. 4.3.

As a conclusion, all existing liquid film PHP models involve at least one control phenomenological parameter that needs to be adjusted to achieve an agreement with the experimental data. A truly physical model accounting for the film flow and CL motion dynamics presented above is yet to be developed. It should be based on the micro-scale modeling as discussed in sec. 2.3.5 to describe correctly the film length influenced by the dewetting effect.

\subsection{Interaction between bubble or plug pairs}

In a 1D PHP simulation, two neighboring bubbles or two neighboring plugs are considered to be independent. This is an approximation as in reality such an interaction can exist for two bubbles separated by a short plug, or, inversely, for two plugs separated by a short bubble because of the hydrodynamic effects. Such phenomena are yet to be studied, but their impact is not expected to be strong. A strong interaction occurs when a bubble (or a plug) between two neighboring plugs (or bubbles) appears or disappears.

First, consider the vapor bubble disappearance. It occurs when a moving liquid plug overtakes another plug. The vapor pressure grows and fast condensation occurs. Finally, the bubble disappears and a new composite plug forms. In a 1D computation, to prevent the negative bubble length one usually introduces a minimum bubble length $L_{\text {min }} \simeq 10 \mu \mathrm{m}$ at which it is considered to disappear [127]. The composite plug mass is a sum of the masses of parent plugs and the bubble of the length $L_{\text {min }}$. The plug velocity is determined from the momentum conservation [32].

The vapor bubble generation is an extremely important feature to obtain the stable PHP operation. In horizontal orientation, without the bubble generation, the oscillation finally stops whatever is the film model [106, 127]. The bubble generation was first introduced by Senjaya and Inoue [67]. Their model describes a generation of "tube-size" bubbles, i.e. bubbles of a small length that occupy all the tube cross-section (except the film thickness). This is the only option in a 1D model. The bubbles of a fixed length $L_{n u c l}$ are assumed to nucleate at several nucleation sites (typically, one per branch) under two simultaneous conditions justified by their experimental observations. The first condition is conventional

$$
T_{w}(x)-T_{\text {sat }}(p)>\Delta T_{\text {nucl }}
$$

and means that the wall superheating at the nucleation point $x$ exceeds a nucleation barrier $\Delta T_{n u c l} \sim 5-20^{\circ}$, which is a typical for the onset of nucleate boiling. The pressure $p_{\text {nucl }}$ of the newly nucleated bubble is equal to the liquid pressure at the nucleation site location. The liquid pressure is assumed to vary linearly along the plug, so the pressure is defined by the linear interpolation between the pressures of the $i$-th (preceding) bubble and the next bubble (with respect to the $x$-axis directed along the PHP channel),

$$
p_{\text {nucl }}=p_{i}+\left(p_{\text {next }, i}-p_{i}\right) \frac{x-X_{i}^{r}}{L_{l, i}}
$$

where $X_{i}^{r}$ is the right meniscus of the $i$-th bubble.

The second condition of nucleation of Senjaya and Inoue is a limitation on the liquid plug velocity, $\left|V_{i}\right| \leq 0.2 \mathrm{~m} / \mathrm{s}$. This 
value is based on their experimental observations in the transparent evaporator of a horizontal PHP. The tube-size bubble are observed to appear as a result of growth and coalescence of small diameter bubbles, which nucleate and grow on the hot wall. If the plug velocity is small enough, the small bubbles grow and coalesce to form tube-size bubbles during plug residence in the evaporator. If the plug velocity is large, the small bubbles easily detach from the wall and do not grow nor coalesce. The tube size bubble is not formed and the small bubbles disappear when the plug enters the condenser. No velocity criterion was defined in the subsequent studies however. In the model, liquid films are assumed to be continuous at the bubble formation. The authors do not discuss the minimal distance $L_{n u c l, m i n}$ from the end of the plug at which the bubbles are allowed to nucleate. Apparently, it was introduced: when a plug approaches the nucleation site inside the evaporator, the pressure is the smallest at the leading plug meniscus so the criterion (47) is satisfied right at the meniscus. However the nucleation should occur inside the plug so $L_{n u c l, m i n}$ should be necessarily introduced. Physically, it corresponds to the time (multiplied by the meniscus velocity) needed to form a thermal boundary layer needed for the bubble growth.

Another particularity of the model is an assumption of a faster growth of the newly nucleated bubbles: the evaporation film heat exchange coefficient $U_{f}$ was imposed to be ten time larger than for other bubbles. For smaller multipliers, the liquid plug oscillations were small and sinusoidal like in the Shafii et al. [31] simulation; it did not attain the nucleation site and the bubble generation did not occur.

Ethanol-filled PHP with no adiabatic section was simulated. With no bubble generation, only small amplitude sinusoidal oscillations appeared that ended up with a dryout. With the bubble generation, the amplitude of oscillation was large and stable. The same feature was observed later [127]. Senjaya and Inoue [130] performed an analysis of bubble generation parameters. In particular, they showed a strong dependence (increase) of the PHP performance on the number of nucleation sites.

The deficiencies of the above model (in particular, a large number of numerical criteria specific for given regime) have been overcome in subsequent simulation approaches [33, 73, 127] where the nucleation was allowed in any point of PHP where the criterion (47) holds. The difference between these approaches is minor. Nekrashevych and Nikolayev [73, 127] consider that $\Delta T_{\text {nucl }}$ is a constant quantity defined by the wall surface state. Daimaru et al. [33] fixed instead the bubble inception radius $r_{0}=1 \mu \mathrm{m}$ and calculated $\Delta T_{n u c l}$ with the Hsu formula reflecting the Kelvin effect [53],

$$
\Delta T_{\text {nucl }}=\frac{2 \sigma T_{\text {sat }}}{r_{0} \rho_{v} \mathcal{L}}
$$

In a recent approach [131], even a more complicated correlation (accounting for the case where the thermal boundary layer thickness is comparable to the surface roughness) is used. It is not clear if it impacts the simulation results.

Another interaction event that should be implemented in a PHP simulation is the liquid plug disappearance [127]. It typically occurs when a long plug penetrates into a dry and hot evaporator section. A bubble is nucleated and a short plug of the length $L_{\text {nucl,min }}$ is pushed into a hot dried tube portion. During its advancing, the plug deposits a liquid film but does not absorb the film ahead because the evaporator is dry. Such a film deposition consumes the whole plug mass. In a simulation, the plug disappearance is assumed when it becomes smaller than a deletion threshold $L_{\text {min }}$.

A study of the bubble dynamics following their generation has been performed by Nekrashevych and Nikolayev [127]. If the wall is hot enough, many bubbles can be generated one after another at the same hot spot so "bubble trains" are formed until the hot spot is cooled down, cf. Fig. 19. Right after gen-

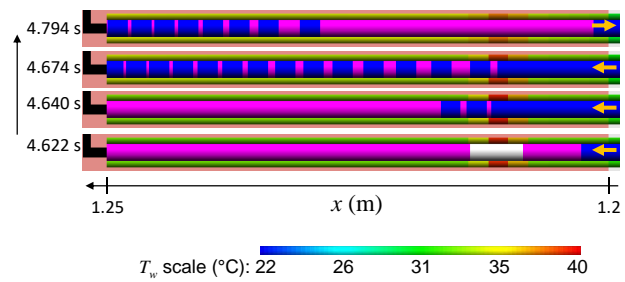

Figure 19: An example of multiple bubble generation in the evaporator of the multi-branch PHP filled with FC-72 calculated with CASCO. Arrows show the direction of motion of the rightmost liquid plug [127]. The blue color indicates the liquid, violet and gray, the bubbles; violet are the film areas while the gray zones are dry.

eration, the velocity of a bubble train coincides with that of the parent plug. The growth of a bubble in the evaporator causes compression of neighboring bubbles and the direction of plug motion can be inverted (cf. Fig. 19 for $t=4.794 \mathrm{~s}$ ). Such an inversion means an oscillation. This shows the mechanism of oscillation sustainment by bubble generation. As a result of the bubble generation, the hot (nucleation) spot is cooled down and the generation stops.

A liquid plug can appear inside a bubble (via the bubble break-up) either because of the dewetting ridge growth until it forms a liquid bridge [86] or because of the inertial liquid film instabilities [132]. This phenomenon is yet to be studied for inclusion into the PHP simulations.

\section{1D modeling of the single branch PHP}

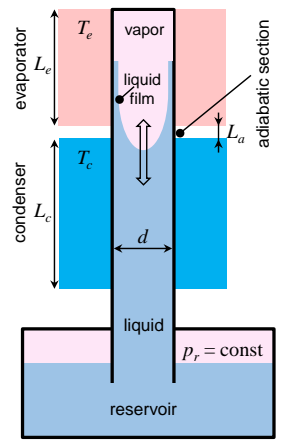

Figure 20: Typical schematic configuration of the vertical single branch PHP.

To evaluate the validity of different PHP models, it is necessary to test them first on simple PHP geometries, to obtain 
more general, if possible analytical, results. In addition, the local experimental data can be obtained for such geometries so a detailed comparison with the modeling is possible. With this knowledge, one can adjust phenomenological parameters to use them in simulations of the multi-branch PHP. We consider here the simplest, single-branch PHP, see Fig. 20. It is a straight capillary with one sealed heated end, which thus forms an evaporator. The middle part of the capillary is cooled and is thus condenser. The other end of capillary is connected to a reservoir maintained at a constant pressure $p_{r}$. A single gas bubble is confined between the sealed end and a liquid plug. Once a necessary temperature difference between evaporator and condenser is established, the liquid meniscus starts oscillating. One can see that such a system represents a kind of a "unit cell" (a unique bubble-plug couple) for a more complex multi-branch PHP. Several experiments have been conducted in vertical [55, 74, 75, 104, 133, 134] and horizontal [54, 122, 125] orientations.

From the experimental point of view, the single branch PHP is much easier to study than the multi-branch PHP for several reasons. First, the vapor location is known in advance so the temperature sensors can be put inside it without being wetted intermittently, which is important to provide reliable data (cf. sec. 2.2.1). Second, a smaller view field is required to film the whole extension of meniscus motion so more details about it can be found. Third, the frequency of oscillations can be controlled by choosing the PHP tube length and the reservoir pressure $p_{r}$, which is useful to select a required data acquisition rate.

The first question one can ask about the PHP is "why it oscillates?". One can explain the functioning mechanism of the single branch PHP as follows. When the meniscus situates in the evaporator, evaporation occurs. The vapor expands and the meniscus is pushed into the condenser. The condensation occurs, and the vapor bubble contracts so the meniscus returns back into evaporator. The liquid plug inertia provides a phase shift between the pressure and meniscus position, which is well known (Barkhausen) criterion of self-sustained oscillations.

The liquid plug momentum equation stems from Eq. (9) with $p_{\text {next }}=p_{r}$,

$$
\frac{\mathrm{d}\left(V_{m} m_{l}\right)}{\mathrm{d} t}=\left(p-p_{r}\right) S-F+G,
$$

where

$$
\dot{X}_{m}=V,
$$

and the liquid mass should include a part of the liquid in the reservoir that is brought to motion during oscillation.

We consider first the simplest version of the model where the conduction via the PHP tube is neglected and the temperatures of the inner tube walls are imposed to be equal to $T_{c}$ and $T_{e}$ in condenser and evaporator, respectively. The heat exchange with the tube is absent in the adiabatic section. These assumptions are applied in many models [31, 63, 122]. In this case the heat exchange inside the liquid does not impact the plug dynamics and can be solved a posteriori to evaluate the global heat exchange. In the next stage, we introduce the heat conduction through the tube walls and caused by it smooth wall temperature variation, see sec. 3.2.2 below.

At the PHP start-up, a small random fluctuation can either cause oscillations or on the contrary relax, so PHP comes to some stationary non-oscillating state of dynamic equilibrium (that we will call simply equilibrium for the sake of brevity). One needs thus to find such state and see if it is stable or unstable with respect to a small fluctuation. A stable state can be found experimentally and means the absence of oscillation while an unstable mode corresponds to the oscillation start-up. We describe now two models most commonly used for the PHP simulation and analyze the stability of the single-branch PHP described within each of them.

\section{1. "Superheated vapor" modeling}

We begin the analysis of the reduced version of the model of [31], which is based on the heat exchange in the vapor defined by Eq. (20). As mentioned in sec. 2.3.6, it is equivalent to the hypothesis of the dry walls within the vapor bubbles. The vapor energy balance is written for the fixed vapor mass $m$ so (22) reduces to $m c_{v v} \dot{T}=\dot{Q}_{v}-p \dot{\Omega}$ that can be rewritten in the form

$$
m c_{v v} \dot{T}=U_{v} \wp\left[L_{v, e}\left(T_{e}-T\right)+L_{v, c}\left(T_{c}-T\right)\right]-p S V_{m}
$$

The lengths of the vapor in contact with the evaporator and the condenser are defined by the expressions

$$
\begin{aligned}
L_{v, e} & = \begin{cases}X_{m}, & X_{m} \leq L_{e}, \\
L_{e}, & \text { otherwise },\end{cases} \\
L_{v, c} & = \begin{cases}0, & X_{m}<L_{e}+L_{a}, \\
X_{m}-\left(L_{e}+L_{a}\right), & L_{e}+L_{a} \leq X_{m}<L_{e}+L_{a}+L_{c}, \\
L_{c}, & \text { otherwise. }\end{cases}
\end{aligned}
$$

The system is governed by the set of differential Eqs. (50-52) and the EOS (21). The viscous friction and gravity are neglected for simplicity.

Such a simplified formulation that keeps the main features of the original model has an advantage of being analyzable analytically [122] via the conventional linear stability analysis to find the conditions of PHP start-up. First one finds equilibrium states (denoted by the overbar hereafter) by zeroing the time derivatives. Then a small perturbation of such a state is introduced. One linearizes the set of equations with respect to the perturbations and finds a condition for their growth (i.e. when the equilibrium is unstable).

For such a model, there is an infinity of equilibrium states. At equilibrium, the analysis [122] shows that the meniscus can situate in any section (evaporator, adiabatic, or condenser). The equilibrium meniscus positions in evaporator and adiabatic sections are stable for any system parameters. This is a nonphysical result. Indeed, when an experimentalist wants to start-up a single-branch PHP, the meniscus is brought precisely to the evaporator or adiabatic section [75, 134].

For equilibrium meniscus positions located in the condenser (i.e. for $L_{e}+L_{a} \leq \bar{X}_{m}<L_{e}+L_{a}+L_{c}$ ), the instability occurs 
when [122]

$$
\bar{T}-T_{c}>(\gamma-1) \bar{T}\left(1-\frac{L_{a}}{\bar{X}_{m}}\right),
$$

where the equilibrium vapor temperature $\bar{T}$ is determined by the following expression obtained by cancelling the derivatives in Eq. (52),

$$
\bar{T}=\frac{L_{e} T_{e}+\left(\bar{X}_{m}-L_{e}-L_{a}\right) T_{c}}{\bar{X}_{m}-L_{a}}
$$

Since $\bar{T}$ grows with $T_{e}$, the condition (55) is roughly equivalent to the statement that the difference $T_{e}-T_{c}$ must exceed a threshold. We have thus found theoretically a well-known empirical PHP start-up criterion [13]. A numerical solution of Eqs. $(50,51,52)$ indeed exhibits oscillations that develop under condition (55). The model however shows a nonphysical behavior. At many combinations of parameters, the oscillations occur while the meniscus remains inside the condenser during the whole oscillation cycle [122], so no evaporation is possible. Generally, the amplitude of oscillations is quite small with respect to the evaporator length.

Let us now consider now the oscillation start-up within a formulation that corresponds to the liquid films covering entirely the tube inside the vapor bubbles. In this case the vapor mass varies due to the film evaporation-condensation. The phase change rate is introduced via Eq. (42). Like in the approach of Shafii et al. [31], the time variation of vapor mass is

$$
\dot{m}=\frac{U_{f}}{\mathcal{L}} \wp\left[L_{v, e}\left(T_{e}-T\right)+L_{v, c}\left(T_{c}-T\right)\right] .
$$

The vapor energy balance (22) should now be used (with $\dot{Q}_{v}=0$ because the dry area is absent). Such an equation can be solved analytically as discussed in sec. 2.2.2 and leads to Eq. (24). Since the pressure is homogeneous at equilibrium, $\bar{p}=p_{r}$, and the coefficient in Eq. (24) can be determined explicitly:

$$
p=p_{r}\left(\frac{T}{\bar{T}}\right)^{\frac{\gamma}{\gamma-1}} \text {. }
$$

By using EOS (21), one can express $T$ through $m$ and $X_{m}$ :

$$
T=\bar{T}\left(\frac{\bar{T} R_{v} m}{p_{r} S x_{m}}\right)^{\gamma-1} .
$$

With the substitution of two latter equations, Eqs. (50, 51, 57) form a closed system, the stability of which can now be analyzed. One mentions however that by expressing $m$ as a function of $T$ and $X_{m}$ with Eq. (59) and taking its derivative, one obtains exactly Eq. (52) with $U_{v}$ replaced by $U_{f} R_{v} T / \mathcal{L}$. In other words, the start-up analysis shows the equivalence of the superheated vapor model with no films, and with the continuous films surrounding a vapor bubble. Both formulations can thus be addressed as the superheated vapor model. As shown above, the formulation with no films behaves in a nonphysical way. To conclude this part, the model formulated initially by Shafii et al. [31] and used by many research groups (including a recent theory [125]) is not fully adequate, at least for the single branch PHP, and for this reason is hardly reliable for advanced multibranch PHP modeling. A more advanced model needs thus to be used.

\subsection{FEC modeling}

The FEC model has been introduced in sec. 2.3.6. In the following, we consider separately two model statements, first for constant wall temperatures in the evaporator and condenser sections, and next for the smooth temperature variation along the wall due to its heat conduction.

The natural frequency of oscillations is an important parameter, especially at the start-up, where the oscillation amplitude can be small. The horizontal single branch PHP is characterized by the eigenfrequency of the inviscid liquid plug [122] of the average length $\bar{L}_{l}$,

$$
\omega_{0}=\sqrt{\frac{\gamma p_{r}}{\rho_{l} \bar{L}_{l} \bar{L}_{v}}},
$$

and depends only weakly on the phase change intensity. When the PHP tube is vertical, the gravity term appears [55]:

$$
\omega_{0}=\sqrt{\frac{\gamma p_{r}}{\rho_{l} \bar{L}_{l} \bar{L}_{v}}+\frac{g}{\bar{L}_{l}}} .
$$

\subsubsection{PHP startup model for imposed temperatures in evapo- rator and condenser}

Imagine that the tube temperatures are $T_{e}$ and $T_{c}$ in evaporator and condenser, respectively, and both these values are constant due to high wall heat capacity. Instead of Eq. (42), one obtains the following expression for the film phase change rate [122] $\dot{m}_{f}=\dot{m}_{f, e}+\dot{m}_{f, c}$ where both terms are defined with Eq. (42) with

$$
\begin{aligned}
& L_{f, c}= \begin{cases}0, & \text { if } X_{m} \leq L_{e}, \\
X_{m}-L_{e}, & \text { if } L_{e}<X_{m} \leq L_{e}+L_{c}, \\
L_{c}, & \text { otherwise. }\end{cases} \\
& L_{f, e}= \begin{cases}X_{m}-X_{f}, & \text { if } X_{f}<X_{m}<L_{e}, \\
0, & \text { if } X_{m} \leq X_{f}, \\
L_{e}-X_{f}, & \text { if } X_{m} \geq L_{e},\end{cases}
\end{aligned}
$$

where $L_{a}=0$ is assumed. The total vapor change rate

$$
\dot{m}=\dot{m}_{f}+\dot{m}_{m},
$$

includes the meniscus contribution that exists even in the absence of the film. More precisely, it corresponds to the contribution of the CL vicinity (cf. sec. 2.3.5) of the size $L_{m}$ (Fig. 18) over which the heat exchange coefficient is denoted $U_{m}$,

$$
\dot{m}_{m}=\frac{U_{m} L_{m} \wp}{\mathcal{L}}\left[T_{w}\left(X_{m}\right)-T_{s a t}\right] .
$$

The problem includes now five differential equations for the variables $V_{m}, T, m, X_{m}, X_{f}$ (instead of three in the superheated vapor model). First two are the mechanical momentum and the vapor energy equations (50) and (22). One cannot use its reduced form (24) because dry areas are allowed and one needs to account for $\dot{Q}_{v}$ given by Eq. (20). Other three differential equations of the model are Eqs. (43, 51, 63). The EOS (21) closes the problem. 
For the simplest case $L_{a}=0$, one easily finds that the only stationary state is possible when the meniscus situates at the boundary between the evaporator and the condenser.

It is difficult to define the start-up conditions in a general case because the parameters are too many and the diagram would be multidimensional. It is however possible to obtain it in the approximation of small temperature difference $T_{e}-T_{c}$, more specifically, in the approximation of the small dimensionless quantities

$$
\begin{aligned}
\alpha_{c} & =\frac{U_{f}\left[T_{\text {sat }}\left(p_{r}\right)-T_{c}\right]}{\omega_{0} \rho_{l} \mathcal{L} \delta}, \\
\alpha_{e} & =\frac{U_{f}\left[T_{e}-T_{\text {sat }}\left(p_{r}\right)\right]}{\omega_{0} \rho_{l} \mathcal{L} \delta},
\end{aligned}
$$

where $\omega_{0}$ is given by Eqs. (60). The parameters $\alpha_{c}$ and $\alpha_{e}$ characterize the film condensation in the condenser and film evaporation in the evaporator, respectively. The parameter

$$
\Gamma=\frac{U_{v}(\gamma-1)}{\omega_{0} c_{p v} \rho_{l} \delta}
$$

characterizes the heat exchange of the wall with the vapor. The

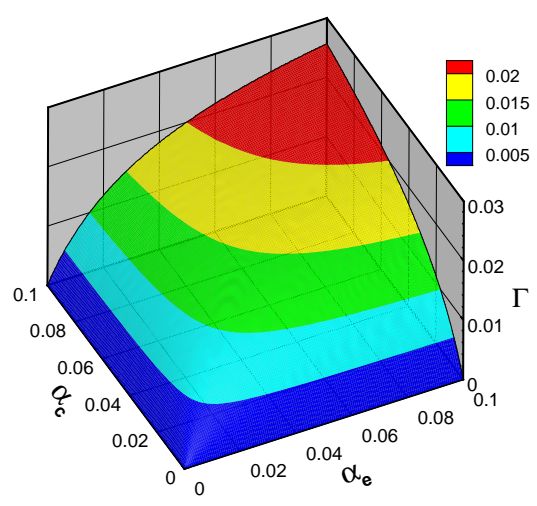

Figure 21: Instability boundary for the single branch PHP with imposed temperatures in evaporator and condenser in the parameter space $\left(\alpha_{c}, \alpha_{e}, \Gamma\right)$ [135]. The boundary is presented as a surface which is the dependence of $\Gamma$ on $\alpha_{c}$ and $\alpha_{e}$. The oscillations can develop for the points below the surface.

threshold criterion can be expressed as $\Gamma=f\left(\alpha_{c}, \alpha_{e}\right)$. The surface corresponding to this function is shown in Fig. 21. The system is unstable (i.e. the PHP starts up) for the points below the surface (i.e. for $\Gamma<f\left(\alpha_{c}, \alpha_{e}\right)$ ) and stable for the points above the surface.

Several general conclusions can be drawn from this figure. First, both condensation and evaporation are necessary for oscillations, which corresponds to the inequality

$$
T_{c}<T_{\text {sat }}\left(p_{r}\right)<T_{e} .
$$

Indeed, if either $\alpha_{c}$ or $\alpha_{e}$ are zero, no instability is possible. The increase of $\alpha_{c}$ or $\alpha_{e}$ favors the instability. If the system is characterized by a point $\left(\alpha_{c}, \alpha_{e}, \Gamma\right)$ located above the surface in Fig. 21 (i.e. in the stable region), an appropriate increase of $\alpha_{c}$ or $\alpha_{e}$ (achieved by increasing $T_{e}$ or decreasing $T_{c}$ ) brings the system to the instability. This qualitatively agrees with experiments: the threshold value of $T_{e}-T_{c}$ is one of a few well established features of the PHP start-up [13].
Another consequence of Fig. 21 is that the heat exchange of the dry evaporator walls with the vapor (characterized by $U_{v}$ ) hinders the oscillations and can be thus considered as a channel of energy dissipation. Indeed, an increase of $\Gamma \sim U_{v}$ can bring the system above the surface in Fig. 21, where the system is stable. This feature can be easily understood. Since the heat exchange between the dry evaporator walls and the gas does not contribute to the evaporation/condensation mass exchange (which is the moving force of oscillations), the corresponding energy is lost, which means that $\Gamma$ characterizes the energy dissipation. This means that, in general, the fluids with low vapor heat conductivity are advantageous for PHP.

Since the film thickness is an independent parameter of this model, one can consider the dependence of the threshold on it. On one hand, an increase of $\delta$ leads to the decrease of $\alpha_{c}$ and $\alpha_{e}$ (cf. Eqs. (65) and thus hinders the instability. On the other, it leads to the decrease of $\Gamma$ (cf. Eq. 66)), which favors the instability. This means that in general there is a range of $\delta$, for which the oscillations develop. Beyond this range the system is stable. This conclusion agrees with the simulations of the multi-branch PHP [32].

\subsubsection{Impact of the thermal fluid-solid coupling on the PHP startup}

Consider now the impact of the wall heat conduction that cause a spatial variation of the tube wall temperature. Instead of (42), the film mass exchange reads

$$
\dot{m}_{f}=\frac{U_{f} \wp}{\mathcal{L}} \int_{X_{f}}^{X_{m}}\left[T_{w}(x)-T_{s a t}\right] \mathrm{d} x .
$$

To define the evaporative part of the total mass flux (to replace Eq. 42), one needs to introduce a notion of the effective evaporator as a part of the wall with $T_{w}>T_{\text {sat }}$ (over which evaporation occurs). Accordingly, the effective condenser is the remaining film part. The $T_{w}(x)$ variation is now monotonous (cf. Fig. 22 below); the effective evaporator edge $X_{e}$ can be determined from the equation

$$
T_{w}\left(X_{e}\right)=T_{\text {sat }} .
$$

The evaporative part of the total mass flux $\dot{m}_{f, e}$ (needed to define the CL dynamics with Eq. 43) can now be defined as

$$
\dot{m}_{f, e}=\frac{U_{f} \wp}{\mathcal{L}} \int_{X_{f}}^{X_{e}}\left[T_{w}(x)-T_{s a t}\right] \mathrm{d} x .
$$

Such a model formulation provides the liquid films covering the tube walls in the effective condenser (a section where $T_{w}(x)<$ $T_{\text {sat }}$ ), which corresponds to the condenser and adiabatic sections for the imposed $T_{e}$ and $T_{c}$ case.

Eq. (14) should be solved to determine $T_{w}(x)$. The adiabatic boundary condition is usually a good approximation at the sealed end of the tube,

$$
\left.\frac{\partial T_{w}}{\partial x}\right|_{x=0}=0 .
$$

The fluid-wall coupling complicates strongly the problem because one needs to solve more complex equations and the 
number of parameters is larger. However its account is of importance because it changes substantially the system dynamics and the start-up criteria [126].

First, consider the equilibrium states. Unlike the imposed temperatures case, there is a unique equilibrium state (denoted by overbar) for arbitrary $L_{a}$. It is given by the meniscus position $\bar{X}_{m}$, vapor temperature $\bar{T}$ and the wall temperature distribution $\bar{T}_{w}$ (see Fig. 21). $\bar{T}_{w}$ varies with $x$ because of the axial heat conduction and radial heat exchange with the vapor. Its characteristic length scale is $\ell_{d}=\sqrt{S_{w} \lambda_{w} /\left(\wp U_{v}\right)}$.

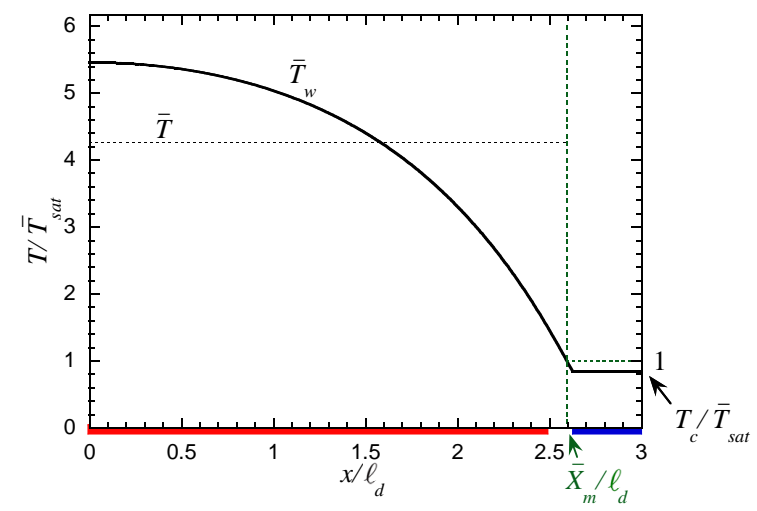

Figure 22: An example [126] of equilibrium temperature distribution $\bar{T}_{w}(x)$ along the PHP tube (solid curve). The equilibrium vapor temperature $\bar{T}$ is shown inside the vapor with a dotted line. The temperatures are made dimensionless with $\bar{T}_{\text {sat }}=T_{\text {sat }}\left(p_{r}\right)$. The locations of evaporator and condenser are shown with the red and blue bars, respectively.

It is evident that, at equilibrium, the meniscus position $\bar{X}_{m}$ coincides with the edge $\bar{X}_{e}$ of effective evaporator, so the liquid film is absent. Any deviation from it would cause the net mass exchange and thus the meniscus motion. By definition of the effective evaporator, the wall temperature at this point coincides with $\bar{T}_{s a t}>T_{c}$, which is apparent in Fig. 22. This means that $\bar{X}_{m}$ cannot belong to the condenser. In this particular case, $\bar{X}_{m}$ situates in the adiabatic section (i.e. $\bar{X}_{m}>L_{e}$ ).

It turns out that the relevant parameter for the start-up criterion is the gradient of $\bar{T}_{w}$ at $x=\bar{X}_{m}$, i.e. a slope of the curve in Fig. 22 at $x=\bar{X}_{m}$. In the most common case $\bar{X}_{m}>L_{e}$, this quantity is defined by the evaporator power,

$$
-\left.\frac{\mathrm{d} \bar{T}_{w}}{\mathrm{~d} x}\right|_{x=\bar{X}_{m}}=\frac{P_{e}}{S_{w} \lambda_{w}} .
$$

For the case $\bar{X}_{m}<L_{e}$ (the meniscus belongs to the evaporator), the r.h.s. of Eq. (72) should be multiplied by the factor $\bar{X}_{m} / L_{e}$. Such a formula may seem counter-intuitive because the temperature gradient is independent of the condenser temperature. It becomes however easier to accept if one recalls that this temperature difference appears due to the power injection into evaporator because the initial PHP temperature is that of condenser.

An analytical instability criterion [126] can be obtained within two hypotheses. First, it is the approximation of massive tube so at small deviations from equilibrium, the tube thermal response is slow and the temperature distribution in it does not deviate from the equilibrium distribution (Fig. 22) during the startup. Second, one assumes a small evaporator power so the averaging approximation is applicable. One can then obtain an analytical expression for the start-up threshold [126] that, in dimensional variables, reads

$$
\begin{aligned}
\frac{P_{e}}{S_{w} \lambda_{w}}=\left(\left.\frac{\bar{X}_{m}}{\gamma p_{r}} \frac{\mathrm{d} \bar{p}}{\mathrm{~d} T}\right|_{s a t}\right)^{-1} & \\
& +\frac{U_{v} \mathcal{L}}{U_{m} L_{m} R_{v}} \frac{(\gamma-1)^{2}}{\gamma}+\frac{8 v p_{r} \mathcal{L}}{d \bar{T} U_{m} L_{m} R_{v}} .
\end{aligned}
$$

One can see that the $P_{e}$ threshold is defined by three additive terms. The first term presents the inverse efficiency of phase change expressed in terms of the slope of saturation curve (cf. Fig. 4). This means that the high slope of saturation curve favors the oscillation start-up. This agrees with the experimental findings $[136,137]$. There are many other PHP parameters (like e.g. $T_{c}$ ) that influence implicitly the threshold through this term because $\bar{X}_{m}$ depends on them (this concerns also the quantity $\bar{T}$ in the third term). Two other terms correspond to different dissipation channels that need to be overcome to create self-sustained oscillations. The second term is related to the energy loss (in a sense that it is lost for creation of oscillations) appearing because of the tube-vapor heat exchange as already discussed in sec. 3.2.1. The last term is the contribution of viscous dissipation. Usually this term is expected to be smaller than the previous; however its contribution is inversely proportional to the tube diameter and may become crucial for thin capillaries (micro PHP).

An important feature of the start-up threshold is its independence of the film thickness, which suggests that the criterion (73) is independent of the particular film model. Instead, this formula shows the crucial role of the mass exchange at the meniscus for the oscillation start-up. One recalls that the meniscus mass exchange is provided by the meniscus part closest to the wall. In the absence of the film, this is the CL vicinity. The meniscus mass exchange is described by the group $U_{m} L_{m} / \mathcal{L}$. Once it is put to zero, the threshold becomes infinite so the oscillation start-up is impossible. This feature is completely different from the imposed temperatures case (sec. 3.2.1) where the PHP can start oscillations due to the film exchange alone. One can see that the account of the tube heat conduction is very important for the correct understanding of the PHP start-up.

A comparison of Eqs. $(72,73)$ shows a strong dependence of the threshold evaporator power on the tube parameters: it is (roughly) proportional to the product $S_{w} \lambda_{w}$. This occurs because a larger heat conductivity requires a larger power to produce the same temperature gradient. One can reasonably guess that this result applies to the multi-branch PHP too.

The threshold depends only weakly on condenser temperature or adiabatic section length. Such a situation is characteristic to an open PHP (that connected to a reservoir with the imposed pressure) for which the relevant parameter is the saturation temperature corresponding to the reservoir pressure rather than the condenser temperature. The role of $T_{c}$ in the open PHP is to bring meniscus close to the condenser, i.e. to increase the bubble length $\bar{X}_{m}$ thus lowering the oscillation threshold. 
To conclude this section, the PHP starts oscillating when the evaporator power exceeds energy dissipation and the phase change is efficient enough. Two causes of energy dissipation have been evidenced: the heat losses due to the vapor-tube heat exchange and the liquid viscous dissipation. The FEC model gives the start-up threshold that can be easily interpreted and, conceptually, is more realistic than the threshold given by the superheated vapor model.

\subsection{Experimental validation of the single branch PHP models}

Comparison to the experiments is most often performed for the steady regime that can be defined as a regime where the system state averaged over the oscillation period remains constant in time. In this regime, the frequency of oscillations is close to the natural frequency given by Eqs. (60).

In the dynamic simulations (Fig. 23), one clearly observes a strong effect of the vapor compression: the maximal vapor pressure is attained when $X_{m}$ is (nearly) the smallest, i.e. for the leftmost in Fig. 18 position of the meniscus.

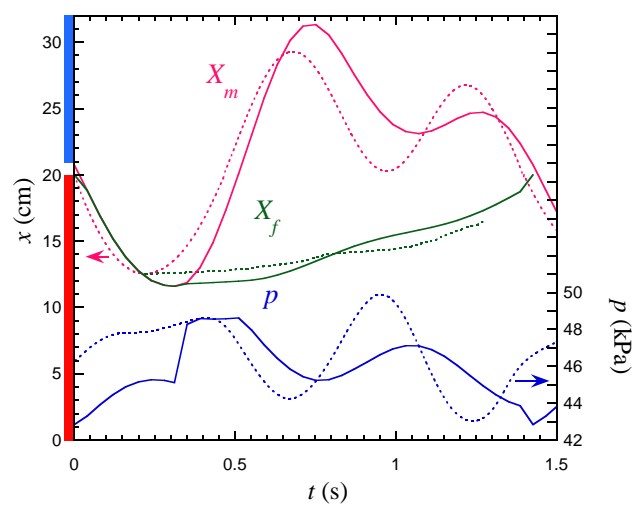

Figure 23: Comparison of experimental data and simulation performed with the extended FEC model, Eqs. (44-46), for meniscus evolution, CL evolution and vapor pressure for FC-72 with $T_{e}=37.5^{\circ} \mathrm{C}, T_{c}=16^{\circ} \mathrm{C}, p_{r}=50 \mathrm{kPa}$ for one period of oscillations (adapted from [104]). The evolution of $X_{m}, X_{f}$ and $p$ is shown with red, green, and blue colors, respectively. The theoretical and experimental data are shown with solid and dotted curves, respectively. The evaporator and the condenser are marked with the red and blue bars, respectively.

Two oscillation regimes have been predicted [122]. In the first regime, as observed experimentally [54, 75, 125, 134], the meniscus oscillations are quasi-sinusoidal (Fig. 5a). In the second regime, the amplitudes of two consecutive meniscus strokes strongly differ [55, 104, 133] so the meniscus penetrates into evaporator each second stroke and the period of oscillation is doubled (Fig. 23). The quasi-sinusoidal regime appears when the film phase exchange is strong so the film length (corresponding to the evaporated film mass in FEC model) changes considerably over an oscillation period, the oscillation amplitude is large and the liquid plug inertia is strong. The period doubling appears when the film mass exchange is not strong and the system needs time to accumulate evaporated mass. As the period of oscillations is imposed by the plug inertia and vapor thermodynamics, the system uses several oscillation periods for this. Rao et al. [55, 133] have shown experimentally that a phase shift can exist between $X_{m}$ and $p$ : $p$ maximum occurs when the vapor volume increases from its minimum (Fig. 23), i.e. during receding of the liquid meniscus. This shift is explained by the contribution of film evaporation (i.e. vapor mass increase) that causes the vapor pressure increase according to Eq. (21). Indeed, when the liquid meniscus recedes, the film area in evaporator grows and evaporation increases.

The massive heat spreaders are commonly used in PHP prototypes to reduce the temperature fluctuations. On one hand, a thermal spreader provides a thermal inertia that smoothes the temporal fluctuations but increases the time of the PHP transients (in particular, the start-up time). On the other hand, a spreader thermally shunts the tube so the spatial fluctuations along the evaporator are reduced. Generally, to describe a spreader, one needs to write a separate heat diffusion equation for it and introduce its thermal interaction with the tube. An example of such a model is given by Gully et al. [54] and the steady oscillations simulated within such a model are shown in Fig. 5b.

One can see that the FEC model provides an excellent qualitative description and fair quantitative agreement with the experiment. Inclusion of the CL effects can improve the model quality.

\section{1D modeling of the multi-branch PHP}

\section{1. "Superheated vapor" modeling}
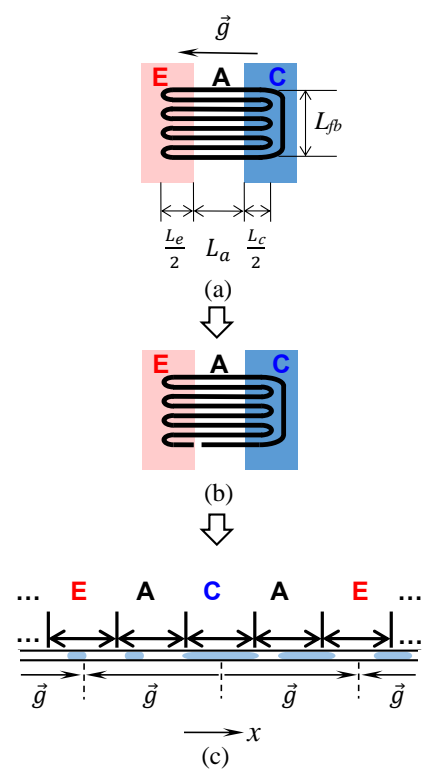

Figure 24: (a) Closed loop PHP of a simple geometry in the bottom heated orientation with $N_{p}=4$. (b,c) Geometrical transformations of the tube. (b) Unlooping (c) Unbending and projection to the $x$ axis. The splitting to branches is shown together with the gravity direction in each branch.

Shafii et al. [31] pioneered the 1D multi-branch PHP modeling. Both the vapor bubbles and the liquid plugs occupy all the tube cross-section. The PHP meander is represented as a straight tube with periodic boundary conditions; the PHP periods containing evaporator, adiabatic, condenser and again adiabatic sections follow each other sequentially (Fig. 24). The 
number of such periods $N_{p}$ is the main geometrical parameter (called also the number of PHP turns). In the work of Shafii et al., it is equal to three. The feedback section (a vertical section in Fig. 24) is not simulated $\left(L_{f b}=0\right)$. We denote hereafter both a bubble and the plug to the right of it (with respect to the $x$ axis direction) by the same index $i=0, \ldots, M-1$. The left end of the plug is thus $X_{i}^{r}$ and the right is $X_{\text {next }, i}^{l}$, where $X_{\text {next }, i}^{l}=X_{i+1}^{l}$ unless $i=M-1$, in which case $X_{n e x t, i}^{l}=X_{0}^{l}$.

The total number $M$ of bubble-plug couples may change in time because of the plug coalescence. The equations of motion are the sequential versions of the superheated vapor model equations $(9,51,57)$

$$
\begin{aligned}
& \frac{\mathrm{d}}{\mathrm{d} t}\left(m_{l, i} V_{i}\right)=\left(p_{i}-p_{\text {next }, i}\right) S-F_{i}+G_{i}, \\
& \dot{m}_{i}=\frac{\wp}{\mathcal{L}}\left[U_{e} L_{v, e, i}\left(T_{e}-T_{i}\right)+U_{c} L_{v, c, i}\left(T_{c}-T_{i}\right)\right], \\
& \dot{X}_{i}^{r}=\dot{X}_{\text {next }, i}^{l}=V_{i},
\end{aligned}
$$

where $V_{i}$ is the $i$-th plug center of mass velocity and $F_{i}$ is given by Eq. (8). Two more equations corresponding to $(21,24)$ have to be added to describe the vapor thermodynamics:

$$
\begin{aligned}
& p_{i} S L_{v, i}=m_{i} R_{v} T_{i}, \\
& p_{i}=p_{0, i}\left(\frac{T_{i}}{T_{0, i}}\right)^{\frac{\gamma}{\gamma-1}},
\end{aligned}
$$

where the index 0 identifies the initial bubble state.

To provide the mass conservation in the tube, they subtract from a plug a half of fluid mass evaporated to two neighboring bubbles:

$$
\dot{m}_{l, i}=-\frac{1}{2}\left(\dot{m}_{i}+\dot{m}_{i+1}\right)
$$

One notices that Eq. (76) lacks consistency as the plug volume varies but both the liquid density and the liquid plug length are constant. Such an approximation remains however reasonable provided the evaporated mass over an oscillation period remains small for each plug (this is not true for small plugs). The meniscus positions are corrected after each time step. If a meniscus position $X_{i}^{s}$ (with $s=r$ or $l$ ) becomes larger than $L_{t}$, it is replaced with $X_{i}^{s}-L_{t}$, where $L_{t}$ is the total length of the PHP tube. When it becomes smaller than zero, it is replaced with $X_{i}^{s}+L_{t}$.

Such a model results in a steady oscillation regime after a transient during which the number of liquid plugs drops from an initially imposed large number to $N_{p}$. During oscillations, the liquid remains in the condenser bends, while the vapor persisted in the evaporator bends. The oscillations of menisci are periodical and nearly sinusoidal, with the amplitude $\approx 0.15 L_{e}$. In other words, the multi-branch PHP acts like an assembly of oscillating masses coupled through the vapor pressure (springs). We call this regime small amplitude oscillations as the liquid plugs never transit through the evaporator bends.

Sakulchangsatjatai et al. [138] compared the overall heat transfer within the model of Shafii et al. with the existing experimental data and concluded that the agreement was satisfactory. However they did not mention their simulation parame- ters, in particular, the most important adjustable heat transfer coefficients $U_{e}$ and $U_{c}$.

Holley and Faghri [46] introduced the tube heat conduction and its thermal coupling with the fluid (see sec. 3.2.2) and changed the film model (see sec. 2.3.6). This a very important milestone. However the vapor energy equation they used was incorrect, see sec. 2.2.2. Mameli et al. [34] introduced into their code the pressure losses in the turns (see sec. 2.1.1).

Peng et al. [120] implemented the superheated vapor model for the eight-turn PHP. For $U_{e}$ and $U_{c}$, they took the flow boiling heat transfer coefficient that is generally much larger than the film heat transfer coefficient. The justification for this choice is not given. They simulated three different fluids: ammonia, water and acetone. The oscillation regime was mainly chaotic with the bottom heated mode and small amplitude oscillations were observed in the horizontal PHP orientation. One of their aims was to verify if the circulation regime is described by their model. The circulation regime corresponds to a nonzero average velocity of all liquid plugs. This regime is desirable in PHP because the heat transfer in it is believed to be maximal. When choosing the circulation initial conditions for ammonia they indeed observed the continuous circulation. They however simulated a short period of time where the average values of their system variables varied and the steady regime (cf. sec. 3.3 for its definition) was hardly attained. The circulation regime was not possible in the horizontal PHP orientation, which corresponds to experimental observations.

Daimaru et al. [124] implemented the model version with the continuous liquid films (no dry areas) for the multi-branch PHP, just like Dilawar and Pattamatta [123] did for the twobranch (U-turn) PHP. The fluid-tube thermal interaction was implemented and the heat flux was homogeneously distributed along the tube in the evaporator section. The heat exchange boundary conditions were implemented in the condenser section, i.e. Eq. (19) was applied there. The PHP start-up was not smooth, i.e. the evaporator temperature exhibited a peak before dropping when the oscillations began. They were small amplitude.

Yoon and Kim [139] implemented the superheated vapor model for the five-turn ethanol-filled PHP in the bottom-heated vertical orientation. They obtained and analyzed the similar, small-amplitude oscillations, which they also observed experimentally. By testing this model for a 30-branch PHP Bae et al. [108] have shown by comparing with the experiment that the superheated vapor model can be applied in the vertical position. However it does not provide oscillations for horizontal orientation for any reasonable value of the heat transfer coefficients $U_{e}$ and $U_{c}$. Fig. 25a demonstrates that the evaporator sections dry out and the oscillation stops after a short transient, which is contrary to their experimental observations.

\subsection{FEC approach for multi-branch PHP}

Nikolayev [32] has generalized the FEC model (sec. 3.2.1) to the multi-branch PHP with arbitrary $L_{a}$ for the imposed temperatures case. In addition, several simulation algorithm improvements have been proposed to make the simulation more 
robust. In the preceding works, an explicit (Euler) time marching scheme was used. This method is known to be numerically unstable, exhibiting artificial oscillations that can be confused with the true physical oscillations [122]. The 4th order Runge-Kutta method known for its numerical stability has been introduced as a core solver. In this method (as in any method more complex than Euler's), the right hand sides of differential equations need to be evaluated several times at each time step. This requires however a special management of bubble and plug disappearance as it can become necessary at one of such evaluations to avoid the negative bubble and plug lengths. Such system changes (called events hereafter) lead to a change in the number of variables and for this reason require a restart of calculation of the time step after detection of an event and its settling. To optimize the code architecture, it was also proposed to keep all the information on the current state of a bubbleplug couple within a unique data structure and use the dynamic memory allocation (reserving-liberating the computer memory at runtime). This is more convenient than reserving statically (at the compilation stage) a fixed memory amount and face a risk of its overflow because an extra bubble is generated.

The management of PHP looping was optimized because of a large number of coordinate variables per bubble (film edge coordinates). Within the approach of Shafii et al., they would need to be corrected at each time step to make them belong to the interval $\left(0, L_{t}\right)$. Instead, all the bubble-plug "train" is allowed to displace freely along an infinite axis. The periodicity condition now reads $X_{n e x t, M}^{l}=X_{0}^{l}+L_{t}$. No coordinate corrections are needed in such an approach, which accelerates the execution. To find out the position of a point inside the PHP tube, one needs to reduce its coordinate $x$ by finding a remainder of division by $L_{t}$.

Unlike the superheated vapor modeling, the FEC model targets the large oscillation amplitudes observed experimentally. This requires a thorough management of the fluid mass conservation to be able to simulate long evolution without deterioration of the FR value. In particular, instead of using Eqs. (76), one needs to account for the plug mass change because of the film deposition/absorption, so the velocities of menisci are determined from a set of equations

$$
\begin{aligned}
V_{i} & =\frac{1}{2}\left(\dot{X}_{i}^{r}+\dot{X}_{i, \text { next }}^{l}\right), \\
m_{l, i} & =\rho_{l} S\left(X_{i, \text { next }}^{l}-X_{i}^{r}\right) .
\end{aligned}
$$

They result in the velocities of menisci different from the plug velocity $V_{i}$.

Nekrashevych and Nikolayev [127] have further developed the FEC model to include the effects of wall heat conduction and bubble generation. The heat power was homogeneously injected into the tube walls within the evaporator section while the temperature of the internal tube walls was imposed in the condenser section. Some other features were added, like the feedback section description and the pressure loss at the bends (see sec. 2.1.1). To implement the FEC model, notions of the effective evaporator and condenser had to be generalized to the case of multi-branch PHP where the temperature distribution along a bubble can be non-monotonous. Because of this, several dry areas per bubble and, accordingly, several film pairs can appear. This is necessary to manage a possible appearance of long bubbles covering two or more evaporator sections. For the bubble $i$, the left and right film edges are denoted $X_{f, i}^{l, k}$ and $X_{f, i}^{r, k}$ for the $k$-th pair, cf. Fig. 26. The effective evaporator is defined as a part of the wall, which is either dry or along which $T_{w}>T_{\text {sat }, i} \equiv T_{\text {sat }}\left(p_{i}\right)$. The other bubble-covered part is the effective condenser. The variable number of effective evaporators is denoted $N_{e, i}$ and their left and right borders as $X_{e, i}^{l, k}$ and $X_{e, i}^{r, k}$, respectively. From such a formulation, the following inequalities are satisfied: $X_{i}^{l} \leq X_{e, i}^{l, 1} \leq X_{f, i}^{l, 1} \leq X_{f, i}^{r, 1} \leq X_{e, i}^{r, 1} \leq \cdots \leq X_{e, i}^{l, N_{e, i}} \leq$ $X_{f, i}^{l, N_{e, i}} \leq X_{f, i}^{r, N_{e, i}} \leq X_{e, i}^{r, N_{e, i}} \leq X_{i}^{r}$.

Similarly to the single-branch PHP model of sec. 3.2.2, Introduction of the effective evaporators $\left(X_{e, i}^{l, k}\right.$ and $\left.X_{e, i}^{r, k}\right)$ is necessary for separate calculation of the evaporation film rates from left and right films

$$
\begin{aligned}
& \dot{m}_{f, e, i}^{r, k}=\frac{U_{f} \wp}{\mathcal{L}} \int_{X_{f, i}^{r, k}}^{X_{e, i}^{r, k}}\left[T_{w}(x)-T_{\text {sat }, i}\right] \mathrm{d} x, \\
& \dot{m}_{f, e, i}^{l, k}=\frac{U_{f} \wp}{\mathcal{L}} \int_{X_{e, i}^{l, k}}^{X_{f, i}^{l, k}}\left[T_{w}(x)-T_{\text {sat }, i}\right] \mathrm{d} x .
\end{aligned}
$$

used for the definition of the film edge dynamics below. These equations are the multi-branch counterparts of Eq. (70). The vapor mass change caused by the condensation in the $k$-th effective condenser is

$$
\dot{m}_{f, c, i}^{k}=\frac{U_{f} \wp}{\mathcal{L}} \begin{cases}\int_{X_{i}^{l}}^{X_{e, i}^{l, k}}\left[T_{w}(x)-T_{s a t, i}\right] \mathrm{d} x, & \text { if } k=1 \\ \int_{X_{e, i}^{r, k-1}}^{X_{e, i}^{l, k}}\left[T_{w}(x)-T_{s a t, i}\right] \mathrm{d} x, & \text { if } 1<k \leq N_{e, i} \\ \int_{X_{e, i}^{r, k-1}}^{X_{i}^{r}}\left[T_{w}(x)-T_{s a t, i}\right] \mathrm{d} x, & \text { if } k=N_{e, i}+1\end{cases}
$$

The vapor mass change caused by the $s$-th $(s=l, r)$ meniscus phase change is (cf. Eq. 64)

$$
\dot{m}_{m, i}^{s}=\frac{U_{m} \wp L_{m}}{\mathcal{L}}\left[T_{w}\left(X_{i}^{s}\right)-T_{s a t, i}\right]
$$

The total phase change rate for the $i$-th bubble (cf. Eq. 63)

$$
\dot{m}_{i}=\dot{m}_{m, i}^{l}+\dot{m}_{m, i}^{r}+\dot{m}_{f, c, i}^{N_{e, i}+1}+\sum_{k=1}^{N_{e, i}}\left(\dot{m}_{f, e, i}^{l, k}+\dot{m}_{f, e, i}^{r, k}+\dot{m}_{f, c, i}^{k}\right)
$$

is a sum of masses taken from films (and also menisci) per second.

The film dynamics is described by the multi-branch coun- 
terparts of Eq. (43),

$$
\begin{aligned}
& \dot{X}_{f, i}^{r, k}= \begin{cases}\dot{X}_{i}^{r}, & \text { if } X_{f, i}^{r, k}=X_{i}^{r} \text { and } \dot{X}_{i}^{r} \leq 0 \\
\dot{X}_{i}^{l}, & \text { if } X_{f, i}^{r, k}=X_{i}^{l} \text { and } \dot{X}_{i}^{l} \geq 0 \\
\frac{\dot{m}_{f, c, i}^{k+1}}{2 \rho_{l} S_{f}}, & \text { if } X_{e, i}^{r, k}=X_{f, i}^{r, k} \text { and } X_{f, i}^{l, k}<X_{f, i}^{r, k} \\
\frac{\dot{m}_{f, e, i}^{r, k}}{\rho_{l} S_{f}}, & \text { otherwise }\end{cases} \\
& \dot{X}_{f, i}^{l, k}= \begin{cases}\dot{X}_{i}^{l}, & \text { if } X_{f, i}^{l, k}=X_{i}^{l} \text { and } \dot{X}_{i}^{l} \geq 0 \\
\dot{X}_{i}^{r}, & \text { if } X_{f, i}^{l, k}=X_{i}^{r} \text { and } \dot{X}_{i}^{r} \leq 0 \\
-\frac{\dot{m}_{f, c, i}^{k}}{2 \rho_{l} S_{f}}, & \text { if } X_{e, i}^{l, k}=X_{f, i}^{l, k} \text { and } X_{f, i}^{l, k}<X_{f, i}^{r, k} \\
-\frac{\dot{m}_{f, e, i}^{l, k}}{\rho_{l} S_{f}} . & \text { otherwise }\end{cases}
\end{aligned}
$$

The order of lines is meaningful (2nd option holds if the 1st is invalid, the $3 \mathrm{rd}$ if first two are invalid, etc.). The 1 st line of each of these formulas means the film absence (which implies $\dot{m}_{f, c, i}^{k}=\dot{m}_{f, e, i}^{s, k}=0$ ) and the plug advancing motion. The 2 nd line means the dry spot absence and the absence of the opposite film; the film junction situates at the opposite meniscus and moves with it. The 3rd line corresponds to the film advancing motion because of condensation when the whole film is in the effective condenser. Only a half of the condensed mass contributes to the left film elongation; the other half is used for the elongation of the right film of the next dry spot $(k+1-$ th). The 4 th line corresponds to the film edge retraction when the film edge is within the effective evaporator. The numerical software that implements this algorithm is called CASCO (Code Avancé de Simulation de Caloduc Oscillant: Advanced PHP simulation code in French) [127].

Daimaru et al. [33] have implemented the FEC model with the fluid solid interaction and bubble generation but limited to a single dry area per bubble. For the first time, they introduced into simulation the check valves and a simulation of the heat spreader that provided a thermal interaction between the different PHP branches. They studied the start-up that occurred without the temperature overshoot and obtained a steady regime. Due to the check valves, they obtained a true circulation regime of PHP. The agreement with experimental data was fairly good. However they could not make a detailed comparison because the experimental data lacked: their experiment was performed on board of a satellite. They also raised a very important question about the influence of the initial liquid plug distribution on the PHP start-up. They concluded that at low heating power the PHP would not start-up if the evaporator is initially dry. However, the start-up occurred under such a condition at a higher power.

The conservation of energy and mass in the system and the grid independence is important to be satisfied throughout the simulation and has been carefully verified in CASCO [73]. In a steady regime, the average heat power transferred from the evaporator should be equal to the amount received by the liquid: on average, there is no heat accumulation both in the tube wall and in the fluid. The sum $\dot{Q}_{e}$ of all the contributions (la- tent and sensible heat transfer through the fluid and the solid) should be equal to the input power $P_{e}$ within a small calculation error. Similarly, the total fluid mass that consists of the mass of plugs, films, and vapor should be conserved throughout the simulation. However, a small deviation always exists. The most evident reason is a tiny overlap of the film edges during the dry spot disappearance in the condenser and also of the menisci overlap during the bubble disappearance. Such an overlap appears because of the numerical error associated with the finiteness of the time step. The bubble generation-disappearance events are frequent, their number can be as large as $10^{6}$ during a run so the error grows with time. The numerical algorithms used in CASCO have been carefully worked out to minimize the mass deviation. Such consistency checks are necessary to simulate the steady regimes for massive PHP structures, which have long transient times.

CASCO is now stable and capable to simulate the PHP of various structures, in particular the multisource/multisink PHP with thermal spreaders. An example of such a device [140] is shown in Fig. 27. The structure of the 14-turn tubular aluminium PHP is specific [141], with three adiabatic sections per PHP turn between the evaporator and the first condenser, between two condensers, and between the second condenser and the evaporator section of the next turn (Fig. 27a). It is filled with FC-72 with $F R=0.5$. Since the thermal gradients are measured to be extremely low in the spreader, it is assumed isothermal. The PHP massive heater block $15 \times 12 \times 110 \mathrm{~mm}^{3}$ comprised an Al spreader sandwiched between two alumina heaters. Similarly to [33], it is simulated as an equivalent thermal mass $m_{e} c_{e}$ that was connected to the tubes through a thermal conductivity $U_{e}$ :

$$
m_{e} c_{e} \frac{\mathrm{d} T_{e}}{\mathrm{~d} t}=P_{e}-\wp_{o} \int q_{e}(x) \mathrm{d} x, \quad q_{e}(x)=U_{e}\left[T_{e}-T_{w}(x)\right] .
$$

The integration is performed over all the evaporator sections. The heat flux from the spreader is coupled to the PHP tube heat transfer problem through Eq. (15).

Because of the large diameter tubes $\left(d=3 \mathrm{~mm}>d_{c r}\right)$, the device cannot oscillate in the Earth gravity. To achieve the microgravity conditions, the prototype was tested during a parabolic flight. Unfortunately, the $20 \mathrm{~s}$ microgravity duration is too small to achieve a stable functioning regime so the transient simulation is required. The data are then dependent on the initial phase distribution, only some features of which are known since only one branch is transparent. One can only reasonably guess that (i) the liquid gathers at the evaporator side due to the vertical favorable orientation of PHP during the hyper-gravity period preceding microgravity and (ii) the internal tube walls are covered by a continuous liquid film formed during preceding parabolas. The thermal model of the PHP solid structure was adjusted by comparing with the of the empty PHP experimental data. The simulation data on the local tube temperature at the TC exact positions (Fig. 28a), plug velocities (Fig. 28b), plug temperature distribution, plug lengths etc. were compared to the experimental data with a good agreement.

The huge advantage of the modeling approaches is the accessibility of some parameters that are difficult to measure or 
vary experimentally. Because of this advantage, the simulation studies open a new perspective concerning the general issues of PHP functioning. For example, a key question of the PHP implementation concerns the PHP functioning regimes. Karthikeyan et al. [142] described the regimes of stable operation which are observed experimentally in the PHP: continuous functioning (oscillation or circulation), intermittent or stopover regime and dryout. One needs to place the PHP in the regime of continuous functioning to obtain the best performance and thus understand the parameters that control each of the regimes. Such studies should be started by identification of a scalar physical parameter (similar to the order parameter in the theory of phase transitions) that quantifies a difference between the low performance intermittent regime and high performance continuous functioning regime. By using CASCO simulations, such a parameter has been proposed [73]. It is the averaged in time fraction $\left\langle F_{l}\right\rangle$ of the wall belonging to the evaporator covered by the liquid plugs (not films). Indeed, it is drastically different in these regimes. For an example of the $30 \mathrm{~cm}$ long 10-turn copper-water PHP functioning at $P_{e}=200 \mathrm{~W}$ and $T_{c}=22^{\circ} \mathrm{C}$, $\left\langle F_{l}\right\rangle=0.04$ for the horizontal orientation where the intermittent regime is observed, and $\left\langle F_{l}\right\rangle=0.37$ for the vertical bottom heated orientation where the continuous oscillations occur.

\subsection{Models with varying film thickness}

An approach based on the variable thickness wedge film model (cf. sec. 2.3.6) was developed by d'Entremont and Thome $[59,60]$. The 4th order Runge-Kutta method was used for the time stepping. This model is for $L_{a}=0$ and the imposed constant temperatures of both evaporator and condenser. More recently, this model was updated to include the fluid-solid thermal coupling [143]. An extensive regime analysis has been performed for the R245fa fluid for different fill ratios and number $N_{p}$ of turns ranging from 1 (single loop PHP) to 24. The main functioning regime was small amplitude chaotic oscillations of large liquid plugs, one plug per turn in condenser section. The bubble generation was introduced. Instead of choosing fixed nucleation sites, a bubble was nucleated at a location of maximum liquid superheating that was searched inside each plug. Two its portions of the length $L_{n u c l, m i n}=2 \mathrm{~mm}$ adjacent to the menisci were excluded from the search. Once a large plug penetrated into the evaporator and the criterion (47) was satisfied, a bubble was nucleated. Since the evaporator wall temperature was constant, the bubble was nucleated always at the distance $L_{\text {nucl,min }}$ from the leading plug meniscus (see a discussion in sec. 2.4) since the superheat attained its maximum there. This means that a small liquid plug of the fixed length $L_{\text {nucl,min }}$ was cut by the nucleated bubble from the large plug. This small plug was propelled by the expanding bubble into the evaporator. While moving, the small plug left behind a liquid film thus shrinking in length. Typically, the small plug disappeared before crossing the whole evaporator. But sometimes it reached the opposite large plug and coalesce with it thus increasing temporarily the oscillation amplitude. According to the results of d'Entremont and Thome, the bubble generation introduced a perturbation to the motion of the large plugs that oscillated with a small amplitude, each in its own condenser section.
The two-turn PHP showed oscillations in the favorable vertical orientation (evaporator at the bottom). Probably under the action of gravity, the average position of liquid plugs in the condenser sections was shifted to one of the sides (both plugs to the same side). For this reason, they entered the condenser more often from one side than from another, which caused the birth and dissymmetric propulsion of small plugs, preferentially in one direction, so the average plug velocity was nonzero. They called such a regime circulation (the major part of the liquid did not circulate though). Such a dissymmetry was nonexistent at a larger turn number $N_{p}$, causing a reduction of the heat transfer rate per turn. The critical $N_{p}$ for (nearly) gravity-independent functioning situated between 5 and 15 depending on the regime.

However the vapor thermodynamic model of d'Entremont and Thome [59, 60] assumes that the vapor is always at saturation which is generally incorrect, see sec. 2.2.1 for the extended discussion.

The varying film thickness model of Senjaya and Inoue [106] is more complicated compared both to the FEC model and to the wedge film model since it introduces the flow equations in all three domains (liquid plug, vapor film and liquid film). Accordingly, the space and time dependent velocity and pressure in all three domains need to be found at each time step. With some minor modifications, it was recently implemented by Bae et al. [108] and Noh and Kim [131]. They compared the simulations based on this model to the FEC model (apparently, implemented with a unique dry spot per bubble) and found that both models successfully reproduce the overall experimentally found behavior both qualitatively (compare Figs. 25b and c) and quantitatively [108]. It is not thus clear why this much more complicated variable thickness approach is required instead of FEC model. Noh and Kim [131] have made the grid independence tests and a comparison of the varying film thickness model with the experimental results on the overall performance available in the literature and achieved an excellent agreement. They also performed simulations of several PHP configurations by constraining the external PHP sizes. They proposed a merit number $d^{2} N_{p}$ valid under such a constraint. It is yet to be applied to experimental results for validation.

\section{2D and 3D PHP simulations}

The direct numerical simulations (DNS) of PHP are much more intensive in terms of programming effort and the computer resources, and for this reason they begin to appear only recently. Within DNS, the full Navier-Stokes equations are solved. Comparing to 1D simulations, much more detailed information on PHP functioning can be obtained. This information can be useful not only by itself but can serve for improvement of 1D models. This concerns first of all the liquid-vapor interface morphology. As mentioned before, the main PHP flow regime is the plug-slug flow. However other flow regimes (like dispersed or annular flow) are sometimes encountered. DNS can describe all of them. Another important issue concerns the pressure drop during oscillations, and in particular involving the plugs in the channel bends (discussed in sec. 2.1). Such simulations can reveal the impact of liquid inertia on the menisci 
curvature that has a decisive impact on the deposited film thickness (sec. 2.3.1). The liquid heat transfer affected by the plug flow is a question of interest too (sec. 2.1.3). The plug flow in the rectangular flat plate PHP channel is also a major issue that can be studied. In such simulations, an information on the bubble and plug interactions can be obtained. This concerns the bubble generation that is not limited to the tube-size bubbles (sec. 2.4). The growth and collapse of smaller bubbles can be simulated to reveal their impact on the oscillations. Unlike the 1D approaches, the fluid mixtures can also be simulated. One can also obtain a useful information about the impact of surface morphology and wetting properties.

The dynamics is described by the full Navier-Stokes equations both in the vapor and liquid phases,

$$
\begin{aligned}
& \frac{\partial \rho}{\partial t}+\nabla \cdot(\rho \vec{u})=J \delta^{i} \\
& \frac{\partial \rho \vec{u}}{\partial t}+\nabla \cdot(\rho \vec{u} \vec{u})=-\nabla p+\nabla \cdot\left[\mu\left(\nabla \vec{u}+\nabla^{T} \vec{u}\right)\right]+\rho \vec{g}+\sigma K \vec{n} \delta^{i}
\end{aligned}
$$

$$
\rho \frac{\partial c_{p} T}{\partial t}+\rho \vec{u} \cdot \nabla\left(c_{p} T\right)=\frac{\partial p}{\partial t}+\vec{u} \cdot \nabla p+\nabla \cdot(\lambda \nabla T)-J \mathcal{L} \delta^{i},
$$

where $\delta^{i}$ is the Dirac delta function centered at the interface. It reflects the fact that the mass flux $J$ is injected into the vapor phase at evaporation. Note the ambiguity in many articles in which $\delta^{i}$ is omitted in writing of this equation so one has an impression that the evaporation occurs in the volume (which is actually an interfacial effect). The liquid is considered incompressible while the vapor obeys the ideal gas EOS so a compressible solver is necessary.

In addition to solving the Navier-Stokes equations in the volume, such simulations face a considerable difficulty: the free liquid-vapor interface numerical modeling. This requires a quite strong (both programming and computational) effort. There are several methods to describe the interface [144]: the volume of fluid method (VOF), the level-set method (LS), the phase-field method, the front-tracking method, the boundary element method, and their combinations. Generally, to be accurate, VOF requires a finer mesh while LS requires a smaller time step. The front-tracking method is generally the most accurate but it was not yet used for the PHP simulation. In addition, a fine meshing is generally necessary to describe thin liquid films. All these issues mean a necessity of commercial or freeware existing CFD codes for the PHP simulation community.

Givler and Martinez [118, 145] used the commercial CFD code Flow-3D to model the PHPs of increasing complexity in 2D and later in 3D. An extensive simulation work has been carried out. The simulations are described in detail and the values of relevant parameters are discussed. The constant heat flux at the evaporator and constant $T_{c}$ were imposed. The tube conduction was implemented. The liquid films are not modeled. The management of vapor is done according to a built-in model. Each bubble is described by a single temperature like in the 1D models discussed above. However, the saturation temperature at the meniscus is not imposed as a boundary condition. The vapor energy equation (Eq. (7) of Givler and Martinez [145]) is incorrect similarly to those discussed in sec. 2.2.2.

The work of Lin et al. [146] is probably the first where the ANSYS Fluent software was used for a 2D simulation of the 4turn PHP in the bottom heated mode. Two Fluent flow models were tried: the homogeneous mixture model and VOF. The grid independence analysis is presented. As for the performance, the mixture model gave a better agreement with the experiment. It is not however clear if the oscillations occurred in the homogeneous approach (it was likely to be the convection). However this model gave a better agreement with the experiment (within a few tens of percent) than VOF (where the error was of the order of $100 \%$ ). In the VOF approach, the bubble oscillations were not clearly observed; the functioning through the bubble nucleation and growth was described.

A single-turn (closed loop) PHP was analyzed by using Fluent in 2D by Wang et al. [147]. The fluid dynamics is solved in both phases and the films are resolved. The bubble generation is implemented. The choice of nucleation barrier (which is the most important parameter for the bubble generation, see sec. 2.4) is not discussed. The PHP dynamics is chaotic and the flow pattern looks realistic.

Ghanta and Pattamatta [148] used the OpenFoam freeware to perform the 2D simulations of the single branch PHP. The VOF solver of OpenFoam was augmented with the explicit interface reconstruction algorithm. This work is the only one where the numerical algorithm was validated at least for an isothermal case. The oscillations were not obtained (the simulation was stopped when the meniscus entered the condenser).

Pouryoussefi and Zhang [149, 150, 151] performed an extensive study of 2,4, and 5-turn PHP configurations in 2D and $3 \mathrm{D}$ by VOF. Dry area appearance is described. The flow was investigated by considering the Liapunov exponents and autocorrelation function of the flow fluctuations. It was concluded that the dynamic chaos occurs in PHP. Fluent was probably used (this is not specified). The employed computer resources were probably considerable.

Sedighi et al. [152] simulated a 4-branch PHP with an extra dead-end tube attached to the evaporator (to provide the circulation regime) in the bottom-heated mode. The $2 \mathrm{D}$ VOF OpenFoam approach was used with $\sim 10$ cells across the channel. Consequently, the films were not resolved. The simulation equations are not complete and the phase change model is not specified.

Xie et al. [153] used the VOF algorithm of Fluent to model the single-loop PHP. They studied the impact of the heating distribution and turn radius on the flow and oscillation frequency. The liquid films were not resolved either.

Wang et al. [154] applied the 3D VOF approach of Fluent to the closed-loop PHP simulation and studied the effect of the wall wetting properties (contact angle). They have implemented the bubble generation but the liquid films were not resolved either. They concluded that although the start-up is worse for the hydrophobic wall, the PHP performance in the steady regime is better than for hydrophilic wall for low fluxes. Conversely, the hydrophilic wall seems to be more advantageous for high heat fluxes. 
Barba et al. [155] used the VOF algorithm of Fluent in the $2 \mathrm{D}$ axisymmetric geometry to simulate the single-branch PHP. They resolved the film thickness and modeled its evaporation and condensation. The self-sustained oscillations could not however been obtained.

One needs to make several important remarks concerning the DNS of two-phase systems. This is an active research field by itself. The isothermal free interface simulation is well established. However the phase change simulation still presents a high degree of uncertainty and a separate validation of each model involved into the calculation is needed. However, typically, it is not done for the PHP simulations. This concerns in particular the interfacial phase change models that often involve artificial parameters. One can see this difficulty on an example of the Lee phase change model [156] implemented in Fluent and commonly used in OpenFoam. It contains the relaxation coefficient (that is mostly analogous to the inverse interfacial kinetic resistance (sec. 2.3.5). Its numerical value used for the PHP simulations is nonphysical. It is $\sim 0.3 \mathrm{~m}^{-1}$ [155], $0.1 \mathrm{~m}^{-1}$ [147, 153, 154], while the value that passes the test case of the Stefan problem (for which analytical solution is known) is rather $10^{6} \mathrm{~m}^{-1}$ [157]. In many PHP simulation works $[146,158,159]$ its value is used as a fitting parameter or is not specified at all.

Another point concerns the VOF method most commonly used in such simulations in spite of its main deficiency: artificial spurious currents that form near the interfaces, implying a necessity to control them with artificial algorithms. The front tracking method deprived (or almost deprived) of this deficiency should be used in conjunction with more physical phase change model based on the interfacial energy balance similar to Eq. (68).

In VOF, the interface is described in terms of the liquid volume fraction in a computation cell that varies continuously, so interface is at least several grid cells wide. In non-stationary problems, the interfacial mass flux obtained with the Lee model needs usually to be smeared over the interface thickness by using an artificial relaxation time to provide the numeric stability. For these reasons, the phase change validation should concern not only in the bulk part of the interface but also the film and CL regions which need to be specially treated (the fluid thickness from one side being insufficient).

The liquid film around the bubbles presents an additional challenge because of the strong shear in it, so the grid should be strongly refined near the wall to provide its adequate shape reconstruction. Another issue concerns the fluid-solid coupling that is absent in most simulations but is extremely important for the correct understanding of the PHP functioning (sec. 3.2.2).

\section{Conclusions}

Because of non-stationary functioning, the heat transfer correlations appear to have a poor prediction capability for the PHP case. The PHP dynamic simulation seems to be the only way of its reliable modeling. Predictive simulation tools capable to provide the optimization and design of industrial prototypes are required. While the direct numerical simulation in $2 \mathrm{D}$ or $3 \mathrm{D}$ can accurately describe different flow regimes inside the PHP, 1D simulations appear as a viable and less expensive alternative in terms of computer resources. Indeed, an industrially relevant PHP topology [159] requires at present the 2D simulation time as large as 4-6 weeks per run (as compared to one hour of the 1D CASCO code).

The 2D and 3D PHP models still lack reliability. The numerical algorithms used in such approaches should be separately validated on the test cases available in the literature (like Stefan and Scriven problems). The 2D and 3D models probably need to be seen as a valuable mean to obtain information for the development of 1D models rather than a PHP design tool. In particular, the information on the following phenomena related to the accelerated plug flow can be useful:

- Impact of liquid inertia on the menisci curvature that affects the deposited film thickness.

- Pressure drop and liquid heat transfer.

- Flow in the rectangular capillaries

Last decade, an understanding of the PHP functioning has begun to emerge. Contrary to what was believed initially, the heat transfer via the evaporation of thin liquid films has been recognized to be more important than the sensible heat exchange through the liquid plugs. It was predicted by simulation and then shown experimentally. This shows the importance of an adequate film modeling. One can distinguish several 1D simulation approaches that differ mainly by the description of the dynamic behavior of the vapor phase and the liquid films. Each of these approaches has shortcomings that yet need to be improved. The hydrodynamics of liquid films appears to be well understood. However the PHP film modeling appears to be a crucial bottleneck. New film models need to be developed in the future as a tradeoff between the simplicity and the physical description presented in this review.

Each PHP numerical model should be validated against existing experiments for the simplest case of one bubble (single branch PHP). Experiments with U-turn (two branch) PHP are strongly needed. Future PHP simulations should be validated not only with the overall evaporator temperature behavior observed experimentally but with more detailed data on the plug dynamics that can be obtained with the infra-red or optical visualization. It is relatively easy to obtain the stable multi-branch PHP oscillations by simulating the bottom heated mode. The PHP simulations should be tested for more critical regimes like horizontal orientation or top heating mode.

By modeling the single branch PHP, some analytical expressions can be obtained. In particular they show that the start-up threshold is defined by the phase change efficiency inversely proportional to the slope of saturation curve, and the energy dissipation in the system. In addition to the liquid viscosity contribution, the dissipation is provided by the vapor heat transfer. These findings are general and thus useful for the multi-branch PHP.

We are still do not understand the crucial PHP features like start-up threshold power and dryout limit as functions of the 
PHP structure. A deep study of the PHP functioning regimes is necessary to master these phenomena.

\section{Acknowledgements}

The present work was carried out in the framework of project TOPDESS, financed through the Microgravity Application Program by the European Space Agency. The financial support from CNES granted in the framework of the GDR MFA is acknowledged. The author thanks Dr. M. Rao and Dr. S. Lips for sharing their experimental data.

\section{References}

[1] H. Akachi, Structure of micro-heat pipe, US Patent 5219020, 1993. URL: https://www.lens.org/lens/patent/US_4921041_A.

[2] S. Khandekar, P. Charoensawan, M. Groll, P. Terdtoon, Closed loop pulsating heat pipes Part B: visualization and semi-empirical modeling, Appl. Therm. Eng. 23 (2003) $2021-2033$.

[3] S. Rittidech, P. Terdtoon, M. Murakami, P. Kamonpet, W. Jompakdee, Correlation to predict heat transfer characteristics of a closed-end oscillating heat pipe at normal operating condition, Appl. Therm. Eng. 23 (2003) $497-510$.

[4] T. Katpradit, T. Wongratanaphisan, P. Terdtoon, P. Kamonpet, A. Polchai, A. Akbarzadeh, Correlation to predict heat transfer characteristics of a closed end oscillating heat pipe at critical state, Appl. Therm. Eng. 25 (2005) $2138-2151$.

[5] G. Arslan, M. Özdemir, Correlation to predict heat transfer of an oscillating loop heat pipe consisting of three interconnected columns, Energy Convers. Manage. 49 (2008) 2337 - 2344.

[6] M. B. Shafii, S. Arabnejad, Y. Saboohi, H. Jamshidi, Experimental investigation of pulsating heat pipes and a proposed correlation, Heat Transfer Eng. 31 (2010) $854-861$.

[7] J. Qu, Q. Wang, Experimental study on the thermal performance of vertical closed-loop oscillating heat pipes and correlation modeling, Appl. Energy 112 (2013) $1154-1160$.

[8] M. Ebrahimi Dehshali, M. A. Nazari, M. B. Shafii, Thermal performance of rotating closed-loop pulsating heat pipes: Experimental investigation and semi-empirical correlation, Int. J. Therm. Sci. 123 (2018) $14-26$.

[9] S. Khandekar, X. Cui, M. Groll, Thermal performance modeling of pulsating heat pipes by artificial neural network, in: Proc. 12th Int. Heat Pipe Conf., Moscow, 2002, pp. 215 - 219.

[10] A. Jokar, A. A. Godarzi, M. Saber, M. B. Shafii, Simulation and optimization of a pulsating heat pipe using artificial neural network and genetic algorithm, Heat Mass Transf. 52 (2016) 2437 - 2445.

[11] M. Jalilian, H. Kargarsharifabad, A. Abbasi Godarzi, A. Ghofrani, M. B. Shafii, Simulation and optimization of pulsating heat pipe flat-plate solar collectors using neural networks and genetic algorithm: a semiexperimental investigation, Clean Technol. Environ. Policy 18 (2016) $2251-2264$.

[12] X. Wang, Y. Yan, X. Meng, G. Chen, A general method to predict the performance of closed pulsating heat pipe by artificial neural network, Appl. Therm. Eng. 157 (2019) 113761.

[13] Y. Zhang, A. Faghri, Advances and unsolved issues in pulsating heat pipes, Heat Transfer Eng. 29 (2008) 20 - 44.

[14] S. Khandekar, P. K. Panigrahi, F. Lefèvre, J. Bonjour, Local hydrodynamics of flow in a pulsating heat pipe: a review, Frontiers in Heat Pipes 1 (2010) 023003.

[15] D. Bastakoti, H. Zhang, D. Li, W. Cai, F. Li, An overview on the developing trend of pulsating heat pipe and its performance, Appl. Therm. Eng. 141 (2018) $305-332$.

[16] M. A. Nazari, M. H. Ahmadi, R. Ghasempour, M. B. Shafii, How to improve the thermal performance of pulsating heat pipes: A review on working fluid, Renewable Sustainable Energy Rev. 91 (2018) 630 - 638.
[17] M. Marengo, V. Nikolayev, Pulsating heat pipes: Experimental analysis, design and applications, in: J. R. Thome (Ed.), Encyclopedia of TwoPhase Heat Transfer and Flow IV, volume 1: Modeling of Two-Phase Flows and Heat Transfer, World Scientific, 2018, pp. $1-62$.

[18] V. Nikolayev, M. Marengo, Pulsating heat pipes: Basics of functioning and numerical modeling, in: J. R. Thome (Ed.), Encyclopedia of TwoPhase Heat Transfer and Flow IV, volume 1: Modeling of Two-Phase Flows and Heat Transfer, World Scientific, 2018, pp. 63 - 139.

[19] H. Ma, Oscillating Heat Pipes, Springer, New York, 2015.

[20] G. Spinato, N. Borhani, J. R. Thome, Operational regimes in a closed loop pulsating heat pipe, Int. J. Therm. Sci. 102 (2016) $78-88$.

[21] L. Pietrasanta, M. Mameli, D. Mangini, A. Georgoulas, N. Michè, S. Filippeschi, M. Marengo, Developing flow pattern maps for accelerated two-phase capillary flows, Exp. Therm Fluid Sci. 112 (2020) 109981.

[22] G. Lagubeau, Propulsion par moteur pop-pop, Master's thesis, supervisor: D. Quéré, PMMH-ESPCI, Paris, 2006.

[23] F. P. Bretherton, The motion of long bubbles in tubes, J. Fluid Mech. 10 (1961) $166-188$.

[24] R. Gupta, D. Fletcher, B. Haynes, Taylor flow in microchannels: A review of experimental and computational work, J. Comput. Multiphase Flows 2 (2010) $1-31$.

[25] V. Talimi, Y. S. Muzychka, S. Kocabiyik, A review on numerical studies of slug flow hydrodynamics and heat transfer in microtubes and microchannels, Int. J. Multiphase Flow 39 (2012) 88 - 104.

[26] C. Baldassari, M. Marengo, Flow boiling in microchannels and microgravity, Prog. Energy Combust. Sci. 39 (2013) 1 - 36.

[27] A. Cherukumudi, E. Klaseboer, S. A. Khan, R. Manica, Prediction of the shape and pressure drop of Taylor bubbles in circular tubes, Microfluid. Nanofluid. 19 (2015) 1221 - 1233.

[28] M. T. Kreutzer, F. Kapteijn, J. A. Moulijn, C. R. Kleijn, J. J. Heiszwolf, Inertial and interfacial effects on pressure drop of Taylor flow in capillaries, AlChE J. 51 (2005) 2428 - 2440.

[29] T. S. Zhao, P. Cheng, Experimental studies on the onset of turbulence and frictional losses in an oscillatory turbulent pipe flow, Int. J. Heat Fluid Flow 17 (1996) 356 - 362.

[30] H. Schlichting, Boundary-Layer Theory, McGraw-Hill, Ney York, 1968.

[31] M. B. Shafii, A. Faghri, Y. Zhang, Thermal modeling of unlooped and looped pulsating heat pipes, J. Heat Transfer 123 (2001) 1159 - 1172.

[32] V. S. Nikolayev, A dynamic film model of the pulsating heat pipe, J. Heat Transfer 133 (2011) 081504.

[33] T. Daimaru, H. Nagai, M. Ando, K. Tanaka, A. Okamoto, H. Sugita, Comparison between numerical simulation and on-orbit experiment of oscillating heat pipes, Int. J. Heat Mass Transfer 109 (2017) 791 - 806.

[34] M. Mameli, M. Marengo, S. Zinna, Numerical model of a multi-turn closed loop pulsating heat pipe: Effects of the local pressure losses due to meanderings, Int. J. Heat Mass Transfer 55 (2012) 1036- 1047.

[35] R. Darby, R. P. Chhabra, Chemical Engineering Fluid Mechanics, 3rd ed ed., CRC Press, 2017.

[36] I. Idelchik, Handbook of Hydraulic Resistance, Jaico Publishing House, 2008.

[37] U. H. Kurzweg, L. de Zhao, Heat transfer by high-frequency oscillations: A new hydrodynamic technique for achieving large effective thermal conductivities, Phys. Fluids 27 (1984) $2624-2627$.

[38] T. Zhao, P. Cheng, A numerical solution of laminar forced convection in a heated pipe subjected to a reciprocating flow, Int. J. Heat Mass Transfer 38 (1995) 3011 - 3022.

[39] P. Li, K. T. Yang, Mechanisms for the heat transfer enhancement in zeromean oscillatory flows in short channels, Int. J. Heat Mass Transfer 43 (2000) $3551-3566$

[40] H. N. Hemida, M. N. Sabry, A. Abdel-Rahim, H. Mansour, Theoretical analysis of heat transfer in laminar pulsating flow, Int. J. Heat Mass Transfer 45 (2002) $1767-1780$.

[41] J.-C. Yu, Z.-X. Li, T. S. Zhao, An analytical study of pulsating laminar heat convection in a circular tube with constant heat flux, Int. J. Heat Mass Transfer 47 (2004) 5297 - 5301.

[42] D. Yin, H. Ma, Analytical solution of oscillating flow in a capillary tube, Int. J. Heat Mass Transfer 66 (2013) 699 - 705.

[43] D. Yin, H. B. Ma, Analytical solution of heat transfer of oscillating flow at a triangular pressure waveform, Int. J. Heat Mass Transfer 70 (2014) $46-53$.

[44] B. Mehta, S. Khandekar, Local experimental heat transfer of single- 
phase pulsating laminar flow in a square mini-channel, Int. J. Therm. Sci. 91 (2015) 157 - 166.

[45] V. Gnielinski, New equations for heat and mass transfer in turbulent pipe and channel flow, Int. Chem. Eng. 16 (1976) 359 - 368.

[46] B. Holley, A. Faghri, Analysis of pulsating heat pipe with capillary wick and varying channel diameter, Int. J. Heat Mass Transfer 48 (2005) 2635 -2651 .

[47] Z. Che, T. N. Wong, N.-T. Nguyen, Heat transfer in plug flow in cylindrical microcapillaries with constant surface heat flux, Int. J. Therm. Sci. 64 (2013) $204-212$.

[48] M. Magnini, B. Pulvirenti, J. Thome, Numerical investigation of hydrodynamics and heat transfer of elongated bubbles during flow boiling in a microchannel, Int. J. Heat Mass Transfer 59 (2013) 451 - 471.

[49] B. Mehta, S. Khandekar, Measurement of local heat transfer coefficient during gas-liquid Taylor bubble train flow by infra-red thermography, Int. J. Heat Fluid Flow 45 (2014) 41 - 52

[50] V. Srinivasan, V. Marty-Jourjon, S. Khandekar, F. Lefèvre, J. Bonjour, Evaporation of an isolated liquid plug moving inside a capillary tube, Int. J. Heat Mass Transfer 89 (2015) 176 - 185.

[51] M. Morisaki, S. Minami, K. Miyazaki, T. Yabuki, Direct local heat flux measurement during water flow boiling in a rectangular minichannel using a MEMS heat flux sensor, Exp. Therm Fluid Sci. 121 (2021) 110285.

[52] C. Smoot, H. B. Ma, Numerical analysis of a vapor bubble oscillating in a capillary channel, J. Thermophys. Heat Transfer 31 (2017) $154-164$.

[53] V. P. Carey, Liquid-Vapor Phase Change Phenomena, Hemisphere, Washington D.C., 1992.

[54] P. Gully, F. Bonnet, V. S. Nikolayev, N. Luchier, T. Q. Tran, Evaluation of the vapor thermodynamic state in PHP, Heat Pipe Science and Technology 5 (2014) $369-376$.

[55] M. Rao, F. Lefèvre, S. Khandekar, J. Bonjour, Heat and mass transfer mechanisms of a self-sustained thermally driven oscillating liquidvapour meniscus, Int. J. Heat Mass Transfer 86 (2015) 519 - 530.

[56] H. Y. Noh, S. J. Kim, Thermal characterization and optimization of pulsating heat pipes operating in a circulation mode, Int. J. Heat Mass Transfer 115 (2017) 1234 - 1246.

[57] S. Jun, S. J. Kim, Experimental investigation on the thermodynamic state of vapor plugs in pulsating heat pipes, Int. J. Heat Mass Transfer 134 (2019) $321-328$.

[58] V. S. Nikolayev, I. Nekrashevych, Vapor thermodynamics and fluid merit for pulsating heat pipe, in: Proc. 19th Int. Heat Pipe Conf. and 13th Int. Heat Pipe Symp., Pisa, Italy, 2018.

[59] B. P. d'Entremont, J. R. Thome, A dynamic PHP model with transient evaporating films, in: Proc. 9th Int. Conf. Boiling and Condensation Heat Transfer, Boulder, USA, 2015.

[60] B. P. d'Entremont, J. R. Thome, A numerical study of pulsating heat pipe performance, in: Proc.InterPACKICNMM 2015, 2015, p. V003T10A025.

[61] V. S. Nikolayev, Comment on "Flow and heat transfer of liquid plug and neighboring vapor slugs in a pulsating heat pipe" by Yuan, Qu, \& Ma, Int. J. Heat Mass Transfer 54 (2011) 2226 - 2227.

[62] Y. Zhang, A. Faghri, M. B. Shafii, Analysis of liquid-vapor pulsating flow in a U-shaped miniature tube, Int. J. Heat Mass Transfer 45 (2002) $2501-2508$.

[63] R. T. Dobson, Theoretical and experimental modelling of an open oscillatory heat pipe including gravity, Int. J. Therm. Sci. 43 (2004) 113 119.

[64] G. Gürsel, A. J. H. Frijns, F. G. A. Homburg, A. A. van Steenhoven, A mass-spring-damper model of a pulsating heat pipe with a non-uniform and asymmetric filling, Appl. Therm. Eng. 91 (2015) 80 - 90.

[65] M. Manzoni, M. Mameli, C. de Falco, L. Araneo, S. Filippeschi, M. Marengo, Non equilibrium lumped parameter model for pulsating heat pipes: validation in normal and hyper-gravity conditions, Int. J. Heat Mass Transfer 97 (2016) 473 - 485.

[66] M. Manzoni, M. Mameli, C. de Falco, L. Araneo, S. Filippeschi, M. Marengo, Advanced numerical method for a thermally induced slug flow: application to a capillary closed loop pulsating heat pipe, Int. J. Numer. Methods Fluids 82 (2016) 375 - 397.

[67] R. Senjaya, T. Inoue, Oscillating heat pipe simulation considering bubble generation. Part I: Presentation of the model and effects of a bubble generation, Int. J. Heat Mass Transfer 60 (2013) 816 - 824.
[68] P. Sakulchangsatjatai, P. Chareonsawan, T. Waowaew, P. Terdtoon, M. Murakami, Mathematical modeling of closed-end pulsating heat pipes operating with a bottom heat mode, Heat Transfer Eng. 29 (2008) $239-254$.

[69] D. Yuan, W. Qu, T. Ma, Flow and heat transfer of liquid plug and neighboring vapor slugs in a pulsating heat pipe, Int. J. Heat Mass Transfer 53 (2010) $1260-1268$

[70] P. Cheng, H. Ma, A mathematical model of an oscillating heat pipe, Heat Transfer Eng. 32 (2011) 1037-1046.

[71] J. Jo, J. Kim, S. J. Kim, Experimental investigations of heat transfer mechanisms of a pulsating heat pipe, Energy Convers. Manage. 181 (2019) $331-341$.

[72] C. Kamijima, Y. Yoshimoto, Y. Abe, S. Takagi, I. Kinefuchi, Relating the thermal properties of a micro pulsating heat pipe to the internal flow characteristics via experiments, image recognition of flow patterns and heat transfer simulations, Int. J. Heat Mass Transfer 163 (2020) 120415.

[73] I. Nekrashevych, V. S. Nikolayev, Pulsating heat pipe simulations: impact of PHP orientation, Microgravity Sci. Technol. 31 (2019) 241 248. Issue on Heat pipe systems for thermal management in space.

[74] L. Fourgeaud, E. Ercolani, J. Duplat, P. Gully, V. S. Nikolayev, Evaporation-driven dewetting of a liquid film, Phys. Rev. Fluids 1 (2016) 041901.

[75] L. Fourgeaud, V. S. Nikolayev, E. Ercolani, J. Duplat, P. Gully, In situ investigation of liquid films in pulsating heat pipe, Appl. Therm. Eng. 126 (2017) 1023 - 1028.

[76] L. Fourgeaud, E. Ercolani, J. Duplat, P. Gully, V. S. Nikolayev, 3D reconstruction of dynamic liquid film shape by optical grid deflection method, Eur. Phys. J. E 41 (2018) 5.

[77] Y. Zhang, A. Faghri, Heat transfer in a pulsating heat pipe with open end, Int. J. Heat Mass Transfer 45 (2002) 755 - 764.

[78] W. Shao, Y. Zhang, Effects of film evaporation and condensation on oscillatory flow and heat transfer in an oscillating heat pipe, J. Heat Transfer 133 (2011) 042901.

[79] A. Oron, S. H. Davis, S. G. Bankoff, Long-scale evolution of thin liquid films, Rev. Mod. Phys. 69 (1997) 931-980.

[80] V. S. Nikolayev, Dynamics of the triple contact line on a nonisothermal heater at partial wetting, Phys. Fluids 22 (2010) 082105.

[81] L. D. Landau, B. V. Levich, Dragging of a liquid by a moving plate, Acta physico-chimica USSR 17 (1942) $42-54$.

[82] V. S. Nikolayev, S. Sundararaj, Oscillating menisci and liquid films at evaporation/condensation, Heat Pipe Sci. Technol. 5 (2014) 59 - 67.

[83] Y. Han, N. Shikazono, Measurement of the liquid film thickness in micro tube slug flow, Int. J. Heat Fluid Flow 30 (2009) 842 - 853.

[84] X. Zhang, V. S. Nikolayev, Liquid film dynamics with immobile contact line during meniscus oscillation, J. Fluid Mech. (2021). Submitted.

[85] J. Snoeijer, B. Andreotti, G. Delon, M. Fermigier, Relaxation of a dewetting contact line. Part 1. A full-scale hydrodynamic calculation, J. Fluid Mech. 579 (2007) $63-83$.

[86] P. Gao, A. Liu, J. J. Feng, H. Ding, X.-Y. Lu, Forced dewetting in a capillary tube, J. Fluid Mech. 859 (2019) $308-320$.

[87] T. S. Chan, J. H. Snoeijer, J. Eggers, Theory of the forced wetting transition, Phys. Fluids 24 (2012) 072104.

[88] J. H. Snoeijer, G. Delon, M. Fermigier, B. Andreotti, Avoided critical behavior in dynamically forced wetting, Phys. Rev. Lett. 96 (2006) 174504.

[89] P.-G. de Gennes, F. Brochard-Wyart, D. Quéré, Capillarity and Wetting Phenomena: Drops, Bubbles, Pearls, Waves, Springer, New York, 2004.

[90] S. Iliev, N. Pesheva, V. S. Nikolayev, Contact angle hysteresis and pinning at periodic defects in statics, Phys. Rev. E 90 (2014) 012406.

[91] V. S. Nikolayev, Dynamics and depinning of the triple contact line in the presence of periodic surface defects, J. Phys. Cond. Matt. 17 (2005) $2111-2119$.

[92] L. Fourgeaud, Analysis of the liquid film dynamics in a Pulsating Heat Pipe, Ph.D. thesis, Université Grenoble-Alpes, 2016. URL: https : // tel.archives-ouvertes.fr/tel-01409530.

[93] H. Wong, C. J. Radke, S. Morris, The motion of long bubbles in polygonal capillaries. Part 1. Thin films, J. Fluid Mech. 292 (1995) 71 - 94.

[94] A. Takawale, S. Abraham, A. Sielaff, P. S. Mahapatra, A. Pattamatta, P. Stephan, A comparative study of flow regimes and thermal performance between flat plate pulsating heat pipe and capillary tube pulsating heat pipe, Appl. Therm. Eng. 149 (2019) 613 - 624. 
[95] Y. J. Youn, Y. Han, N. Shikazono, Liquid film thicknesses of oscillating slug flows in a capillary tube, Int. J. Heat Mass Transfer 124 (2018) 543 -551 .

[96] S. Lips, A. Bensalem, Y. Bertin, V. Ayel, C. Romestant, J. Bonjour, Experimental evidences of distinct heat transfer regimes in pulsating heat pipes (PHP), Appl. Therm. Eng. 30 (2010) 900 - 907.

[97] S. Signé Mamba, J. C. Magniez, F. Zoueshtiagh, M. Baudoin, Dynamics of a liquid plug in a capillary tube under cyclic forcing: memory effects and airway reopening, J. Fluid Mech. 838 (2018) 165 - 191

[98] E. Lauga, M. P. Brenner, H. A. Stone, Microfluidics: The no-slip boundary condition, in: C. Tropea, A. Yarin, J. Foss (Eds.), Springer Handbook of Experimental Fluid Dynamics, Springer, New York, 2007, pp. 1217 - 1240. Https://arxiv.org/pdf/cond-mat/0501557.pdf.

[99] F. Brochard-Wyart, J.-M. Di Meglio, D. Quere, P.-G. de Gennes, Spreading of nonvolatile liquids in a continuum picture, Langmuir 7 (1991) 335 -338 .

[100] J. Eggers, Existence of receding and advancing contact lines, Phys. Fluids 17 (2005) 082106

[101] J. H. Snoeijer, J. Eggers, Asymptotic analysis of the dewetting rim, Phys. Rev. E 82 (2010) 056314.

[102] L. M. Pismen, J. Eggers, Solvability condition for the moving contact line, Phys. Rev. E 78 (2008) 056304.

[103] J. H. Snoeijer, B. Andreotti, Moving contact lines: Scales, regimes, and dynamical transitions, Annu. Rev. Fluid Mech. 45 (2013) 269 - 292.

[104] M. Rao, F. Lefèvre, P.-C. Czujko, S. Khandekar, J. Bonjour, Numerical and experimental investigations of thermally induced oscillating flow inside a capillary tube, Int. J. Therm. Sci. 115 (2017) 29 - 42.

[105] J. R. Thome, V. Dupont, A. M. Jacobi, Heat transfer model for evaporation in microchannels. Part I: presentation of the model, Int. J. Heat Mass Transfer 47 (2004) 3375 - 3385.

[106] R. Senjaya, T. Inoue, Oscillating heat pipe simulation considering dryout phenomena, Heat Mass Transfer 50 (2014) 1429 - 1441.

[107] N. Chauris, V. Ayel, Y. Bertin, C. Romestant, Evaporation of a liquid film deposited on a capillary heated tube: Experimental analysis by infrared thermography of its thermal footprint, Int. J. Heat Mass Transfer 86 (2015) $492-507$

[108] J. Bae, S. Y. Lee, S. J. Kim, Numerical investigation of effect of film dynamics on fluid motion and thermal performance in pulsating heat pipes, Energy Convers. Manage. 151 (2017) 296 - 310.

[109] M. Potash, P. C. Wayner, Evaporation from a two-dimensional extended meniscus, Int. J. Heat Mass Transfer 15 (1972) 1851 - 1863.

[110] P. Stephan, J. Hammer, A new model for nucleate boiling heat transfer, Heat Mass Transfer 30 (1994) 119 - 125.

[111] V. S. Nikolayev, D. A. Beysens, Boiling crisis and non-equilibrium drying transition, Europhys. Lett. 47 (1999) 345 - 351

[112] V. Janeček, V. S. Nikolayev, Apparent-contact-angle model at partial wetting and evaporation: impact of surface forces, Phys. Rev. E 87 (2013) 012404.

[113] V. Janeček, V. S. Nikolayev, Triggering the boiling crisis: a study of the dry spot spreading mechanism, Interfacial Phenom. Heat Transf. 2 (2014) $363-383$.

[114] R. Raj, C. Kunkelmann, P. Stephan, J. Plawsky, J. Kim, Contact line behavior for a highly wetting fluid under superheated conditions, Int. J. Heat Mass Transfer 55 (2012) 2664 - 2675

[115] V. Janeček, V. S. Nikolayev, Contact line singularity at partial wetting during evaporation driven by substrate heating, Europhys. Lett. 100 (2012) 14003

[116] V. Janeček, B. Andreotti, D. Pražák, T. Bárta, V. S. Nikolayev, Moving contact line of a volatile fluid, Phys. Rev. E 88 (2013) 060404

[117] D. Khrustalev, A. Faghri, Thick-film phenomenon in high-heat-flux evaporation from cylindrical pores, J. Heat Transfer 119 (1997) 272 $-278$.

[118] R. C. Givler, M. J. Martinez, Modeling of pulsating heat pipes, Technical Report SAND2009-4520, Sandia Nat. Lab., 2009.

[119] P. F. Pai, H. Peng, H. Ma, Thermomechanical finite-element analysis and dynamics characterization of three-plug oscillating heat pipes, Int J. Heat Mass Transfer 64 (2013) 623 - 635

[120] H. Peng, P. F. Pai, H. Ma, Nonlinear thermomechanical finite-element modeling, analysis and characterization of multi-turn oscillating heat pipes, Int. J. Heat Mass Transfer 69 (2014) $424-437$.

[121] D. Y. Han, X. Sun, Z. H. Gan, R. Y. Luo, J. M. Pfotenhauer, B. Jiao, Nu- merical investigation on pulsating heat pipes with nitrogen or hydrogen, IOP Conf. Ser.: Mater. Sci. Eng. 278 (2017) 012056.

[122] S. P. Das, V. S. Nikolayev, F. Lefèvre, B. Pottier, S. Khandekar, J. Bonjour, Thermally induced two-phase oscillating flow inside a capillary tube, Int. J. Heat Mass Transfer 53 (2010) 3905 - 3913.

[123] M. Dilawar, A. Pattamatta, A parametric study of oscillatory two-phase flows in a single turn pulsating heat pipe using a non-isothermal vapor model, Appl. Therm. Eng. 51 (2013) 1328 - 1338.

[124] T. Daimaru, S. Yoshida, H. Nagai, Study on thermal cycle in oscillating heat pipes by numerical analysis, Appl. Therm. Eng. 113 (2017) 1219 1227.

[125] A. Tessier-Poirier, T. Monin, E. Léveillé, S. Monfray, F. Formosa, L. G. Fréchette, How evaporation and condensation lead to self-oscillations in the single-branch pulsating heat pipe, Phys. Rev. Fluids 4 (2019) 103901.

[126] V. S. Nikolayev, Effect of tube heat conduction on the single branch pulsating heat pipe start-up, Int. J. Heat Mass Transfer 95 (2016) 477 487.

[127] I. Nekrashevych, V. S. Nikolayev, Effect of tube heat conduction on the pulsating heat pipe start-up, Appl. Therm. Eng. 117 (2017) 24 - 29

[128] T. Nagasaki, Y. Sawada, S. Hojo, Y. Ito, Study on the mechanism of liquid column oscillation in a pulsating heat pipe, in: Proc. Nat. Heat Transfer Symp., Okayama, Japan, 2011, p. 87. In Japaneese.

[129] R. Nemati, M. B. Shafii, Advanced heat transfer analysis of a U-shaped pulsating heat pipe considering evaporative liquid film trailing from its liquid slug, Appl. Therm. Eng. 138 (2018) 475 - 489.

[130] R. Senjaya, T. Inoue, Oscillating heat pipe simulation considering bubble generation. Part II: Effects of fitting and design parameters, Int. J. Heat Mass Transfer 60 (2013) 825 - 835 .

[131] H. Y. Noh, S. J. Kim, Numerical simulation of pulsating heat pipes: parametric investigation and thermal optimization, Energy Convers. Manage. 203 (2020) 112237.

[132] M. Andredaki, A. Georgoulas, N. Miche, M. Marengo, Numerical investigation of liquid film instabilities and evaporation in confined oscillating slug-plug flows, in: WIT Transactions on Engineering Sciences, volume 123, 2019, pp. $127-138$

[133] M. Rao, F. Lefèvre, S. Khandekar, J. Bonjour, Understanding transport mechanism of a self-sustained thermally driven oscillating two-phase system in a capillary tube, Int. J. Heat Mass Transfer 65 (2013) 451 459.

[134] V. Recklin, A. Pattamatta, P. Stephan, Experimental investigation on the thermo-hydrodynamics of oscillatory meniscus in a capillary tube using FC-72 as working fluid, Int. J. Multiphase Flow 75 (2015) $82-87$.

[135] V. S. Nikolayev, Oscillatory instability of the gas-liquid meniscus in a capillary under the imposed temperature difference, Int. J. Heat Mass Transfer 64 (2013) 313 - 321.

[136] B. S. Taft, A. D. Williams, B. L. Drolen, Review of pulsating heat pipe working fluid selection, J. Thermophys Heat Transfer 26 (2012) 651 656.

[137] J. Kim, S. J. Kim, Experimental investigation on working fluid selection in a micro pulsating heat pipe, Energy Convers. Manage. 205 (2020) 112462

[138] P. Sakulchangsatjatai, P. Terdtoon, T. Wongratanaphisan, P. Kamonpet, M. Murakami, Operation modeling of closed-end and closed-loop oscillating heat pipes at normal operating condition, Appl. Therm. Eng. 24 (2004) $995-1008$

[139] A. Yoon, S. J. Kim, Characteristics of oscillating flow in a micro pulsating heat pipe: Fundamental-mode oscillation, Int. J. Heat Mass Transfer 109 (2017) $242-253$.

[140] M. Abela, M. Mameli, V. Nikolayev, S. Filippeschi, Comparison of experiments and simulations on large diameter PHP in microgravity environment, in: Proc. Int. Symp. Oscillating/Pulsating Heat Pipes (ISOPHP), Daejeon, Korea, 2019.

[141] M. Mameli, A. Catarsi, D. Mangini, L. Pietrasanta, N. Michè, M. Marengo, P. Di Marco, S. Filippeschi, Start-up in microgravity and local thermodynamic states of a hybrid loop thermosyphon/pulsating heat pipe, Appl. Therm. Eng. 158 (2019) 113771.

[142] V. Karthikeyan, S. Khandekar, B. Pillai, P. K. Sharma, Infrared thermography of a pulsating heat pipe: Flow regimes and multiple steady states, Appl. Therm. Eng. 62 (2014) $470-480$.

[143] P. Aubin, B. D’Entremont, F. Cataldo, J. B. Marcinichen, R. L. Amalfi, 
J. R. Thome, Numerical simulations of pulsating heat pipes, part 1: Modeling, in: 2019 18th IEEE Intersociety Conf. on Thermal and Thermomechanical Phenomena in Electronic Systems (ITherm), Las Vegas, USA, 2019, pp. $232-242$.

[144] M. Wörner, Numerical modeling of multiphase flows in microfluidics and micro process engineering: a review of methods and applications, Microfluid. Nanofluid. 12 (2012) $841-886$.

[145] R. C. Givler, M. J. Martinez, Computational Model of Miniature Pulsating Heat Pipes, Technical Report SAND2012-4750, Sandia Nat. Lab., 2013.

[146] Z. Lin, S. Wang, R. Shirakashi, L. W. Zhang, Simulation of a miniature oscillating heat pipe in bottom heating mode using CFD with unsteady modeling, Int. J. Heat Mass Transfer 57 (2013) 642 - 656.

[147] J. Wang, H. Ma, Q. Zhu, Effects of the evaporator and condenser length on the performance of pulsating heat pipes, Appl. Therm. Eng. 91 (2015) $1018-1025$.

[148] N. Ghanta, A. Pattamatta, Modeling of compressible phase-change heat transfer in a taylor-bubble with application to pulsating heat pipe (PHP), Numer. Heat Transf. A 69 (2016) 1355 - 1375.

[149] S. M. Pouryoussefi, Y. Zhang, Numerical investigation of chaotic flow in a 2D closed-loop pulsating heat pipe, Appl. Therm. Eng. 98 (2016) $617-627$.

[150] S. M. Pouryoussefi, Y. Zhang, Nonlinear analysis of chaotic flow in a three-dimensional closed-loop pulsating heat pipe, J. Heat Transfer 138 (2016) 122003.

[151] S. M. Pouryoussefi, Y. Zhang, Analysis of chaotic flow in a 2D multiturn closed-loop pulsating heat pipe, Appl. Therm. Eng. 126 (2017) $1069-1076$.

[152] E. Sedighi, A. Amarloo, B. Shafii, Numerical and experimental investigation of flat-plate pulsating heat pipes with extra branches in the evaporator section, Int. J. Heat Mass Transfer 126 (2018) 431 - 441.

[153] F. Xie, X. Li, P. Qian, Z. Huang, M. Liu, Effects of geometry and multisource heat input on flow and heat transfer in single closed-loop pulsating heat pipe, Appl. Therm. Eng. 168 (2020) 114856.

[154] J. Wang, J. Xie, X. Liu, Investigation of wettability on performance of pulsating heat pipe, Int. J. Heat Mass Transfer 150 (2020) 119354.

[155] M. Barba, R. Bruce, B. Baudouy, Numerical simulation of the thermal and fluid-dynamic behavior of a cryogenic capillary tube, Cryogenics 106 (2020) 103044.

[156] W. H. Lee, A pressure iteration scheme for two-phase modeling, Technical Report LA-UR 79-975, Los Alamos Scientific Laboratory, Los Alamos, New Mexico, 1979.

[157] H. Liu, J. Tang, L. Sun, Z. Mo, G. Xie, An assessment and analysis of phase change models for the simulation of vapor bubble condensation, Int. J. Heat Mass Transfer 157 (2020) 119924.

[158] E. Jiaqiang, X. Zhao, Y. Deng, H. Zhu, Pressure distribution and flow characteristics of closed oscillating heat pipe during the starting process at different vacuum degrees, Appl. Therm. Eng. 93 (2016) 166 - 173.

[159] A. Mucci, F. K. Kholi, J. Chetwynd-Chatwin, M. Y. Ha, J. K. Min, Numerical investigation of flow instability and heat transfer characteristics inside pulsating heat pipes with different numbers of turns, Int. J. Heat Mass Transfer 169 (2021) 120934.

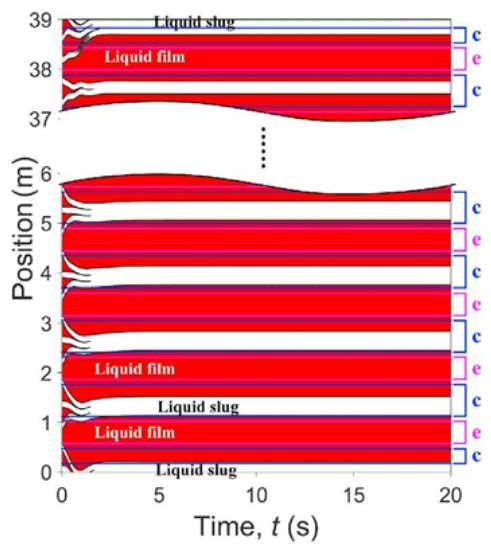

(a) Superheated vapor model result.

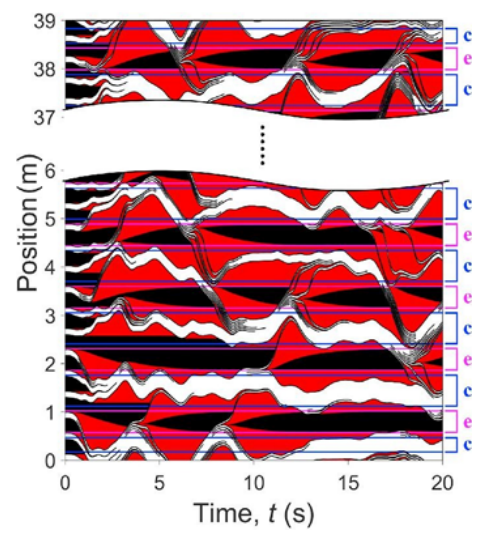

(b) FEC model.

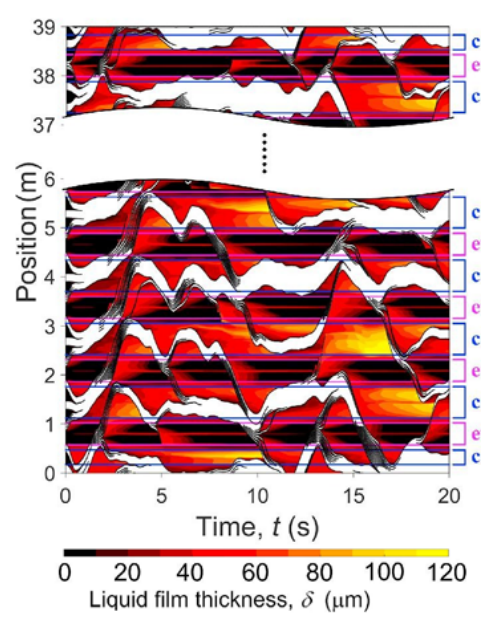

(c) Variable film thickness Senjaya and Inoue [106] model.

Figure 25: Dynamics of the $x$ positions of menisci and film edges for the 30turn horizontal PHP $\left(\mathrm{R}-134 \mathrm{a}, \mathrm{FR}=40 \%, T_{c}=10^{\circ} \mathrm{C}, T_{e}=14^{\circ} \mathrm{C}\right)$ simulated with different models (adapted from [108]). Only two portions of the channel (0-6 $\mathrm{m}$ and 37-39 $\mathrm{m})$ are pictured with an interruption between them. The liquid thickness is shown with color scale shown in Fig. 25c. The black color corresponds to the dry area, and the white portions, to the liquid plugs. The axis portions marked with the letters e and c correspond to the evaporator and condenser sections, respectively. (Reprinted from Ref. [108] with permission from Elsevier) 


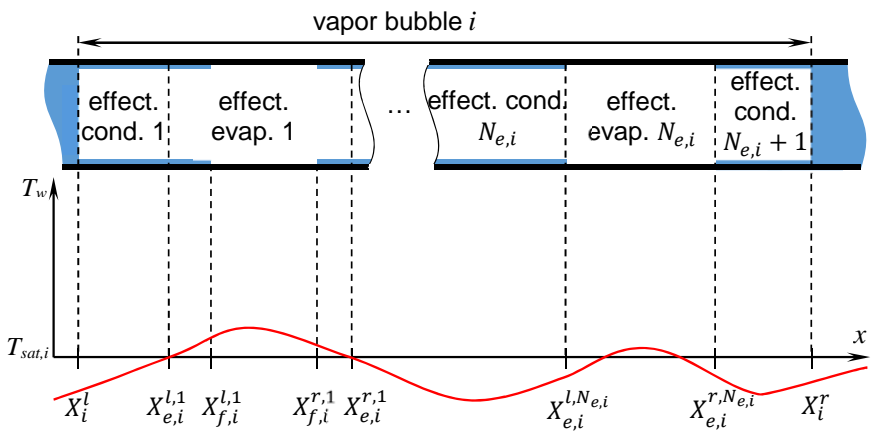

Figure 26: Geometry of the effective evaporators and condensers (top) corresponding to an example of the wall temperature distribution (bottom). Both liquid plug and liquid films are shown in blue. In this example, $X_{f, i}^{l, N_{e, i}}=X_{e, i}^{l, N_{e, i}}$ and $X_{f, i}^{r, N_{e, i}}=X_{e, i}^{r, N_{e, i}}$.

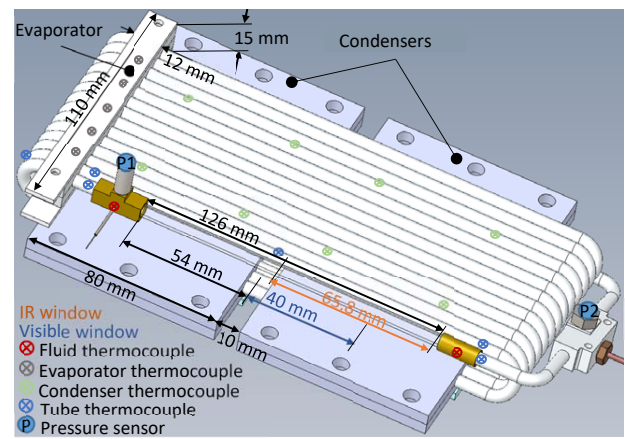

(a) PHP scheme. The transparent PHP branch is shown together with the position and length of its portion observed with the IR camera (IR window) and with a highspeed grayscale camera (Visible window).

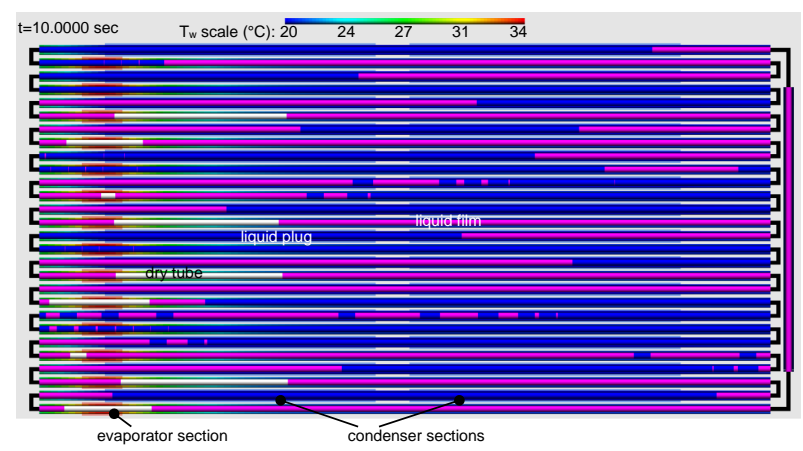

(b) The PHP representation in the CASCO software. The round turns are not pictured for simplicity; black lines just connect equivalent points which correspond to the extrema of each turn. The rose and blue rectangles around the tube (each second branch for this geometry) show the evaporator and condenser sections, respectively. For the fluid color code, see Fig. 19. The wall temperature is shown with a color scale pictured above PHP.

Figure 27: The large diameter PHP scheme and its CASCO representation [140].

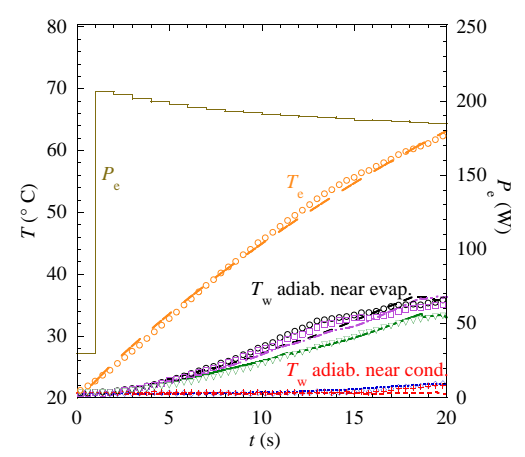

(a) Comparison of the temperature curves measured with the thermocouples shown in Fig. 27a and the averaged spreader temperature $T_{e}$. The injected power variation is also shown.

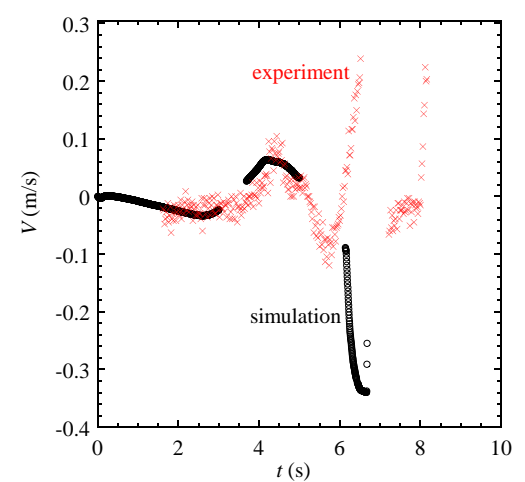

(b) Velocities of liquid plugs obtained from the IR image processing.

Figure 28: Comparison of the experiment and CASCO simulations for the large diameter PHP [140]. 